\title{
CARACTERIZACIÓN DE PARÁMETROS FISICOQUÍMICOS DE ESPECIES SUPRAMOLECULARES CON TENOXICAM
}

Tesis que presenta

Dafne Sarahia Guzmán Hernández

Para obtener el grado de

Doctora en Ciencias (Química)

Asesora: $\quad$ Dra. Ma. Teresa Ramírez Silva.

Jurado calificador:

Presidenta:

Secretario:

Vocal:

Vocal:

Vocal:

Dra. Annik Vivier Jégoux

Dr. Alberto Rojas Hernandez

Dr. Marcelo Galván Espinosa

Dra. Gabriela Roa Morales

Dra. Elena Páez Hernández

México, D.F. Mayo 2014

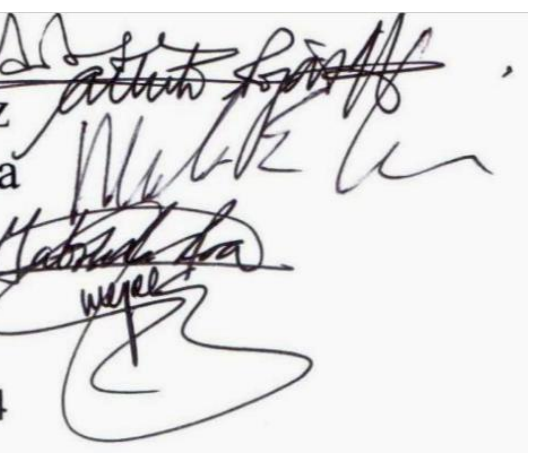


CARACTERIZACIÓN DE PARÁMETROS FISICOQUÍMICOS DE ESPECIES

\section{SUPRAMOLECULARES CON TENOXICAM.}


I. AGRADECIMIENTOS.-- 7

II. RESUMEN -

III. ABSTRACT- 10

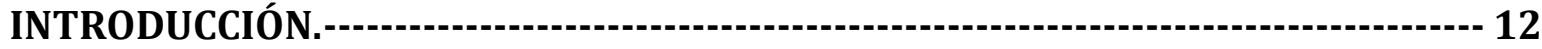

$i . \quad$ Estudios de caracterización de Tenoxicam.

ii. $\quad$ Formación de complejos supramoleculares conTenoxicam. -

IV. HIPÓTESIS.

V. OBJETIVO.-- 18

CAPÍTULO 1. ESTUDIO DEL TENOXICAM POR ESPECTROFOTOMETRÍA UV-VIS Y CÁLCULOS TEÓRICOS --:- 19

1.1 INTRODUCCIÓN. -

1.2 Estudio de estabilidad del Tenoxicam.

1.3 Las constantes de acidez de Tenoxicam.

1.4 Determinación de las constantes de acidez de Tenoxicam utilizando espectrofotometría UV-Vis. ---25

1.4.1 Reactivos.

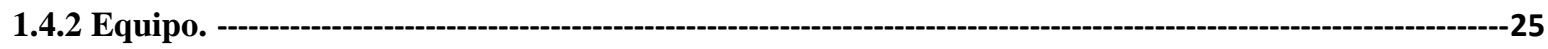

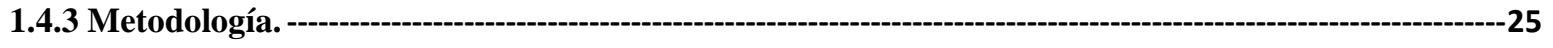

1.4.4 Constantes de acidez obtenidas mediante UV-Vis. -

1.5 Orden de desprotonación del Tenoxicam.-19

1.5.1 Cálculo del orden de desprotonación del Tenoxicam --

1.5.2 Detalles computacionales. -

1.5.3 Análisis de la desprotonación del $\mathrm{H}_{2} \mathrm{Tenox}^{+}$-

1.6 Conclusiones.---

CAPÍTULO 2. CARACTERIZACIÓN ELECTROQUÍMICA DEL TENOXICAM.---------- 33

2.1 Introducción. 
2.2 Estudio electroquímico del Tenoxciam. $--35$

2.2.1 Reactivos. $-35$

2.2.2 Equipo. $-35$

2.3.1 Respuesta del Tenoxicam en Voltamperometría Cíclica (VC).---------------------------------------------35

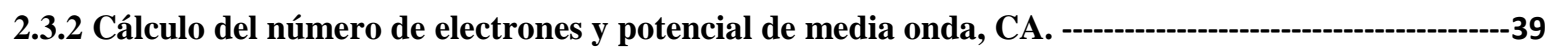

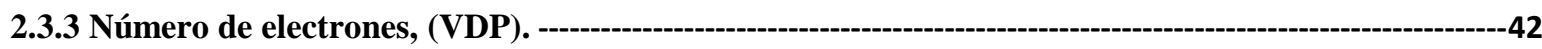

2.3.4 Respuesta de Tenoxicam en condiciones de convección forzada, voltamperometría lineal de disco rotatorio. (DR)

2.3.5 El coeficiente de difusión del Tenoxicam.

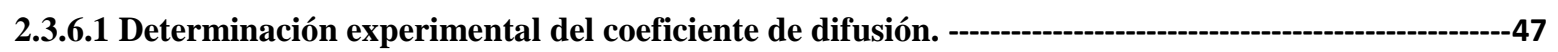

2.3.7 Determinación computacional del coeficiente de difusión.

2.3.5 Estudio electroquímico para las especies de HTenox (z) y Tenox-. ---------------------------------------48

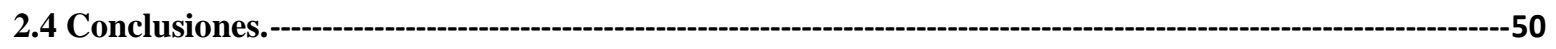

CAPÍTULO 3. PRODUCTOS DE OXIDACIÓN DE TENOXICAM. ---

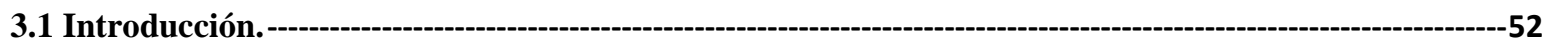

3.2 Metodología -----------------------------------------------------------------------------------------------------------------------'52

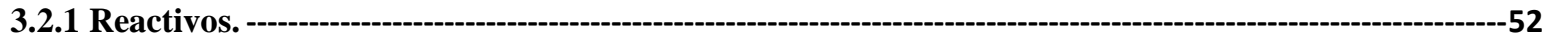

3.2.3 Equipo utilizado para los experimentos electroquímicos. ------------------------------------------------------52

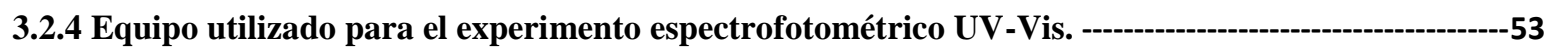

3.2.5 Equipo utilizado para el experimento de cromatografía líquida de alta resolución. ------------------53

3.2.6 Oxidación de la molécula de Tenoxicam. -

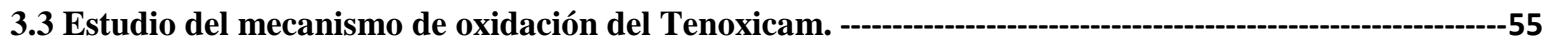

3.3.1 Detalles Computacionales para el estudio del mecanismo de oxidación. ----------------------55

3.3.2 Mecanismo de la oxidación de la molécula del Tenoxicam sin fragmentación.----------------56

3.3.3 Mecanismo propuesto de la oxidación de la molécula del Tenoxicam con fragmentación.-------------57

3.4 Estudio de la oxidación de la molécula de Tenoxicam mediante cromatografía líquida de alta

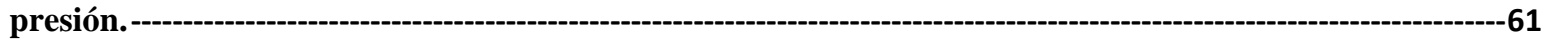

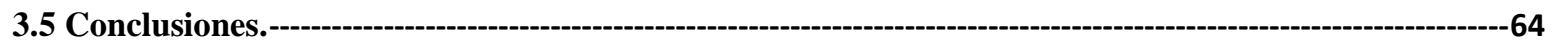

CAPÍTULO 4. FORMACIÓN DE COMPLEJOS SUPRAMOLECULARES.---:---:------- 65 


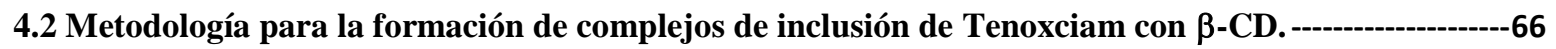

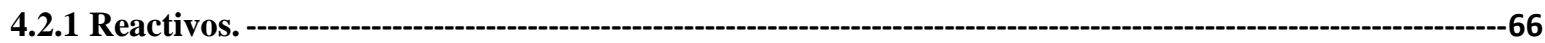

4.2.1.1 Equipo utilizado para los experimentos electroquímicos. ----------------------------------------------------66

4.2.1.2 Equipo utilizado para los experimentos espectrofotométricos UV-Vis. ------------------------66

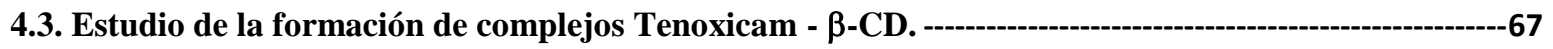

4.3.1 Determinación espectrofotométrica de la constante de inclusión del $\mathrm{H}_{2}$ Tenox ${ }^{+}$con $\beta-\mathrm{CD}$ a pH 0.410

4.3.2 Determinación espectrofotométrica de la constante de inclusión del HTenox(z) (pH 3.175) y Tenox

- (pH 7.150) con $\beta$-CD ---

4.3.3 Determinación electroquímica de la constante de inclusión del $\mathrm{H}_{2}$ Tenox $^{+}$con $\beta-\mathrm{CD}(\mathrm{pH}$ 0.420)-----79

4.3.4 Determinación electroquímica de la constante de inclusión del HTenox (pH 3.175) y Tenox ${ }^{-}(\mathrm{pH}$

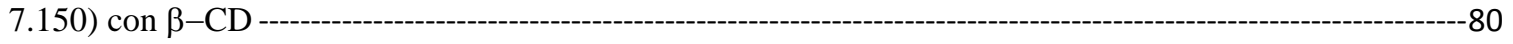

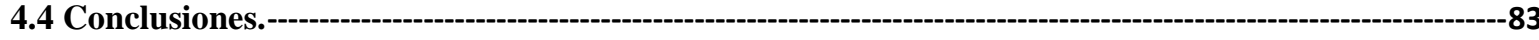

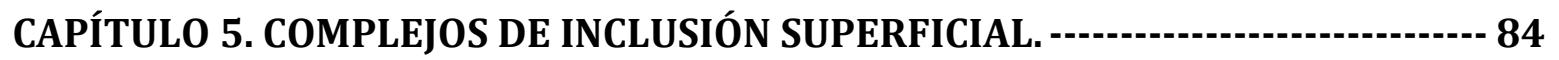

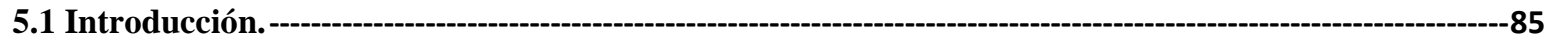

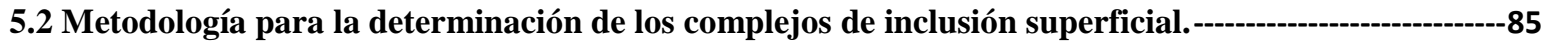

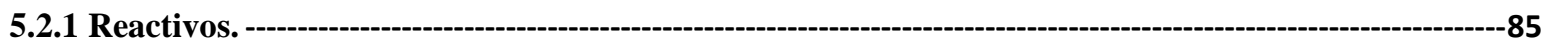

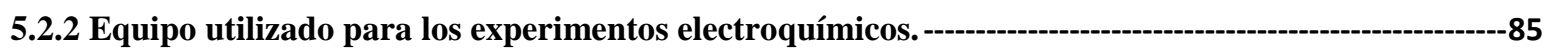

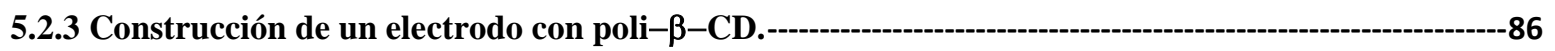

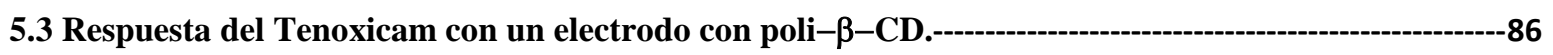

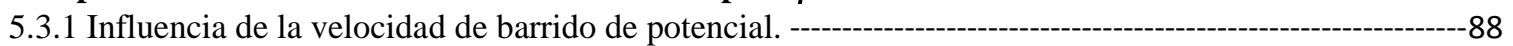

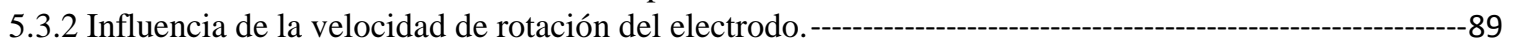

5.3.3 Influencia de la concentración de Tenoxicam. ---

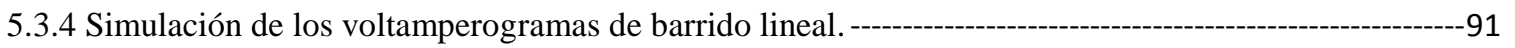

5.4 Determinación de las constantes de inclusión superficiales. -

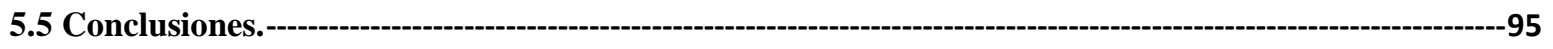

\section{CAPÍTULO 6. DETERMINACIÓN ANALÍTICA DE LA CONCENTRACIÓN DE}

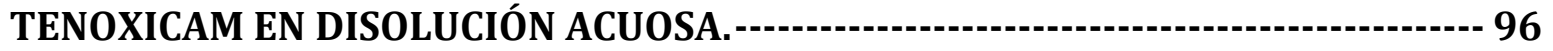

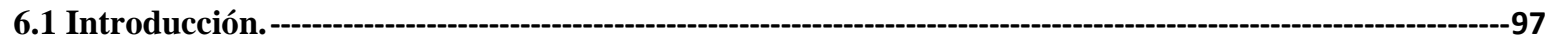

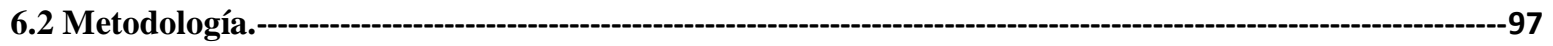

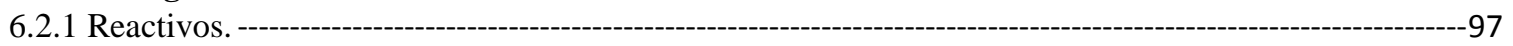

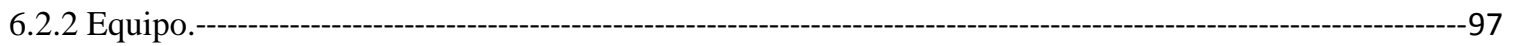

6.3 Cuantificación Tenox sobre un electrodo de pasta de carbón. ----------------------------------------------98 
6.3.2 Estudio de cuantificación por voltamperometría cíclica.

6.3.3 Estudio de cuantificación por voltamperometría diferencial de pulso. -------------------------------101

6.3.4 Estudio de cuantificación por voltamperometría cíclica para un electrodo de pasta de cabón

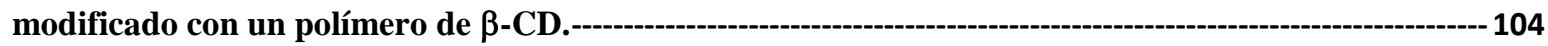

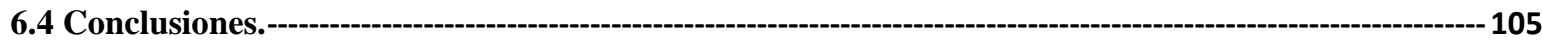

VI. CONCLUSIONES --:-- 106

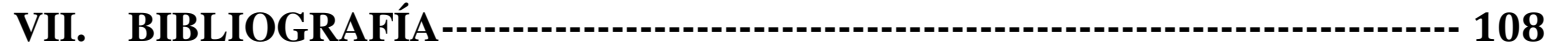

INTRODUCCIÓN. - --י-י--108

CAPÍTULO 1. -

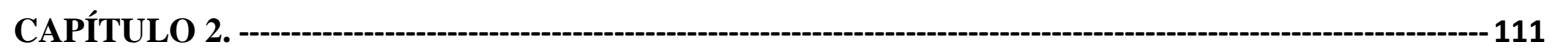

CAPÍTULO 3. ---

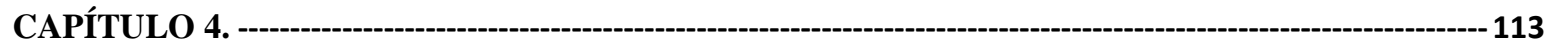

CAPÍTULO 5. - 114 


\section{AGRADECIMIENTOS.}

Al CONACyT, por la beca económica otorgada para la realización de los estudios de Posgrado. NÚMERO DE BECARIO (252083)

A la UAM-Iztapalapa, por darme la oportunidad de llevar a cabo mi formación de Posgrado.

Al Departamento de Química, Área de Química Analítica por brindarme un espacio para el desarrollo de este trabajo.

Al comité jurado: Dra. Annik Vivier Jégoux, Dr. Alberto Roja Hernández, Dr. Marcelo Galván Espinosa, Dra. Gabriela Roa Morales, Dra. Elena Paez Hernández. Por el tiempo que dedicaron en la revisión y por sus sugerencias para mejorar este trabajo de tesis.

Al Dr. Manuel Palomar Pardavé, por su amistad, apoyo y gran interés en mi desarrollo académico y profesional.

Al Dr. Mario Romero Romo, por sus valiosos consejos para poder expresarme correctamente en inglés académico, tanto de forma oral como escrita.

A la Dra. Silvia Corona Avendaño, por su apoyo, consejo y guía académica que me otorgo durante mi formación de doctorado.

A la Dra. Annia Galano, por su tiempo dedicado para fortalecer mis conocimientos y entendimiento el campo de la físico-química teórica.

Al Dr. Alberto Rojas Hernández, por su apoyo y seguimiento en el desarrollo de mi trabajo de investigación durante mi estancia en el doctorado.

A la Dra. Tere Ramírez, por toda su paciencia como asesora, maestra y sobre todo por ser una gran amiga.

A Fer, por su apoyo incondicional durante toda mi estadía en el posgrado.

A mis amigos Panchis, Anaid, Luis y Lupita por su compañía a lo largo del camino. 


\section{RESUMEN}

El trabajo aquí presentado se desarrolla sobre un anti inflamatorio no esteroidal, AINE, de la familia de los oxicams, denominado Tenoxicam, que es ampliamente utilizado para el tratamiento de enfermedades reumáticas y otros síntomas de dolor muscular e inflamación.

Se inició el estudio de Tenoxicam con su caracterización en solución acuosa. Se llevó a cabo un estudio de espectrofotometría de UV-Vis para el cálculo de las constantes de acidez (especiación química), teniendo como resultado un $\mathrm{pKa}_{1}=1.110 \pm 0.002$ y un $\mathrm{pKa}_{2}=5.270$ \pm 0.008. Se identificaron tres especies distintas según el $\mathrm{pH}$ de la solución: $\mathrm{H}_{2} \mathrm{Tenox}^{+}$, HTenox y Tenox`. Se encontraron dos posibles rutas para la primera desprotonación, donde se determinó, con ayuda de cálculos teóricos de DFT, que para esta primera desprotonación predomina la especie zwitteriónica.

Con el fin de proponer un mecanismo para la oxidación del Tenoxicam en todas sus formas, se llevó a cabo el estudio de la oxidación del medicamento en solución acuosa, utilizando métodos tanto teóricos como experimentales. Se encontró que la transferencia electrónica de la oxidación electroquímica para las tres especies está controlada por un proceso de difusión, y que la oxidación sigue un mecanismo electroquímico-químico. Las cinéticas química y electródica son rápidas y ocurren por la pérdida de dos electrones, que participan en la reacción.

Se encontró que el Tenoxicam presenta una reacción completamente irreversible debido a que presenta un mecanismo electroquímico-químico que genera productos no electroactivos. Debido a la importancia de conocer estos productos de oxidación, algunos investigadores han propuestos diversas moléculas que podrían corresponder a estos productos. Siguiendo esta misma línea, se llevó a cabo un estudio espectro-electroquímico para conocer la naturaleza más probable acerca de los productos de la oxidación y se apoyó este estudio con cálculos teóricos de DFT. Tras el análisis de dos esquemas de reacciones diferentes, que involucran hasta 10 moléculas y 12 caminos de reacción, se determinó que, en la oxidación electroquímica de Tenoxicam se producen mayoritariamente dos productos no electroactivos, que se forman por la fragmentación de la molécula cuando se pierden dos electrones. 
Se determinó el coeficiente de difusión, $\mathrm{D}=(4.396 \pm 0.058) 10^{-6} \mathrm{~cm}^{2} \mathrm{~s}^{-1}$, el cual se define muy bien con la corriente límite obtenida a partir de la curvas de voltamperometría lineal en condiciones hidrodinámicas y se describe adecuadamente con la ecuación de Levich. Por otra parte, a partir de la optimización del radio de la molécula del Tenoxicam utilizando un nivel de teoría M052X/6-31+g(d,p) y utilizando la aproximación de StokesEinstein, se estimó $\mathrm{D}=(4.54) 10^{-6} \mathrm{~cm}^{2} \mathrm{~s}^{-1}$, siendo este valor muy similar al valor experimental obtenido.

Por otro parte, debido a los efectos secundarios y su baja solubilidad que presenta el Tenoxicam, se han utilizado ciclodextrinas para tratar de disminuirlos estos efectos e incrementar la solubilidad, sin embargo son pocos los estudios que se pueden encontrar sobre la interacción del fármaco con las ciclodextrinas en solución acuosa. Por esta razón, una vez comprendido el comportamiento del Tenoxicam en solución acuosa, se llevó a cabo un estudio espectrofotométrico y electroquímico de su interacción con la $\beta$-ciclodextrina. En este estudio se determinaron las constantes termodinámicas de la formación del complejo de inclusión entre el Tenoxicam y la $\beta$-ciclodextrina en solución acuosa, y se observó que la formación del complejo de inclusión es posible no importando la naturaleza de las especies del Tenoxicam.

En el cuerpo humano el principio activo del medicamento es menor al 1\%, por lo que más del $99 \%$ del medicamento es desechado del cuerpo teniendo como resultado un problema ambiental en aguas residuales. Con la evidencia experimental acerca de la interacción del Tenoxicam con ciclodextrinas, en este trabajo se propone la utilización de un electrodo modificado con un polímero $\beta$-ciclodextrina con el fin de mejorar la cuantificación del medicamento en agua. Se llevó a cabo la caracterización del comportamiento del Tenoxicam con el electrodo modificado, se encontró que el proceso presentado sobre el electrodo modificado con $\beta$-ciclodextrina es de adsorción. Se determinó la concentración superficial, $\Gamma \mathrm{r}$, con un valor máximo de $1.51 \times 10^{-10} \mathrm{~mol} \mathrm{~cm} \mathrm{~cm}^{-2}$ para una concentración añadida en la solución de Tenox de $64 \mathrm{mM}$ y finalmente se determinaron las constantes de inclusión superficial en solución acuosa a partir de la formación de un polímero de $\beta$-ciclodextrina. El electrodo modificado permite detectar el Tenoxicam en agua, mediante voltamperometría cíclica, con una mejora en los límites de detección (0.86 $\pm 0.28)$ y cuantificación $(4.93 \pm 0.69)$. 


\section{ABSTRACT}

The present research work has been developed on a non steroidal anti inflammatory, NSAI, that belongs to the oxicams family, namely the Tenoxicam, which is widely used in general for the treatment of rheumatic diseases and other muscle pain and inflammation symptoms.

Since it has had widespread use as anti inflammatory and there is not a deeper study on its characterization, an UV-Vis spectrophotometric study was carried out for calculating its acidity constants, which were pKa1 $=1.110 \pm 0.002$ and $\mathrm{pKa} 2=5.270 \pm 0.008$. Also, the predominant three different ionic species according to the Tenox solution $\mathrm{pH}$ were: $\mathrm{H}_{2}$ Tenox $^{+}$, Tenox and HTenox ${ }^{-}$. In this study of chemical speciation, two possible routes for the first deprotonation were studied, which were determined using theoretical DFT calculations, resulting that for this first deprotonation, the neutral zwitterionic species predominates, $\operatorname{HTenox}(\mathrm{z})$.

Subsequently, various studies on the oxidation of the Tenox molecule in aqueous solution were conducted, where it was found that the electrochemical oxidation for the three Tenox species was controlled by a mass transfer process, taking place through an electrochemical - chemical mechanism, where the electrode and chemical kinetics were fast, exchanging two electrons, $n=2$, involved in the electrochemical reaction. Further, the diffusion coefficient, $\mathrm{D}=(4.396 \hat{\mathrm{A}} \pm 0.058) 10^{-6} \mathrm{~cm} 2 \mathrm{~s}^{-1}$ was determined, which appeared very well defined with the limiting current obtained from the linear voltammetry curves under hydrodynamic conditions, also, well described through the Levich equation. Moreover, from the optimization of Tenox molecule radius using theory level M052X/6$31+\mathrm{g}(\mathrm{d}, \mathrm{p})$ and the Stokes- Einstein approximation, it was estimated as $\mathrm{D}=(4.396 \hat{\mathrm{A}} \pm$ $0.058) 10^{-6} \mathrm{~cm} 2^{-1}$, being similar to the experimental value.

Considering that the Tenox presents a completely irreversible reaction due to an electrochemical-chemical mechanism, which generates no electroactive products, therefore, there was the impending necessity to establish the nature of these oxidation products; hence, spectro-electrochemical studies were carried out to widen the possibility to assess the nature of the oxidation products. The results obtained showed that, after the analysis of two different reaction schemes involving up to 10 molecules and 12 reaction paths, the electrochemical oxidation of Tenox is produced principally from two non-electroactive 
products, which are mainly formed by the fragmentation of the molecule when two electrons are lost.

Once this was understood, the Tenox behavior in aqueous solution was studied through spectrophotometry and an electrochemical study of the interaction of $\beta$ Cyclodextrin with Tenox, where the thermodynamic formation constants of the inclusion complex were determined, i.e., the formation of the inclusion complex is possible regardless of the nature of Tenox species.

Also, the constants of a surface inclusion complex in aqueous solution after formation of a $\beta$-cyclodextrin polymer on the surface of a carbon paste electrode was determined, showing that an adsorption process was taking place in the presence of the $\beta$ cyclodextrin-modified electrode, so that the surface concentration, $\Gamma \mathrm{r}$, reached a maximum value of $1.51 \times 10-10 \mathrm{~mol} \mathrm{~cm}-2$ of the Tenox concentration added in a solution of $64 \mathrm{mM}$.

Finally, a study of the analytical determination of Tenox was presented, where an improvement in the quantification and determination limits and sensibility for the Tenox molecule were obtained when a $\beta$-cyclodextrin-modified electrode was used. 


\section{INTRODUCCIÓN.}

El Tenoxicam (Tenox), Fig. I.1 es un fármaco anti inflamatorio no esteroidal del grupo de los oxicams el cual es indicado para el tratamiento del dolor leve o moderado, en especial en condiciones como la artritis reumatoide, osteoartritis y otras enfermedades reumáticas, así como en el tratamiento sintomático del lumbago, gota aguda, miositis, bursitis, tendonitis y esguinces ${ }^{[\mathrm{I} .1]}$. Como analgésico y antiinflamatorio es tan eficaz y potente como el piroxicam, el diclofenaco o la indometacina, más no presenta propiedades como antitérmico ${ }^{[1.2]}$

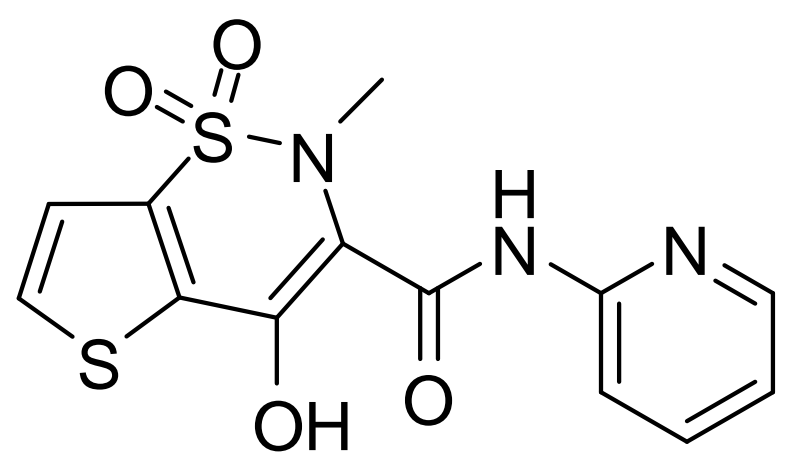

Figura I.1. Molécula de Tenox.

Estos fármacos del grupo de anti inflamatorios no esteroidales son un grupo heterogéneo de estructuras hidrofóbicas con grupos funcionales hidrofílicos, los cuales llegan a presentar baja estabilidad y solubilidad, de aquí el interés por el estudio de estos fármacos. Los estudios clínicos y el control de calidad de fármacos necesitan técnicas de análisis precisas, sensibles y selectivas, que permitan la detección y cuantificación de estos fármacos en muestras biológicas y farmacéuticas de una manera confiable. Así mismo, se requiere cuantificar el Tenox y sus metabolitos en aguas para el control de la contaminación ambiental.

Una manera de aumentar la solubilidad de moléculas orgánicas, como lo es la del Tenox, en medio acuoso es formando especies supramoleculares, las cuales resultan de la asociación de dos o más especies moleculares, presentando, de manera específica, un reconocimiento molecular mediante interacciones débiles de tipo no covalente ${ }^{[\mathrm{I.3}]}$. 
Las Ciclodextrinas (Fig. I.2) permiten la formación de complejos de inclusión, debido a que estas moléculas son oligosacáridos cíclicos con un número de moléculas de glucosa de 6, 7 y 8, correspondiendo $\alpha, \beta$ y $\gamma$ Ciclodextrina. Tienen una estructura espacial que es troncocónica con una cavidad interior que permite la incorporación de un sustrato [I.4].

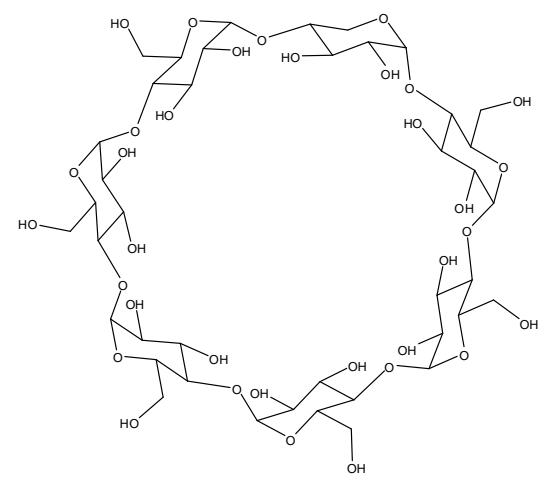

Figura I.2. Estructura espacial de la $\beta$-Ciclodextrinas con 7 moléculas de glucosa.

La configuración molecular tridimensional más estable para estas moléculas es de forma de cono truncado con una apertura grande y otra pequeña, que presenta grupos hidroxilos primarios y secundarios hacia la parte que está en contacto con el disolvente. Estos grupos le confieren mayor solubilidad a estas moléculas en medio acuoso.

Las características precisas de las Ciclodextrinas, hace a estas moléculas excelentes para la formación de sistemas supramoleculares, en específico con la molécula de Tenox.

Para la investigación de este tipo de fármacos, una herramienta utilizada en los últimos años son los métodos teóricos, los cuales ayudan a entender o explicar los comportamientos observados experimentalmente; estos métodos dentro de la investigación sobre fármacos permiten establecer posibles estructuras y actividades de diversos grupos de moléculas. Así mismo estos métodos como herramienta, ayudan a describir estructuras electrónicas de las moléculas, la naturaleza del enlace químico, a interpretar y predecir propiedades geométricas, así como también a describir la energía de ionización, la afinidad electrónica, la energía de interacción, entre otras características estructurales.

Los métodos utilizados en la mecánica cuántica tienen la característica de utilizar solamente constantes atómicas fundamentales (método ab initio) o emplean datos experimentales al momento de establecer parámetros en parte de los cálculos (método 
empírico). Otro método empleado en los cálculos es la teoría de funcionales de la densidad (DFT), el cual lleva como ventaja sobre los otros métodos un ahorro en cálculos computacionales. Los métodos anteriormente mencionados son parte de la química teórica que incluye métodos matemáticos y algoritmos computacionales combinados con las leyes fundamentales de la física para describir sistemas químicos, los cuales influyen en la química molecular ${ }^{[1.5]}$.

\section{i. $\quad$ Estudios de caracterización de Tenoxicam.}

Ya que el Tenox se utiliza en medio acuoso, es importante conocer sus características en este medio, por lo que algunos autores han empezado a publicar estudios que permiten tener acceso a información química como son sus constantes de acidez en agua mediante electroforesis capilar y resonancia magnética nuclear, donde determinan que el Tenox presenta dos protones ácidos en medio acuoso a los cuales se le asocian los valores encontrados, $\mathrm{pKa}_{1}=1.074 \pm 0.025 \mathrm{y} \mathrm{pKa}_{2}=5.142 \pm 0.027^{[\mathrm{I} .6]}$. Teniendo como resultado tres especies distintas para el Tenox.

Así mismo se sabe que el Tenox actúa reduciendo el dolor y la inflamación producidas por células fagocíticas, especialmente neutrófilos y eosinófilos matan a las bacterias invasoras a través de la llamada "explosión respiratoria" [I.7]. Durante este fenómeno se produce un aumento dramático en la oxidación metabólica. La enzima NADPH oxidasa es responsable de iniciar esta explosión catalizando la reducción monovalente de un oxígeno molecular a un anión radical superóxido $\left(\mathrm{O}^{2-}\right)$, que se convierte rápidamente en peróxido de hidrógeno $\left(\mathrm{H}_{2} \mathrm{O}_{2}\right)$. En presencia de $\mathrm{H}_{2} \mathrm{O}_{2}$ y un factor oxidable (por ejemplo, $\mathrm{Cl}^{-}, \mathrm{Br}^{-}$), peroxidasas de leucocitos tales como la mieloperoxidasa y peroxidasa de eosinófilos dan lugar a productos oxidantes tales como el ácido hipocloroso (HC1O), que ejercen una alta actividad bactericida ${ }^{[1.8]}$. Contrariamente a este papel fisiológicamente importante y útil, muy a menudo la mayoría de los productos oxidantes oxidan inevitablemente diversas biomoléculas y causan citotoxicidad debido a la alta reactividad de estos productos.

Durante la última década muchos investigadores han postulado que la inflamación es promovida por la citotoxicidad de productos oxidantes; en un sitio de la inflamación, estos productos (especialmente $\mathrm{HClO}$ ) inactivan un inhibidor de $\alpha$-proteinasa, permitiendo 
la actividad no controlada de la proteasa ${ }^{[\mathrm{I.9}, \mathrm{I.10]}}$. No obstante, pocos trabajos han prestado atención del mecanismo de supresión de los AINES ${ }^{[\text {[.12-I.14] }}$. Tanto en Fenilbutazona ${ }^{[\text {[.12] y }}$ en Tenox ${ }^{[\mathrm{I} .13]}$ se encontró que reaccionan fácilmente con especies de oxígeno activo (incluyendo $\mathrm{HC1O}$ ) en un átomo del carbono de la $\beta$-dicetona y para actuar como un eliminador de estas especies. Ichihara y colaboradores ${ }^{[\text {I.13, I.15] }}$ han demostrado que un extracto de leucocitos, que tenía una alta actividad de la peroxidasa (en su mayoría mioloperoxidasa), convierte al Tenox en cuatro metabolitos nuevos en presencia de $\mathrm{H}_{2} \mathrm{O}_{2}$ los cuales se conocen como 4,5-dihidro-4-oxo-5-metiliminopirido (1,2 a) imidazol, ácido 2carboxilo, ácido 2-carboxil-3-thlofenesuftonico, y N-metil-N'-(2 - piridil) oxamida. Además, también encontraron ${ }^{[1.13]}$ que la adición de un anión haluro tal como $\mathrm{Cl}^{-}$se mezcla con el plomo y la reacción de esto da la formación de tres metabolitos. Ichihara et al ${ }^{\text {[I.13, I.15] }}$ afirman que estos metabolitos fueron probablemente formados por una reacción de oxidación de un electrón en el átomo de carbono central de la fracción $\beta$-dicetona de Tenox. El Tenox es un cofactor para la reducción de peroxidasas y esta capacidad puede explicar al menos una parte del efecto anti-inflamatorio del Tenox.

Dentro de los pocos estudios con métodos electroquímicos ${ }^{\text {[I.16- I.17] }}$, se encuentra el de Ozaltin et al. ${ }^{\left[{ }^{[18]}\right.}$ quien reporta la determinación y caracterización del Tenox mediante una determinación polarográfica por voltamperometría diferencial del pulso en fármacos y en sangre, utilizando un electrodo de gota de mercurio. El-Maali y Hassan ${ }^{\text {[I.17] }}$ 1levaron a cabo una investigación sobre el comportamiento acerca de la oxidación de algunos anti inflamatorios, piroxicam y Tenox sobre un electrodo de pasta de carbón (EPC) por voltamperometría cíclica, encontrando que estos compuestos muestran un comportamiento similar. Otros autores han encontrado que la oxidación electroquímica de otros AINEs del grupo de los oxicams meloxicam ${ }^{[\mathrm{I} .19]}$, lornoxicam ${ }^{[\mathrm{I} .19]}$ y piroxicam ${ }^{[\mathrm{I} .19-\mathrm{I} .21]}$, son irreversibles.

A pesar de los estudios que se han realizado hasta ahora, no se ha llevado a cabo una caracterización electroquímica más profunda de Tenox que podría considerar, por ejemplo, la cantidad de electrones que se pierden durante la oxidación del Tenox, así como el valor del coeficiente de difusión en medio acuoso. Además, teniendo en cuenta la irreversibilidad electroquímica observada para oxicams, la oxidación no se ha examinado a fondo. 


\section{ii. $\quad$ Formación de complejos supramoleculares conTenoxicam.}

Al ser el Tenox es un fármaco de amplio uso se han desarrollado investigaciones acerca de los efectos secundarios que puede presentar y en ellos se ha encontrado que el Tenox no muestra efectos secundarios mutagénicos o carcinogénicos en pruebas de animales, sin embargo aproximadarmente el $12.5 \%$ de los pacientes muestran efectos secundarios o alteraciones en las pruebas de laboratorio. Algunos de estos síntomas se manifiestan muy ligera y temporalmente. El tratamiento ha sido detenido solo en un $1 \%$ de los pacientes. Los efectos secundarios más frecuentes son los efectos gastrointestinales, acidez estomacal, náuseas, diarrea y estreñimiento, efectos sobre el sistema nervioso central como son efectos dérmicos, irritación prurito, manchas, fotodermatosis en la piel y efectos renales ${ }^{[1.22]}$. Para aumentar la solubilidad de Tenox en medio acuoso y disminuir sus efectos secundarios se ha propuesto el uso de ciclodextrinas (CDs), en particular de $\gamma$-CD [I.23].

Mediante microscopia electrónica de barrido, estudios termogavimetricos, calorimetría diferencial y experimentos de espectroscopia infra roja, algunos autores han mostrado que la formación de complejos de inclusión en fase sólida entre Tenox y $\alpha, \beta$ y $\gamma$ Ciclodextrina es posible ${ }^{[1.22, \text { I.25] }}$

No obstante, pese a la importancia de este sistema, el cálculo de la constante de inclusión entre Tenox y ciclodextrinas en medio acuoso ha sido poco estudiado. Hasta donde se sabe el único trabajo al respecto es el reportado por Banerjee et al. ${ }^{[1.26]}$, quienes por medio de fluorescencia y medidas de la anisotropía de fluorescencia han reportado que la constante de enlace de Tenox con $\beta$-ciclodextrina tiene el valor de $115 \pm 13 \mathrm{M}^{-1}$ en una mezcla etanol-agua. Más aún, indican que la encapsulación ocurre solamente para la forma neutra del Tenox $(1.1<\mathrm{pH}<5.2)$.

Tomando en consideración todo lo antes descrito, en este trabajo se lleva a cabo un estudio de caracterización más profunda acerca del Tenox, apoyándose en métodos de espectrofotometría de UV-Vis, cromatorgrafía HPLC, estudios teóricos DFT y técnicas electroquímicas. Así mismo debido a las características de los sistemas supramoleculares, en este trabajo se plantea el desarrollo experimental para la caracterización y determinación de la interacción del Tenox con la $\beta$-Ciclodextrina para formar especies supramoleculares 
con el fin de proporcionar nuevas perspectivas en relación al Tenox en solución acuosa y de esta manera poder obtener parámetros fisicoquímicos importantes en medio acuoso.

\section{HIPÓTESIS.}

El caracterizar el comportamiento químico de la molécula de Tenoxicam y sus complejos supramoleculares en solución acuosa permitirá desarrollar métodos de análisis cuantitativos con los que se obtendrán mejoras en los parámetros analíticos.

\section{OBJETIVO.}

Caracterizar fisicoquímicamente la molécula de Tenoxicam en solución acuosa y determinar la formación de complejos supramoleculares con la molécula de Tenoxicam.

Para cumplir con el objetivo general es necesario efectuar los siguientes objetivos particulares.

$\checkmark$ Analizar los espectros de comportamiento a diferentes valores de $\mathrm{pH}$ del Tenox.

$\checkmark$ Determinar las constantes de acidez del Tenox.

$\checkmark$ Optimizar la geometría para la molécula de Tenox mediante cálculos computacionales.

$\checkmark$ Obtener los espectros teóricos de UV-Vis a partir de la optimización de la molécula del Tenox.

$\checkmark$ Analizar el comportamiento electroquímico para diferentes valores de $\mathrm{pH}$ del Tenox.

$\checkmark$ Evaluar la interacción de Tenox con la $\beta$-Ciclodextrina.

$\checkmark$ Analizar el comportamiento espectrofotométrico y electroquímico para diferentes valores de $\mathrm{pH}$ de la interacción del Tenox con la $\beta$-Ciclodextrina. 


\section{CAPÍTULO 1. TENOXICAM.}

\subsection{INTRODUCCIÓN.}

Una de las caracterizaciones fisicoquímicas de mayor importancia es el cálculo de las constantes de acidez ya que éstas pueden ser un parámetro para el entendimiento del fenómeno de cuantificación química, la velocidad de reacción, la formación de las sales, la formación de complejos y el transporte iónico, así las propiedades acido base influyen en la toxicidad, y las propiedades farmacéuticas de los compuestos orgánicos, la actividad biológica o el transporte iónico, este parámetro ayuda a entender la adsorción, distribución

y transporte de un medicamento ${ }^{[1.1]}$, es por ello que en este capítulo se presenta el estudio de estabilidad y determinación de constantes de acidez para la molécula de Tenox.

\subsection{Estudio de estabilidad del Tenoxicam.}

Con base a lo reportado en la literatura se ha encontrado que el Tenox es inestable cuando es expuesto a la luz solar, en estas condiciones la degradación del Tenox presenta una banda en 460nm, con el fin de analizar la estabilidad de dicho compuesto para nuestro sistema en medio acuoso se lleva a cabo un estudio UV-Vis, donde se sigue el comportamiento del Tenox a $25^{\circ} \mathrm{C}$, en presencia de oxígeno y con la exposición a la luz, los espectros obtenidos en función del tiempo para las especies en medio acido, neutro y básico se muestran en la figura 1.1 . 
Figura 1.1. Espectros de absorción UV-Vis obtenidos a diferentes tiempos de estar en exposición a la luz, en presencia de oxígeno y a temperatura de $25^{\circ} \mathrm{C}$. Condicione de trabajo: Tenox 7.5x10-6 $\mathrm{M} \mathrm{pH} 1.00$ (a), pH 7.42 (b) y pH 11.45 (c). 
A partir de los espectros obtenidos es posible observar la banda característica de la especie a pH 1.00 del Tenox ubicada en 357nm (Figura 1.1a), ésta se mantiene sin cambios al menos en un periodo de 60min (figura2a inserto), por lo que el Tenox en estas condiciones es estable, para la especie obtenida a $\mathrm{pH}=7.42$ y 11.46 se obtuvieron los espectros y se observa que no hay cambios significativos en su comportamiento espectral, por lo que se puede concluir que las especies del Tenox a $\mathrm{pH}$ acido, básico y neutro son estables.

Sin embargo al corroborar la degradación del Tenox por la exposición directa a la luz solar medio acuoso a $\mathrm{pH} 8.74$ (figura1.2), los resultados reportados en la literatura revelan que el espectro del Tenox cambia en función del tiempo, más en la región espectral, solo se observa la banda tipica del Tenox y aun después de 60min de la exposición a la luz del sol, no se presenta ninguna banda en 460nm, lo que indica que no existe la formación de productos de fotodegradacion del Tenox en el sistema de trabajo.

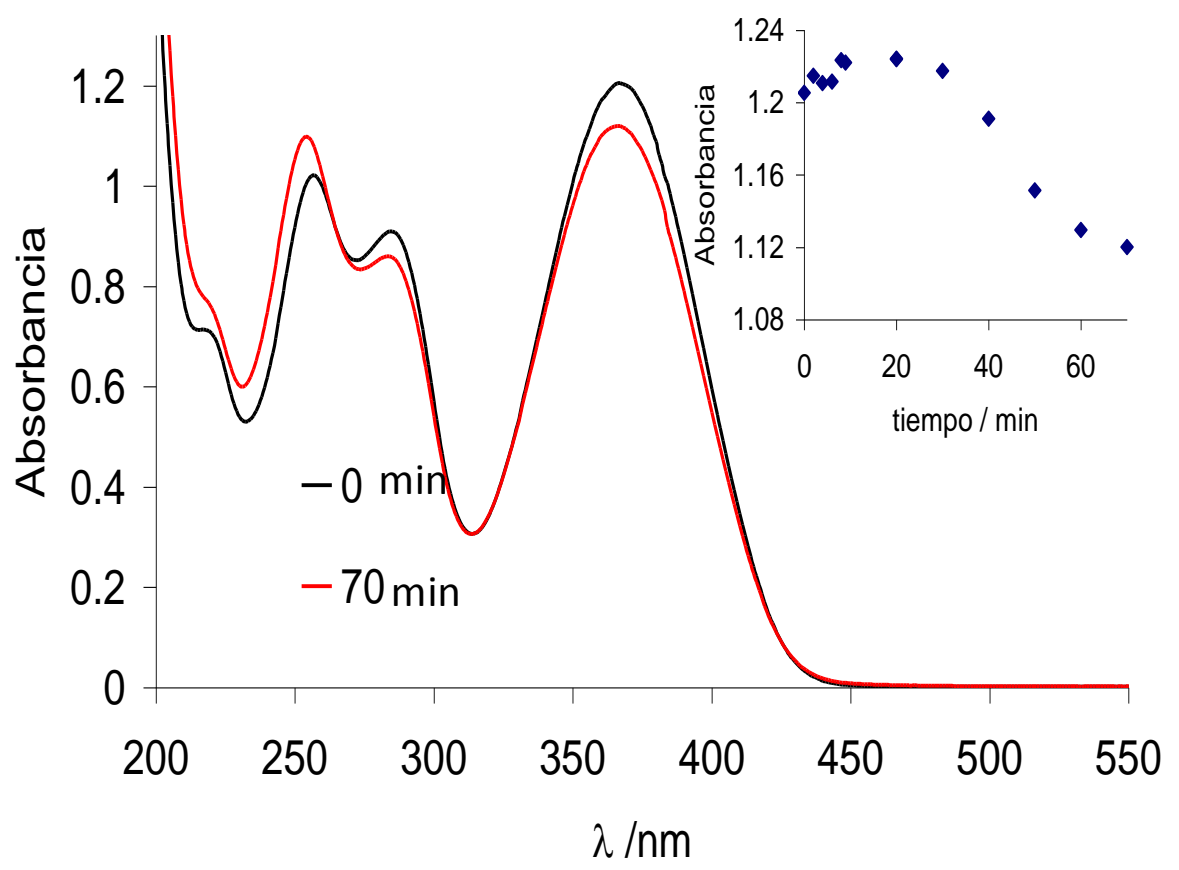

Figura 1.2. Espectros de absorción del Tenox $7.5 \times 10^{-6} \mathrm{M} \mathrm{pH}$ b) 8.74 tomado en diferentes tiempos de haber estado expuestos a $25^{\circ} \mathrm{C}$, en presencia de oxígeno y con la exposición de la luz solar. En el inserto se presenta el gráfico del máximo de absorción en función del tiempo. 
Bajo estas circunstancias, donde el Tenox mostró ser estable a diferentes valores de pH y bajo condiciones ambientales, se desarrolló el método para la determinación de sus constantes de acidez en medio acuoso.

1.3 Las constantes de acidez de Tenoxicam.

La molécula de Tenox presenta dos protones ácidos (fig. 1.3) por lo que es posible determinar sus constantes de acidez.

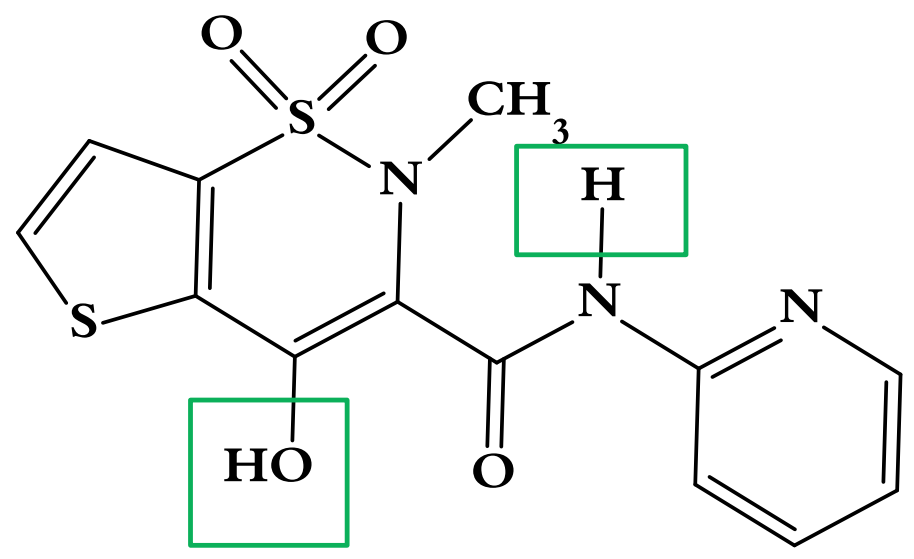

Figura 1.3. Protones ácidos de molécula de Tenox

Para determinar las constantes de acidez de una molécula se pueden utilizar diferentes técnicas experimentales. Una de ella es electroforesis capilar de zona (ECZ), esta técnica está basada en la movilidad de partículas cargadas bajo la influencia de un campo eléctrico. Esta técnica en particular realiza una separación en función de la carga de las especies.

Las constantes de acidez de Tenox fueron calculadas mediante esta técnica por Rodríguez-Barrientos y colaboradores ${ }^{[1.2]}$, obteniendo dos valores de pKa para los equilibrios mostrados en la tabla 1.1. 
Tabla 1.1 Constantes de acidez de Tenox obtenidas mediante la técnica experimental de $\mathrm{ECZ}$ a $25^{\circ} \mathrm{C}$ y fuerza iónica $0.1 \mathrm{M}^{[1.2]}$.

\begin{tabular}{|c|c|}
\hline Equilibrio & $\mathrm{pKa} \pm \sigma$ \\
\hline$H_{2}$ Tenox $^{+} \rightleftharpoons$ HTenox $+H$ & $1.075 \pm 0.025$ \\
\hline HTenox $\rightleftharpoons$ Tenox $^{-}+H$ & $5.142 \pm 0.027$ \\
\hline
\end{tabular}

Rodríguez-Barrientos en este mismo trabajo determinó las constantes de acidez de Tenox por medio de resonancia magnética nuclear (RMN), esta técnica se basa en los desplazamientos químicos de las especies. Con esta técnica obtuvo las constantes presentadas en la tabla 1.2.

Tabla 1.2 Constantes de acidez de Tenox obtenidas mediante la técnica experimental de RMN en DMSO ${ }^{[1.2]}$.

\begin{tabular}{|c|c|c|}
\hline \multicolumn{2}{|c|}{ Equilibrio } & $\mathrm{pKa} \pm \sigma$ \\
\hline $\mathrm{H}_{2}$ Tenox & HTenox $+H$ & $1.923 \pm 0.151$ \\
\hline HTenox & Tenox $^{-}+H$ & $4.422 \pm 0.069$ \\
\hline
\end{tabular}

Otra técnica experimental empleada para determinar constantes es la espectrofotometría de ultra violeta visible (UV-Vis). Debido a que por medio de esta técnica se llevará a cabo el estudio de la interacción de las distintas especies de Tenox con $\beta-C D$, se realiza la determinación de las constantes de acidez para calibrar el método, además de que se trabaja con una fuerza iónica mucho mayor $(0.2 \mathrm{M})$ de la reportada por Rodriguez-Barrientos, et. al. con el fin de alcanzar valores de pH’s más ácidos. 
1.4 Determinación de las constantes de acidez de Tenoxicam utilizando espectrofotometría UV-Vis.

\subsubsection{Reactivos.}

La solución de Tenox fue preparada a partir del reactivo grado A R Merck. Para ajustar el pH se utilizó $\mathrm{NaOH}$ (Merck 99\%) y $\mathrm{HCl} /($ Merck 37\%). Todas las soluciones fueron preparadas usando agua desionizada tipo $1(18.2 \mathrm{M} \Omega \mathrm{cm})$ libre de materia orgánica, obtenida de US Filter PURE- LAB Plus UV la cual fue burbujeada con nitrógeno comprimido (Praxair) $(1 \mathrm{~min} / \mathrm{mL})$.

\subsubsection{Equipo.}

Los espectros de absorción fueron obtenidos mediante un espectrofotómetro Lambda 20 Perkin Elmer.

El pH de las soluciones fue medido usando un potenciómetro Mettler Lab pHM240 con una resolución de $0.001 \mathrm{de} \mathrm{pH}$ con un electrodo combinado de vidrio de intervalo de pH $\quad 0-14$ ((Mettler Toledo InLab® Expert NTC30 + DIN/RCA $)$ ). Durante la experimentación se controló la temperatura a $25^{\circ} \mathrm{C}$ y se mantuvo la solución en atmósfera inerte de nitrógeno.

\subsubsection{Metodología.}

La estimación de las constantes de acidez pKa se realizó partiendo de una solución de Tenox $7.5 \times 10^{-5} \mathrm{M}$ en $\mathrm{NaOH} 0.01 \mathrm{M}$ y realizando adiciones de $\mathrm{HCl} 0.1 \mathrm{M}$ y $\mathrm{NaCl} 0.1 \mathrm{M}$. Para cada adición se tomó la lectura de $\mathrm{pH}$ y de potencial, además del espectro de absorción.

La información obtenida se alimentó al programa computacional SQUAD ${ }^{[1.3]}$, para el cálculo de las constantes espectrofotométricas.

\subsubsection{Constantes de acidez obtenidas mediante UV-Vis.}

En la Figura 1.4, se muestran los espectros de absorción de Tenox en el intervalo de pH 0.46 a 13.44, en donde es posible observar un máximo de absorción en 354 nm para valores de $\mathrm{pH}$ ácidos en el intervalo de 0.4 y 1 , el cual presenta un efecto batocrómico y un efecto hipocrómico conforme se incrementa el pH así mismo se aprecia la formación de una banda de absorción para longitudes de onda menores a 300nm. A partir de pH 6 el pico 
máximo de absorción presenta un efecto hipsocrómico y un efecto hipocrómico, y se aprecia la formación de dos bandas para longitudes de onda menores a 300nm.

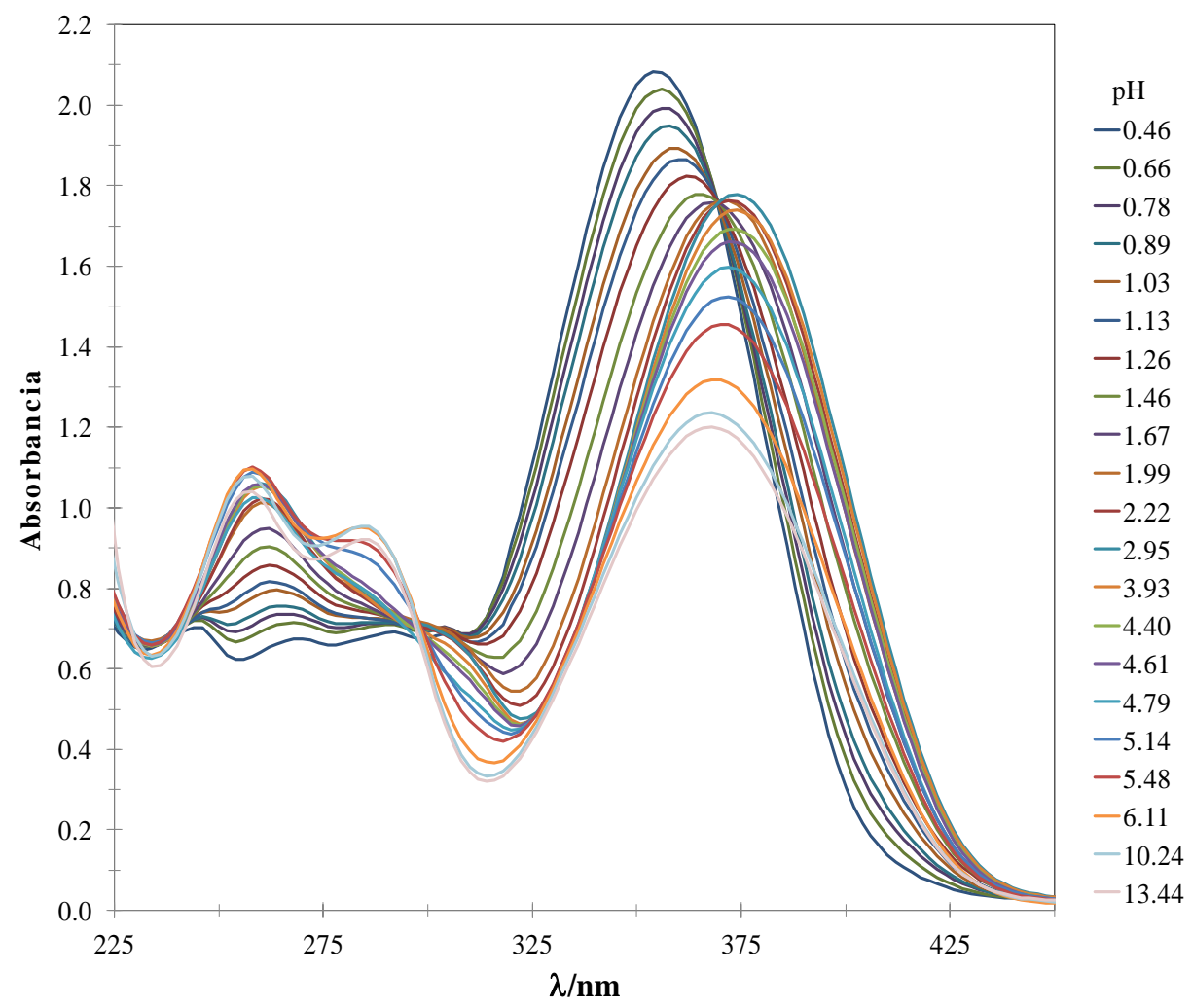

Figura 1.4 Espectros de absorción a diferentes $\mathrm{pH}$ para Tenox $7.5 \times 10^{-6} \mathrm{M}$ obtenidos a partir de diferentes adiciones de $\mathrm{NaOH}$.

El cálculo de las constantes de acidez para el Tenox se obtuvo mediante el programa computacional SQUAD. Este programa se alimentó con 70 espectros de absorción experimentales en un intervalo de longitud de onda $220 \mathrm{~nm}$ a $500 \mathrm{~nm}$, los valores de $\mathrm{pH}$ obtenidos para cada espectro de absorción y la concentración del analito (Tenox), además de una propuesta del modelo químico.

A partir de SQUAD se obtienen los valores de las constantes de acidez para cada equilibrio asociado, estas se presentan en la tabla 1.3. 
Tabla 1.3. Constante de acidez obtenida a partir de los espectros mostrados en la Figura 1.2 y el programa SQUAD para 70 espectros de absorción y un intervalo de longitud de onda $220 \mathrm{~nm}$ a $500 \mathrm{~nm}$.

\begin{tabular}{|c|c|c|}
\hline & Equilibrio & $\log \beta \pm 0$ \\
\hline $\mathrm{H}_{2}$ Tenox $^{+}$ & $\rightleftharpoons$ HTenox $+H$ & $6.385 \pm 0.008$ \\
\hline HTenox & $\rightleftharpoons$ Tenox $^{-}+H$ & $5.277 \pm 0.006$ \\
\hline
\end{tabular}

$\mathrm{DS}=0.0204 \mathrm{U}=3.9 \times 10^{-4}$

En la figura 1.5 se presenta los coeficientes de absortividad molar, $\varepsilon$, obtenidos a partir de SQUAD para las distintas especies de Tenox.

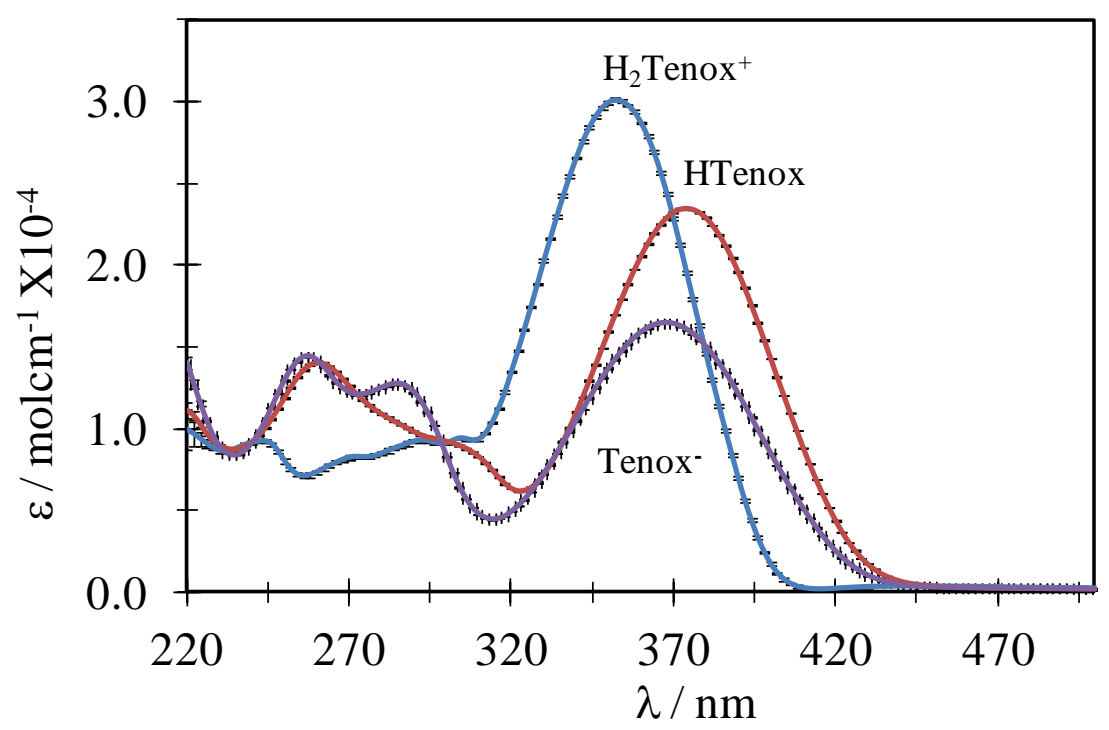

Figura 1.5. Coeficientes de absorción generados a partir de SQUAD para las distintas especies de Tenox a partir de los espectros de absorción de la figura 1.4.

Una vez refinadas las constantes de acidez de Tenox y con los coeficientes de absortividad obtenidos, se realizó el ajuste de los espectros de absorción de Tenox y se comparan con los experimentales. La Figura 1.5 muestra los espectros simulados para los pH más representativos. 


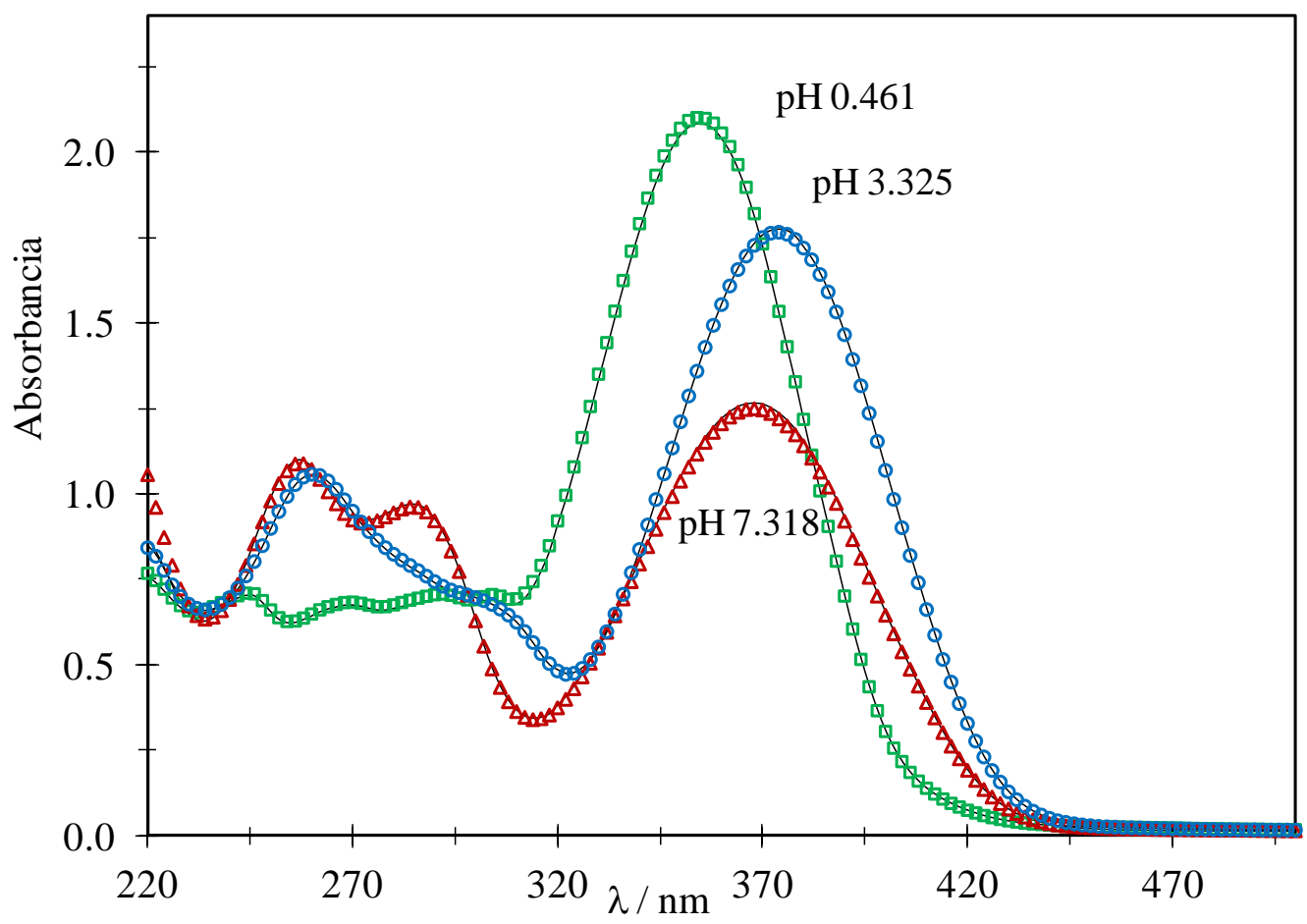

Figura 1.6 Espectros UV-Vis Tenox $75 \mu \mathrm{M}$ ajustados a partir de los datos calculados (línea) y comparados con los experimentales (marcador).

Como se puede observar en la figura 1.6 los espectros ajustados concuerdan con los obtenidos experimentalmente. Este resultado indica que los datos ajustados obtenidos representan el comportamiento experimental, por lo que las constantes de acidez calculadas son confiables.

Así entonces se ha caracterizado mediante espectrofotometría UV-Vis el Tenox donde se tienen el predomino de tres especies para la molécula de Tenox, como se muestra en el diagrama de zonas de predominio lineal de la figura 1.7, sin embargo para la especie neutra se tienen dos posibles desprotonaciones, de las cuales puede predominar la especie neutra o la especie zwiteriónica. 

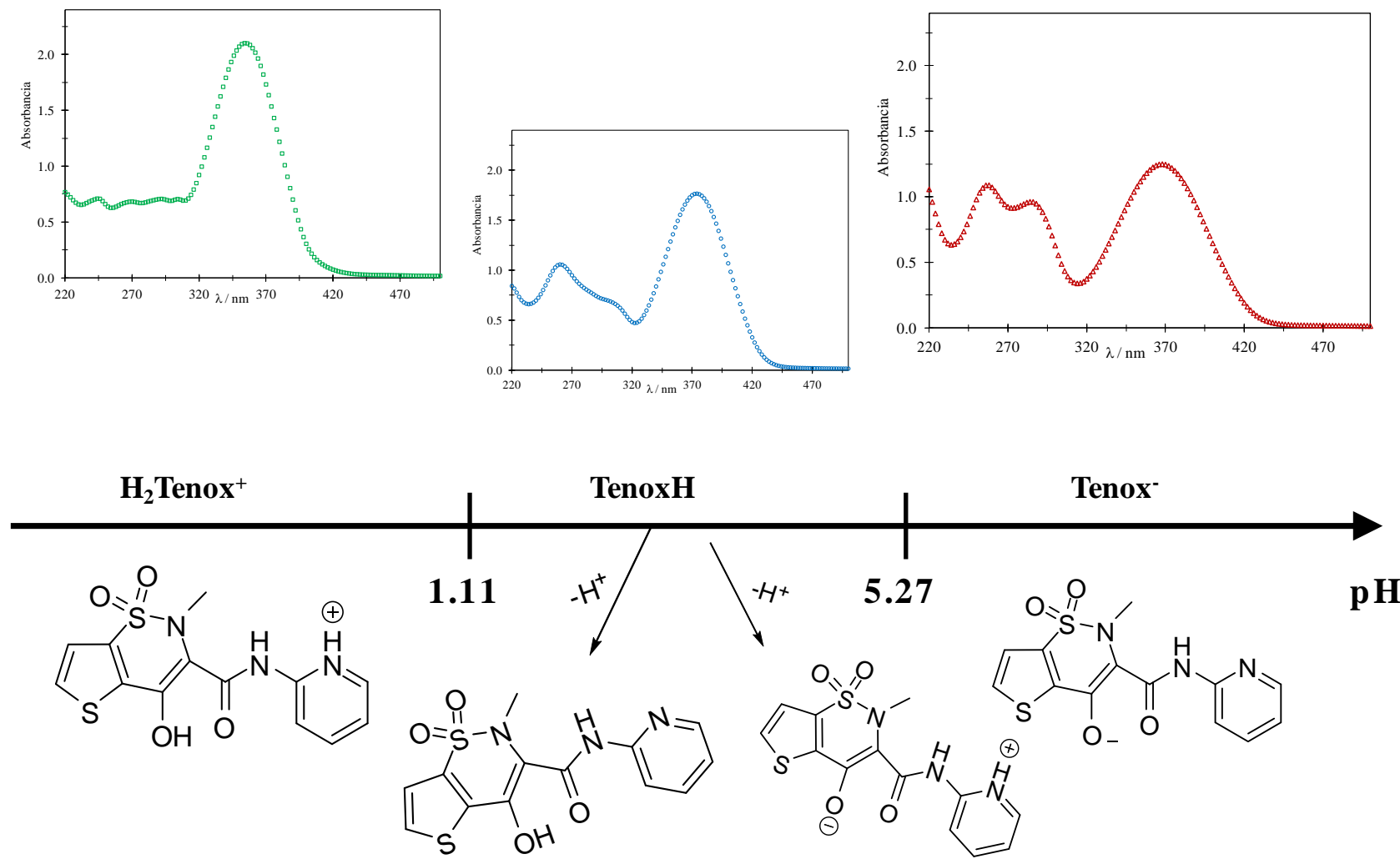

Figura 1.7 Diagrama lineal de zonas de predominio en función de $\mathrm{pH}$ para la molécula de Tenox.

1.5 Orden de desprotonación del Tenoxicam.

Para discernir el predominio entre la especie zwiteriónica y la especie neutra del Tenox, se realizaron cálculos teóricos utilizando la teoría de funcionales de la densidad (DFT por sus siglas en inglés).

\subsubsection{Cálculo del orden de desprotonación del Tenoxicam}

\subsubsection{Detalles computacionales.}

Los cálculos de optimización de geometría y frecuencia se llevaron a cabo utilizando el funcional M05-2X ${ }^{[1.4]}$ y el conjunto base 6-3 $1+\mathrm{G}(\mathrm{d}, \mathrm{p})$. Los cálculos de optimización de geometría y frecuencia fueron realizados en solución, utilizando el modelo continuo SMD ${ }^{[1.5]}$ y agua como solvente. 
Todos los cálculos se realizaron con el paquete de programas Gaussian $09^{[1.6]}$. Las correcciones termodinámicas a $298 \mathrm{~K}$ fueron incluidas en el cálculo de energías relativas. Los espectros calculados se han calculado utilizando la teoría de funcionales de la densidad dependiente el tiempo (TD-DFT), con transición vertical que implica hasta tres estados excitados.

\subsubsection{Análisis de la desprotonación del $\mathrm{H}_{2}$ Tenox $^{+}$}

Debido a la estructura química de Tenox y el hecho de que tiene dos valores de pKa, su proceso de desprotonación en solución acuosa puede implicar diferentes especies químicas. Por ejemplo, la primera etapa de desprotonación, de la molécula de Tenox totalmente protonada $\left(\mathrm{H}_{2}\right.$ Tenox ${ }^{+}$) para el neutro (HTenox), puede dar lugar a especies de zwiterion o en una especie neutra. Dado que la interacción de un producto de este tipo con su entorno será muy diferente dependiendo de su naturaleza, es muy importante tener un conocimiento exacto de cuál especie química de Tenox resulta después de la etapa de desprotonación.

Dos rutas de desprotonación han sido investigadas para la especie $\mathrm{H}_{2} \mathrm{Tenox}^{+}$(ver esquema 1.I): una ruta conduce a la especie neutra (HTenox) y la otra conduce al zwitterion $(\operatorname{HTenox}(z))$. En el cálculo de energías, como se puede observar en el esquema 1.I, se encontró que la especie zwitterionica es significativamente la más bajo en energía. Por lo tanto, se prevé que sea la que se forme durante la primera desprotonación de $\mathrm{H}_{2} \mathrm{Tenox}^{+}$.

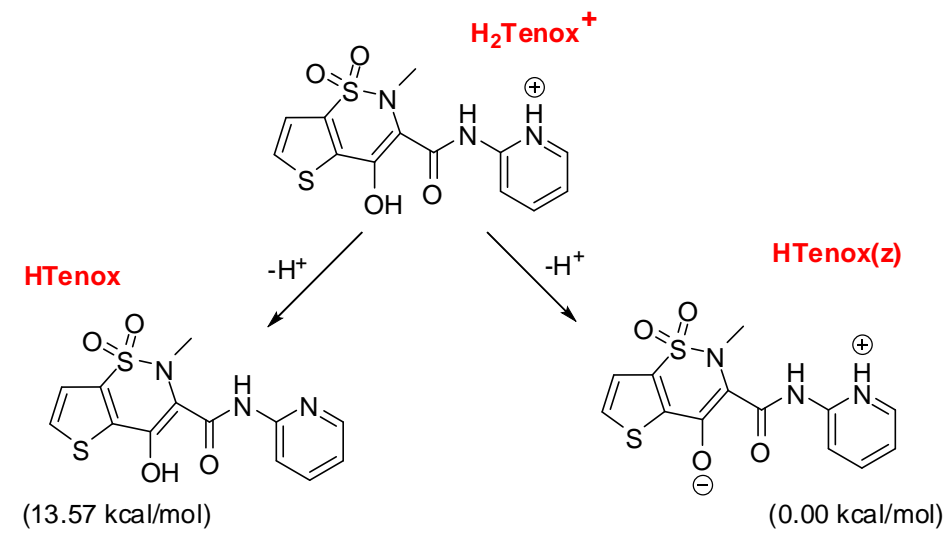

Esquema 1.I Ruta de la primera desprotonación de la especie $\mathrm{H}_{2} \mathrm{Tenox}^{+}$. 
En la figura 1.8(a) se pueden observar los espectros UV-Vis para las especies de HTenox, HTenox(z) y $\mathrm{H}_{2} \mathrm{Tenox}^{+}$calculados teóricamente encontrándose que las formas de todos ellos son muy similares. Presentan dos máximos de absorción en la región de 310 a $340 \mathrm{~nm}$ y un segundo máximo de absorción alrededor de $260 \mathrm{~nm}$. La banda principal en el espectro de UV de HTenox(z) es desplazada al rojo por $20 \mathrm{~nm}$ con respecto al espectro de UV de $\mathrm{H}_{2}$ Tenox ${ }^{+}$, mientras que es azul desplazado por $8 \mathrm{~nm}$ en el espectro de UV de HTenox. En la figura 1.8 (b) se puede observar la comparación de estos espectros con el experimental correspondiente a pH 3.325 donde se puede concluir que la desprotonación de $\mathrm{H}_{2} \mathrm{Tenox}^{+}$corresponde a la especie $\operatorname{HTenox}(\mathrm{z})$. Además de que la energía y los criterios espectrales, apoyan que la especie HTenox que predomina corresponde a las especie zwitterionica.
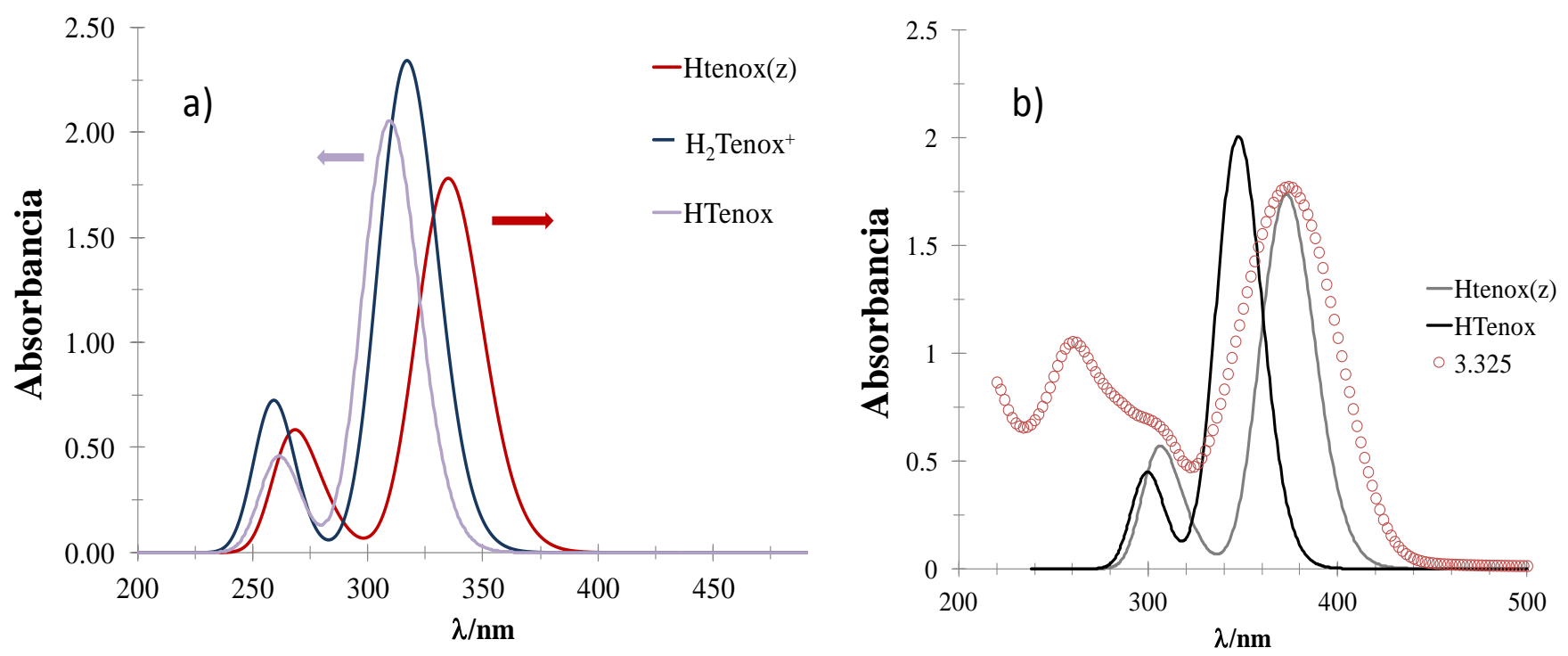

Figura 1.8. (a) Espectros UV-Vis calculados para las especies HTenox, HTenox(z) y $\mathrm{H}_{2}$ Tenox $^{+}$. (b) Comparación de los espectros UV-Vis de HTenox(z) y HTenox con el espectro experimental obtenido a $\mathrm{pH} 3.325$.

\subsection{Conclusiones.}

Se obtuvieron las constantes de acidez de la molécula de Tenox, y se presentan las especies que predominan junto con su caracterización espectrofotométrica experimental y teórica DFT. Ver figura 1.9. 


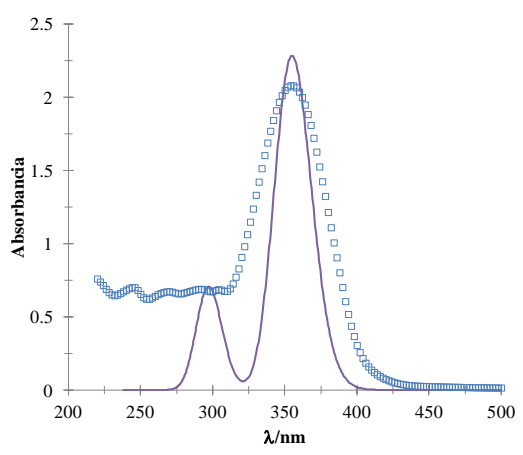

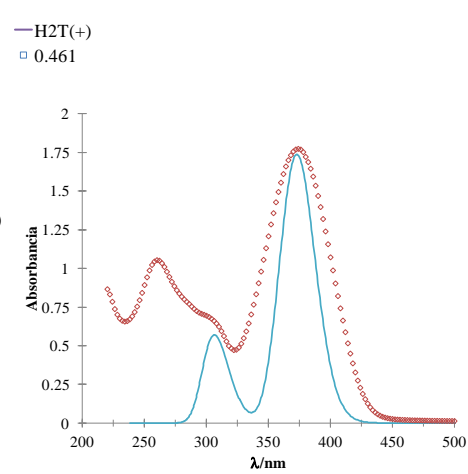

$\operatorname{TenoxH}(z)$

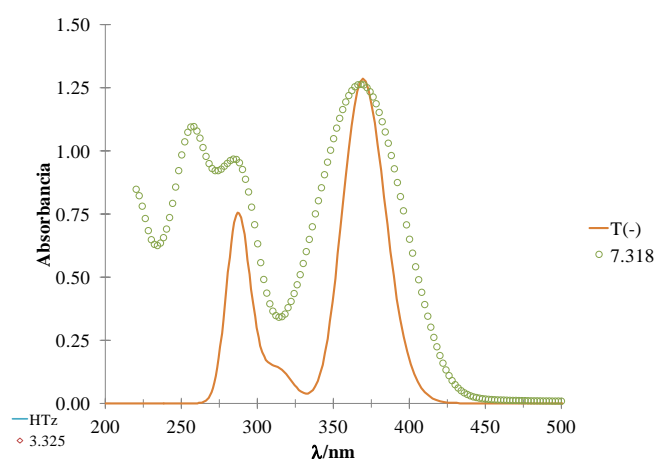

$\mathbf{H}_{2}$ Tenox $^{+}$

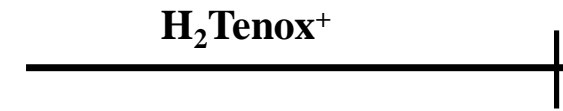

1.110

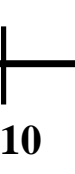<smiles>CN1C(C(=O)Nc2ccccn2)=C(O)c2sccc2S1(=O)=O</smiles><smiles>CN1C(C(=O)Nc2ccccn2)=C(O)c2sccc2S1=O</smiles>

5.270

Figura 1.9. Diagrama lineal de zonas de predominio en función de $\mathrm{pH}$ para la molécula de Tenox. Caracterización espectrofotométrica experimental (marcadores) y DFT (línea continua).

Así mismo se determinó el camino de desprotonación a seguir a partir de la especie $\mathrm{H}_{2} \mathrm{Tenox}^{+}$, siendo la especie predominante el zwitterion la cual denominaremos en los siguientes capítulos como HTenox(z).

Cabe resaltar que el método calibrado para obtener las constantes de acidez mediante espectroscopia de UV-Vis, son similares a las obtenidas por Rodriguez-Barrientos et. al ${ }^{[1.2]}$ por medio de electroforesis capilar y RMN (Ver tabla 1.4).

Tabla 1.4. Comparación de las constantes de acidez obtenidas a partir de espectrofotometría UV-Vis comparadas con las reportadas.

\begin{tabular}{|c|c|c|c|c|}
\hline \multirow{2}{*}{\multicolumn{2}{|c|}{ Equilibrio }} & \multicolumn{3}{|c|}{$\mathrm{pKa} \pm \sigma$} \\
\hline & & UV-Vis* & $\mathrm{CZE}^{[1.2]}$ & $\mathrm{NMR}^{[1.2]}$ \\
\hline $\mathrm{H}_{2}$ Tenox $^{+}$ & $\rightleftharpoons$ HTenox $+H$ & $1.110 \pm 0.002$ & $1.074 \pm 0.025$ & $1.923 \pm 0.151$ \\
\hline HTenox & $\rightleftharpoons$ Tenox $^{-}+H$ & $5.270 \pm 0.008$ & $5.142 \pm 0.027$ & $4.422 \pm 0.069$ \\
\hline
\end{tabular}

*Obtenidos en este trabajo. 

ELECTROQUÍMICA DEL TENOXICAM. 


\subsection{Introducción.}

En la figura 2.1 es posible observar que la molécula de Tenox presenta grupos que podrían oxidarse perdiendo cierto número de electrones, por lo que una vez caracterizada la molécula de Tenox mediante espectrofotometría UV-Vis y determinado el orden de desprotonación, es importante su caracterización electroquímica para el desarrollo de métodos electroquímicos para la cuantificación de Tenox.

Así, en este capítulo se estudia el comportamiento electroquímico de Tenox en solución acuosa apoyado en distintas técnicas electroquímicas como son voltamperometría cíclica (VC), voltamperomería diferencial de pulso (VDP), cronoamperometría (CA) y electrodo de disco rotatorio (DR). A pesar de que este estudio se llevó a cabo para las diferentes especies de Tenox, para efectos prácticos se presenta detalladamente el estudio para la especie protonoda $\mathrm{H}_{2} \mathrm{Tenox}^{+}$, presentando al final los resultados obtenidos para las otras dos especies, HTenox (z) y Tenox'.

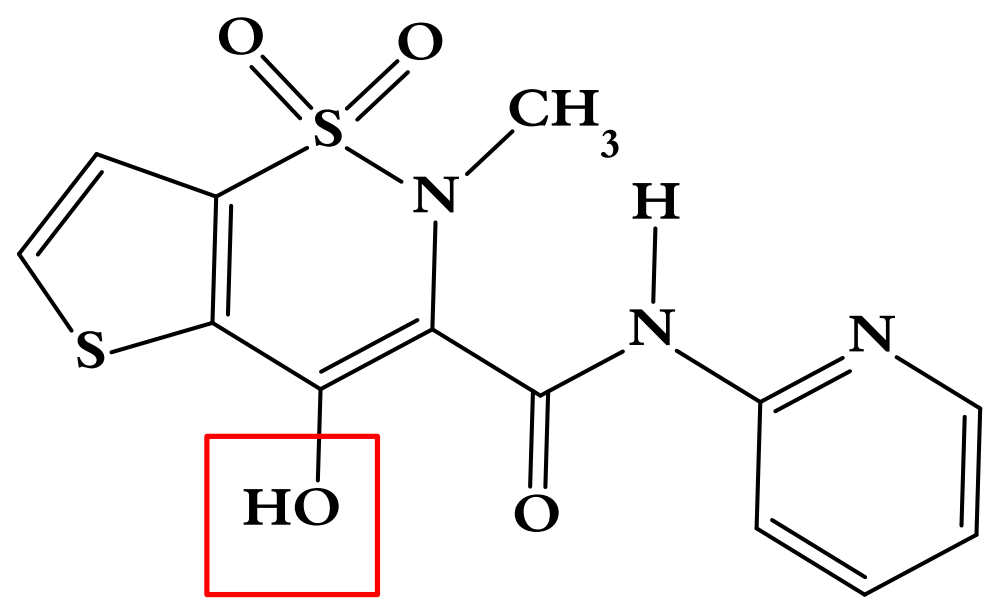

Figura 2.1. Probables sitios de oxidación de la molécula de Tenox 
2.2 Estudio electroquímico del Tenoxciam.

\subsubsection{Reactivos.}

Todos los reactivos son de grado analítico, Tenox (FLUKA), hidróxido de sodio (JT Baker) y ácido clorhídrico (Monterrey), todas las soluciones fueron preparadas con agua desionizada.

\subsubsection{Equipo.}

Para los experimentos electroquímicos de VC, VDP y CA se utilizó una celda convencional de tres electrodos; como electrodo de trabajo se utilizó un electrodo de pasta de carbón (EPC) el cual fue preparado a partir de polvo de Grafito (Johnson Matthey $1 \mu \mathrm{m}$, $99.9 \%)^{[2.1,2.2]}$. Como contra-electrodo se utilizó un alambre de platino (BAS MW-1032) y como electrodo de referencia se utilizó un electrodo $\mathrm{Ag} / \mathrm{AgCl}$ saturado (BAS MF-2052). Los experimentos electroquímicos se llevaron a cabo con un potenciostato (EPSILONBass-i).

Para el experimento electroquímico en condiciones de convección forzadas (DR) se utilizó un EPC (3 mm de diámetro) acoplado a un controlador de electrodo de disco rotatorio (RDE-BAS-2) como electrodo de trabajo.

El pH de las soluciones fue medido usando un potenciómetro Mettler Lab pHM240 con una resolución de 0.001 de $\mathrm{pH}$ con un electrodo combinado de vidrio de intervalo de pH 0-14 ((Mettler Toledo InLab® Expert NTC30 + DIN/RCA)). Durante la experimentación se controló la temperatura a $25^{\circ} \mathrm{C}$ y se mantuvo la solución en atmósfera inerte de nitrógeno.

\subsubsection{Respuesta del Tenoxicam en Voltamperometría Cíclica (VC).}

En la Figura 2.2 se muestran el VC obtenido en el sistema EPC / $12 \mu \mathrm{M}$ Tenox, $0.2 \mathrm{M} \mathrm{Cl}^{-}$a $\mathrm{pH} 0.405$ donde predomina la especie $\mathrm{H}_{2}$ Tenox ${ }^{+}$. El barrido de potencial se inicia en potencial de circuito abierto $\left(\mathrm{E}_{\mathrm{i}}=0\right)$ hacia potenciales positivos a una velocidad de barrido de potencial de $0.1 \mathrm{Vs}^{-1}$ y es posible apreciar que se presenta un pico de oxidación en $0.78 \mathrm{~V}$. Al invertir el barrido de potencial se espera observar el pico de reducción correspondiente al pico anódico presente, sin embargo no se presenta pico de reducción alguno. 


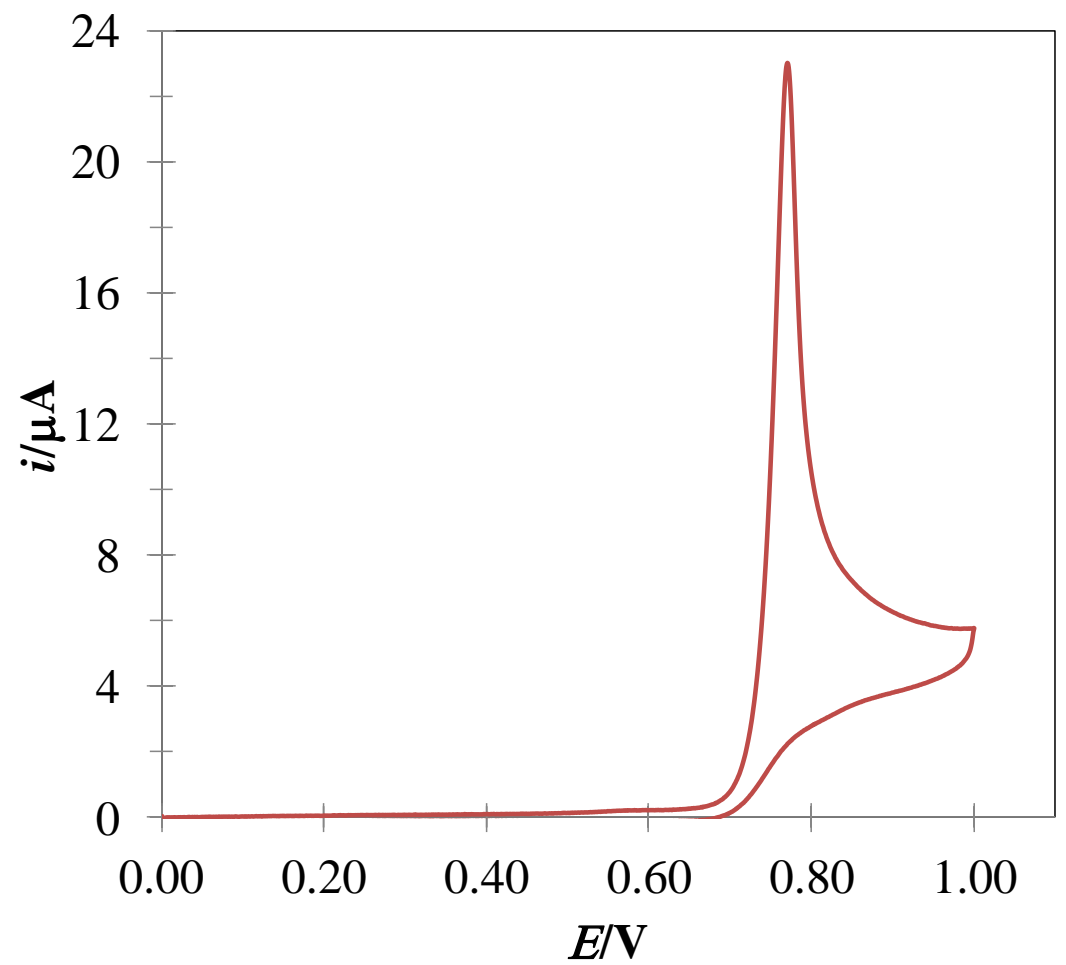

Figura 2.2. Voltamperograma cíclico obtenidos en el sistema EPC / $12 \mu \mathrm{M}$ Tenox, $0.2 \mathrm{M} \mathrm{Cl}^{-}$a pH 0.405. El barrido de potencial se inicia en $0 \mathrm{~V}$ en dirección anódica a una velocidad de barrido fue de $0.1 \mathrm{Vs}^{-1}$

$\mathrm{Al}$ obtener solo el pico de oxidación en el VC es importante saber qué limita la transferencia de carga. Para ello se lleva a cabo un estudio de VC para diferentes velocidades de barrido de potencial.

En la figura 2.3 se presenta una familia de VC's a diferentes velocidades de barrido de potencial para la especie $\mathrm{H}_{2} \mathrm{Tenox}^{+}$(pH 0.405), las cuales van desde $20 \mathrm{mVs}^{-1}$ hasta 200 $\mathrm{mVs}^{-1}$ 


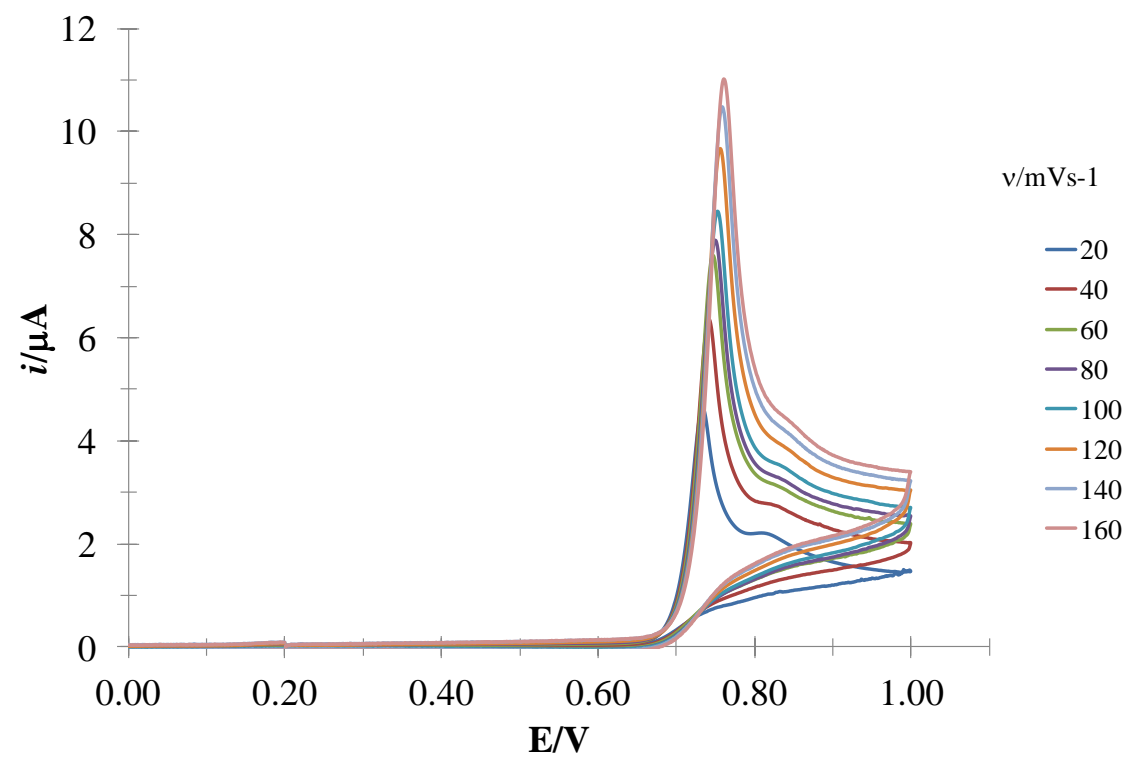

Figura 2.3. Familia de VC's a diferentes velocidades de barrido de potencial mostradas en la figura para el sistema EPC/ Tenox $7 \mu \mathrm{M}$ a $\mathrm{pH} 0.410$.

La expresión de Randles-Sevcik (ecuación 2.1) ${ }^{[2.3]}$, indica que la transferencia de carga está limitada por la difusión cuando se presenta una función lineal de la corriente de pico en función de la raíz cuadrada de la velocidad de barrido de potencial.

$$
i_{p a}=\left(2.688 \times 10^{5}\right) n^{3 / 2} S D_{R}^{1 / 2} C_{R} v^{0.5}
$$

Donde $i_{p a}$ es la corriente de pico anódica, $n$ el número de electrones, $S$ el área del electrodo, $D_{R}$ el coeficiente de difusión, $C_{R}$ la concentración del analito y $v$ la velocidad de barrido de potencial.

Así entonces, en la figura 2.4 se presenta el gráfico de $i_{p}=f\left(v^{0.5}\right)$, donde se puede observar una tendencia lineal lo cual indica que la transferencia de carga está controlada por la difusión. 


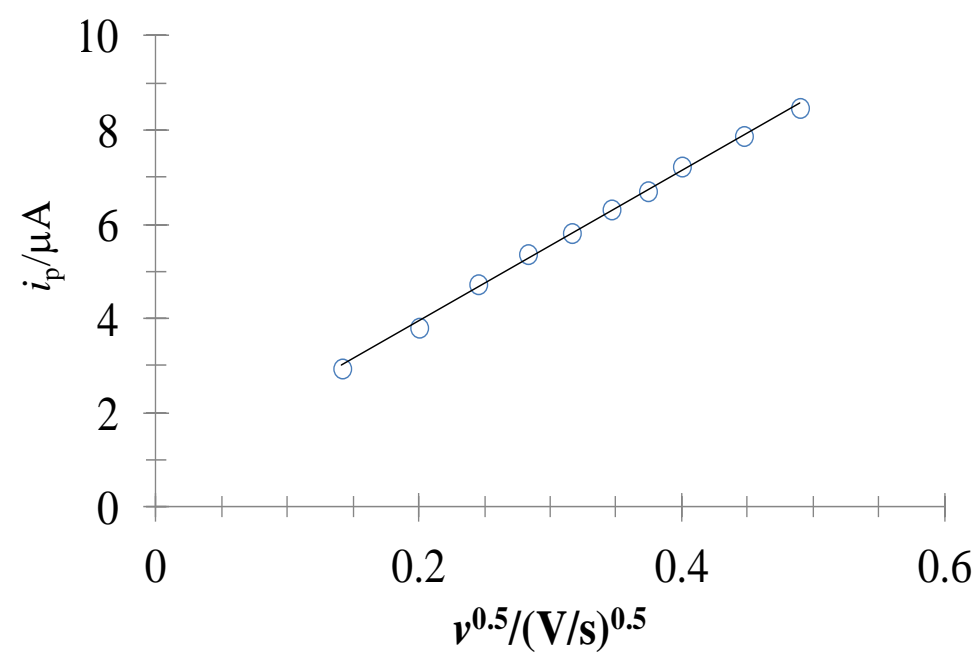

Figura 2.4. Gráfico de la $i_{p a}=f\left(\nu^{0.5}\right)$ correspondiente a la figura 2.3.

La recta de la figura 2.4 presenta la ecuación $i_{p a}(\mu \mathrm{A})=(16.04 \pm 0.26)\left(\mu \mathrm{AV}^{-0.5} \mathrm{~s}^{0.5}\right)$ $v^{0.5}+(0.731 \pm 0.088)(\mu \mathrm{A})$.

Ya que no se obtiene pico de reducción alguno y el pico de oxidación obedece a un proceso controlado por la difusión, es importante definir si se trata de un mecanismos que presenta una cinética, $k_{0}$, rápida o lenta, y/o un mecanismo químico acoplado.

Para poder describir este comportamiento es necesario conocer el número de electrones que se transfieren en la reacción electroquímica.

En la ecuación 2.1, la expresión de Randles-Sevcik, es posible apreciar que se puede determinar el número de electrones que participan en la reacción electroquímica, sin embargo se necesita del coeficiente de difusión del Tenox en medio acuoso, del cual no se conoce el valor.

Una de las técnicas electroquímicas por la cuales es posible conocer el número de electrones, prescindiendo del coeficiente de difusión, es la cronoamperometría (CA). 
2.3.2 Cálculo del número de electrones y potencial de media onda, CA.

Si bien la CA es descrita por la ecuación de Cottrel (ecuación 2.2)

$$
i=n F S C_{R} D_{R}^{1 / 2} \pi^{-0.5} t^{-0.5}
$$

Donde $F$ es la constante de Faraday, $i$ es la corriente, $n$ el número de electrones, $S$ el área del electrodo, $D_{R}$ la constante de difusión, $C_{R}$ la concentración del analíto y $t$ el tiempo.

Así entonces, utilizando la técnica experimental de CA se obtuvo una familia de potenciostatos transitorios $(i=f(t)$ ) (ver figura 2.5), para Tenox $153 \mu \mathrm{M}$ a $\mathrm{pH} 0.403$ para diferentes potenciales impuestos, los cuales van de 0.615 a $0.815 \mathrm{~V}$ que es la ventana de potencial observada para el pico de oxidación en el VC del Tenox de la figura 2.2.

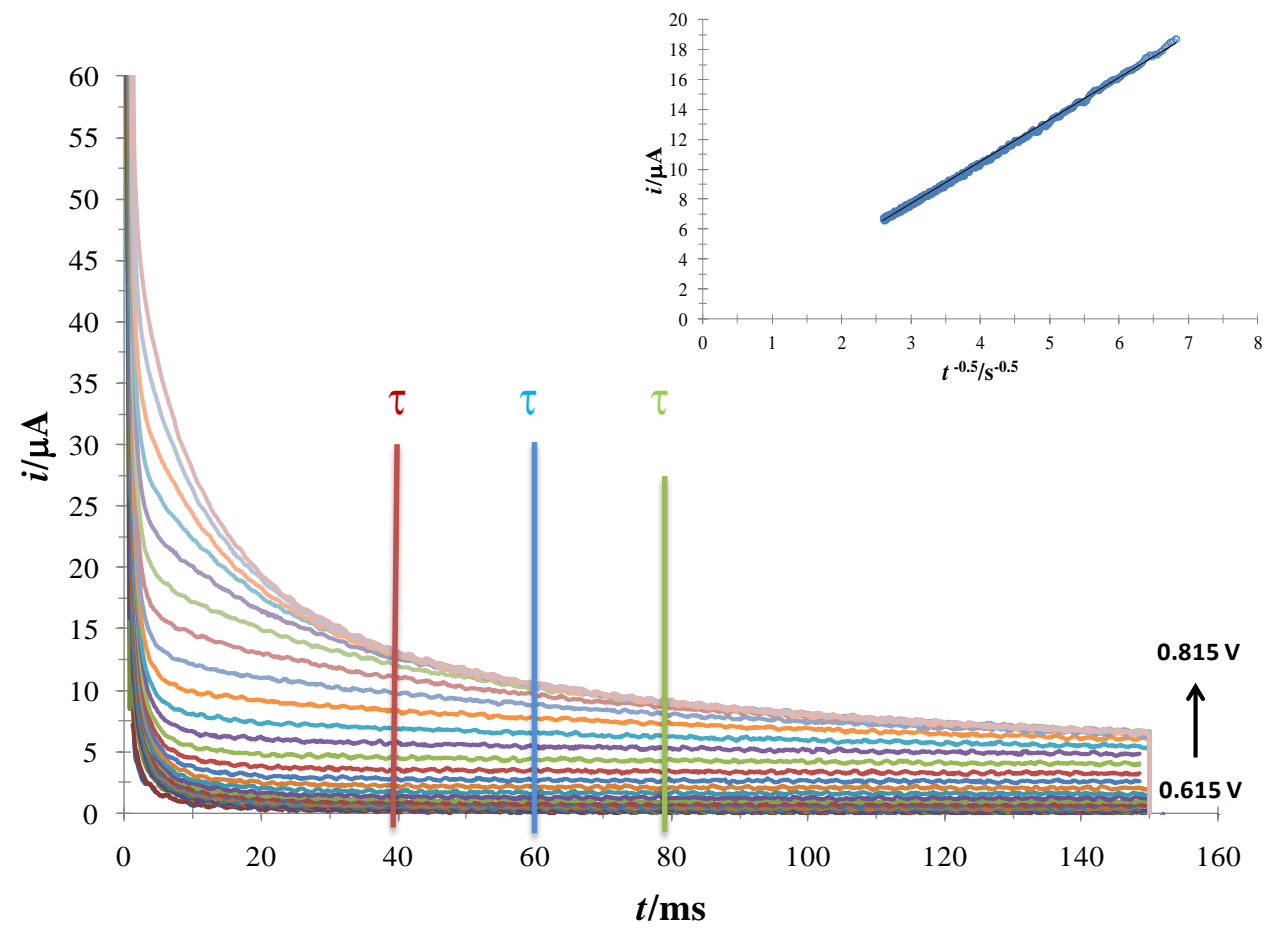

Figura 2.5. Familia de potenciostatos transitorios para diferentes potenciales mostrados en la figura en el sistema EPC/ Tenox $17 \mu \mathrm{M}$ a pH 0.410. En el inserto de la figura se presenta el gráfico de la $i=f\left(t^{-0.5}\right)$. 
Como se puede observar en el inserto de la figura 2, se tiene una relación lineal de la corriente en función del inverso de la raíz cuadrada del tiempo, la cual presenta la ecuación $\left(\mathrm{i}(\mu \mathrm{A})=(2.803 \pm 0.003)\left(\mu \mathrm{As}^{-1 / 2}\right) \mathrm{t}^{1 / 2}-(0.716 \pm 0.014)(\mu \mathrm{A})\right)$. Este comportamiento es predecible por la ecuación 2.2 de Cottrell, la cual confirma que la transferencia de carga está controlada por la difusión.

De la familia de cronoamperogramas de la figura 2.5 es posible tomar diferentes tiempos de muestreo $\tau$ y se construyen gráficas de corriente en función del potencial (ver figura 2.6). Para este sistema es válida la ecuación $2.3^{[2.4]}$.

$$
E=E_{1 / 2}-\frac{0.05916}{n} \log \left(\frac{i_{l}-i}{i}\right)
$$

donde $\mathrm{E}$ es el potencial del electrodo, $\mathrm{E}_{1 / 2}$ es el potencial de onda media, $\mathrm{i}_{1}$ es la corriente límite, $\mathrm{T}$ es la temperatura absoluta y $\mathrm{R}$ es la constante de los gases.

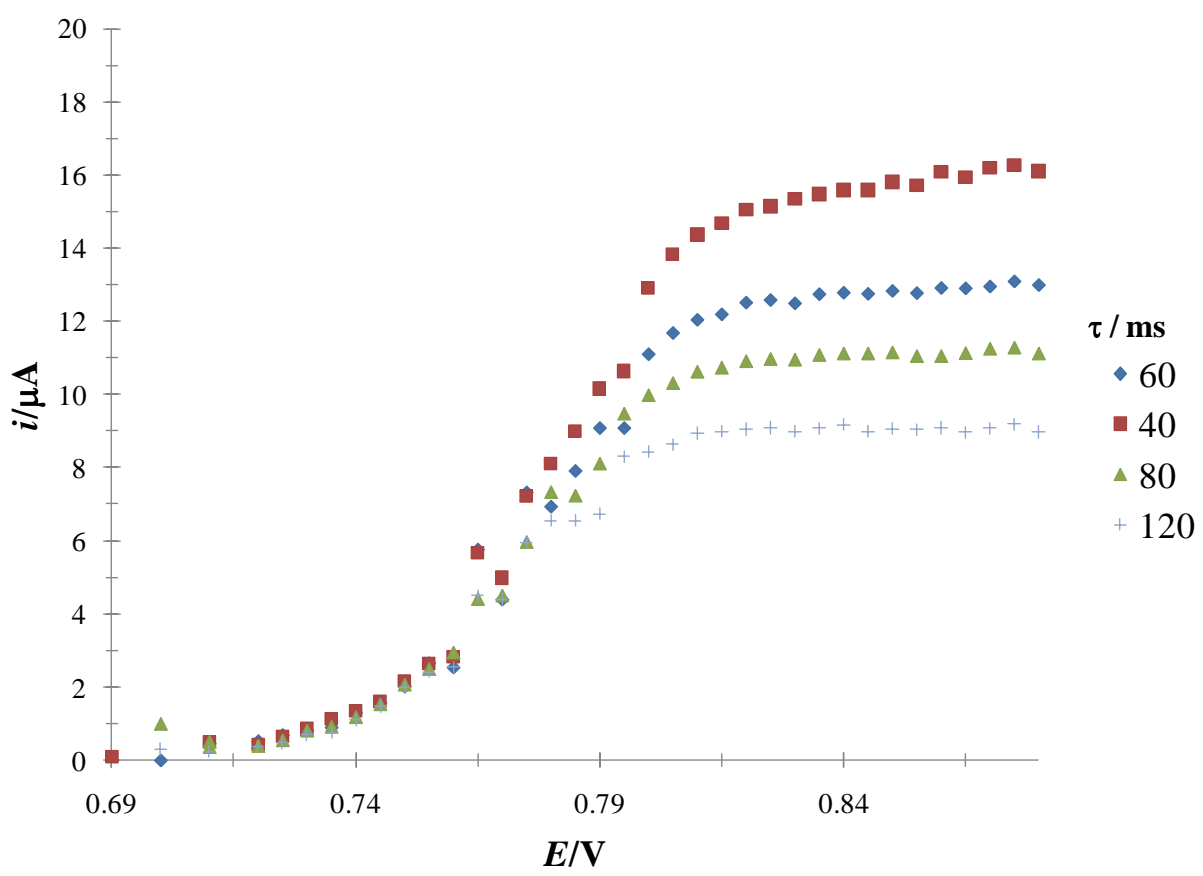

Figura 2.6. Curvas corriente-potencial para diferentes $\tau$ obtenidas a partir de los potenciostatos transitorios de la figura 2.5 
De la ecuación (2.3), es posible obtener el gráfico de $E v s \ln \left[\left(i_{l}-i\right) / i\right]$ (figura 2.7), donde se aprecia la curva de $E=f\left(\log \left(i_{L}-i / i\right)\right)$ para un $\tau$ de 60 milisegndos con ecuación $\left(\mathrm{E}(\mathrm{V})=(-0.0310 \pm 0.0004)(\mathrm{V}) \log \left[\left(\mathrm{i}_{1}-\mathrm{i}\right) / \mathrm{i}\right]+(0.7704 \pm 0.0004(\mathrm{~V}))\right.$

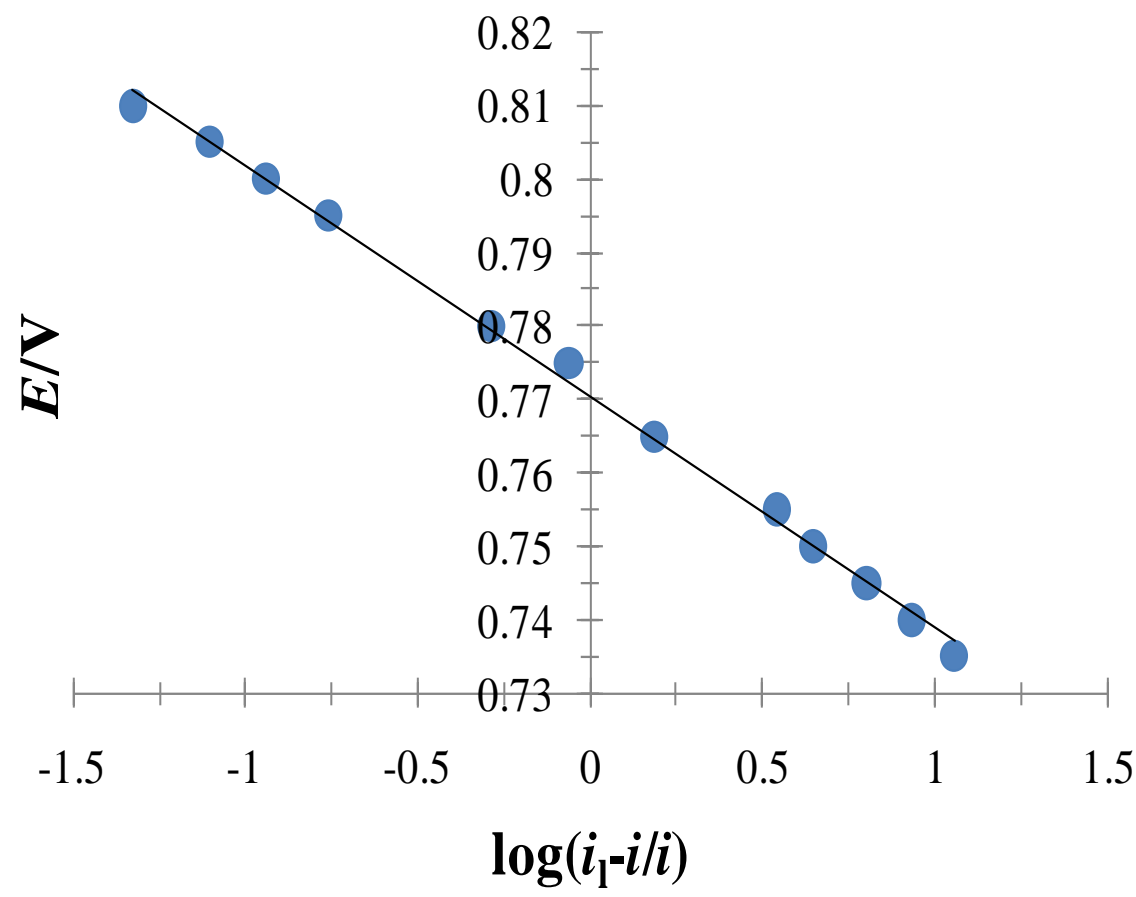

Figura 2.7. Curva $E=f\left(\log \left(i_{L^{-}}-i / i\right)\right)$ para un $\tau$ de 60 milisegundos.

De la curva de la figura 2.7 es posible obtener los valores de n y $E_{1 / 2}$. En la Tabla 2.1, es posible apreciar los valores calculados de $n$ y $E_{1 / 2}$ para distintos tiempos $\tau$.

Tabla 2.1. Cálculo de número de electrones y $\mathrm{E}_{1 / 2}$ para diferentes $\tau$ obtenidos a partir de las curvas $E=f\left(\log \left(i_{L^{-}} i / i\right)\right)$.

\begin{tabular}{|c|c|c|c|}
\hline$\tau / \mathbf{m s}$ & $\mathbf{n}$ & $\mathbf{E}_{\mathbf{1} / 2} / \mathbf{V}$ & Pendiente/V \\
\hline 40 & $2.103 \pm 0.050$ & $0.77401 \pm 0.0006$ & $-0.0281 \pm 0.0007$ \\
\hline 60 & $2.006 \pm 0.032$ & $0.77759 \pm 0.00017$ & $-0.0294 \pm 0.0005$ \\
\hline 80 & $1.905 \pm 0.026$ & $0.76564 \pm 0.00037$ & $-0.0310 \pm 0.0004$ \\
\hline 120 & $1.936 \pm 0.028$ & $0.76703 \pm 0.00034$ & $-0.0305 \pm 0.0004$ \\
\hline
\end{tabular}


Así entonces los valores obtenidos para este a cantidades fueron $\mathrm{n}=1.996 \pm 0.097 \mathrm{y}$ $\mathrm{E}_{1 / 2}=0.770 \pm 0.004$.

2.3.3 Número de electrones, (VDP).

Con el fin de confirmar el valor de $\mathrm{n}$, se utiliza la técnica de voltamperometría diferencial de pulso VDP ya que de acuerdo con Parry y Osteryoung ${ }^{[2.5]}$ y Brett y Brett ${ }^{[2.6]}$ cuando $\Delta \mathrm{E}$ se aproxima a cero se cumple (2.4), por lo tanto, el número de electrones se puede estimar usando (2.4) con $\mathrm{T}=25^{\circ} \mathrm{C}$.

$$
W_{1 / 2}=3.52 R T / n F
$$

Donde $\mathrm{W}_{1 / 2}$ es la amplitud de onda media, $\mathrm{R}$ es la constante universal de los gases, $\mathrm{n}$ es el número de electrones y F es la constante de Faraday.

En la figura 2.8 se muestra el VDP bajo condiciones estacionarias, en el sistema EPC/HCl 0.1M, Tenox $7 \mu \mathrm{M}(\mathrm{pH}=0.403)$ para diferentes amplitudes de pulso, $\Delta \mathrm{E}$.

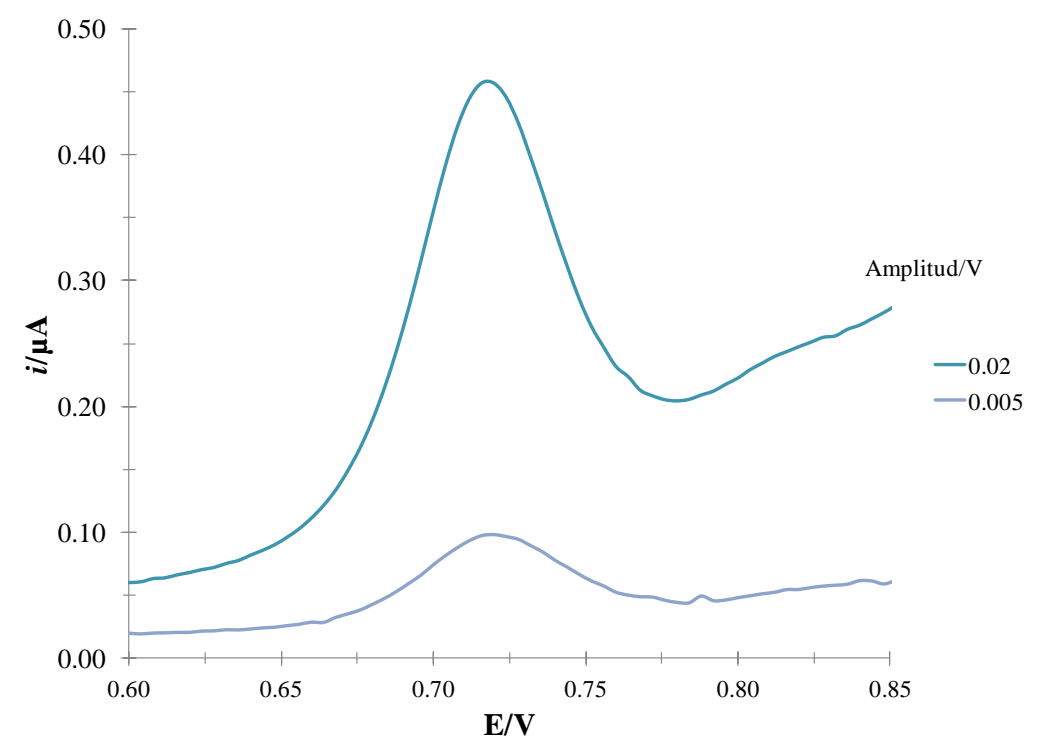

Figura 2.8. Gráfico de VDP para el sistema $\mathrm{EPC} / \mathrm{HCl}$ 0.1M, Tenox $7 \mu \mathrm{M}$ $(\mathrm{pH}=0.403)$ para dos diferentes amplitudes de pulso, $\Delta \mathrm{E}$, indicados en la figura en $\mathrm{V}$.

Para diferentes amplitudes de pulso, $\Delta \mathrm{E}$, se tienen diferentes alturas de pico medio, con las culaes es posible calcular $\mathrm{W}_{1 / 2}$. En la tabla 2 se puede observar el número de electrones, $\mathrm{n}$, obtenido para las diferente amplitudes de pulso y $\mathrm{W}_{1 / 2}$. 
Tabla 2.2. Número de electrones obtenidos y amplitud de onda media a partir de la ecuación 2.4.

\begin{tabular}{|c|c|c|}
\hline $\boldsymbol{\Delta} \boldsymbol{E} / \mathbf{V}$ & $\boldsymbol{W}_{1 / 2} / \mathbf{V}$ & $\boldsymbol{n}$ \\
\hline 0.050 & $0.060 \pm 0.001$ & $1.507 \pm 0.025$ \\
\hline 0.040 & $0.055 \pm 0.001$ & $1.64 \pm 0.029$ \\
\hline 0.030 & $0.052 \pm 0.001$ & $1.73 \pm 0.033$ \\
\hline 0.025 & $0.050 \pm 0.001$ & $1.80 \pm 0.036$ \\
\hline 0.020 & $0.048 \pm 0.001$ & $1.88 \pm 0.039$ \\
\hline 0.010 & $0.046 \pm 0.001$ & $1.96 \pm 0.042$ \\
\hline 0.005 & $0.045 \pm 0.001$ & $2.00 \pm 0.044$ \\
\hline
\end{tabular}

Como se puede observan en la Tabla 2.2, el número de electrones transferidos se aproxima a $\mathrm{n}=2$ cuando $\Delta \mathrm{E}$ tiende a cero, lo que está de acuerdo con el valor estimado en los experimentos de voltamperomentria para las curva de corriente-potencial.

Ya que se ha obtenido el número de electrones es posible adentrarse en el cálculo de $k_{0}$ para ello se utiliza voltamperometría lineal de disco rotatorio (DR).

2.3.4 Respuesta de Tenoxicam en condiciones de convección forzada, voltamperometría lineal de disco rotatorio. (DR)

Conociendo el número de electrones, es posible calcular la constante de difusión, sin embargo, como se mencionó anteriormente para ello es necesario saber qué tipo de reacción se tiene en el sistema. Por ello es importante el cálculo de la constante heterogénea de velocidad, $k_{0}$; para determinar esta constante se realizaron experimentos utilizando la técnica experimental de electrodo de disco rotatorio.

En la figura 2.7 se muestra los voltamperogramas lineales de disco rotatorio, VDR, en el sistema EPC-EDR/ $\mathrm{HCl} 0.1 \mathrm{M}$, Tenox $17 \mu \mathrm{M}(\mathrm{pH}=0.430)$ bajo condiciones de convección forzada. Los VDR fueron llevados a cabo en una ventana de potencial de 0V a $1 \mathrm{~V}$ aplicando diferentes velocidades de barrido $(v)\left(0.02 \mathrm{~V} \mathrm{~s}^{-1} \leq v \leq 1.80 \mathrm{~V} \mathrm{~s}^{-1}\right)$ y variando 
la velocidad de rotación, $\omega$, en el rango de $50 \mathrm{rpm}$ a $600 \mathrm{rpm}$. En todos los casos, el barrido de potencial se inició en potencial de circuito abierto $\left(\mathrm{E}_{\mathrm{i}}=0\right)$.

Es posible observar en todos los casos, que después de la formación de un pico bien definido, se alcanza una corriente límite anódica, $i_{l}$. Para una velocidad angular dada, $\omega$ del EPC-EDR (véase la figura 2.7(a)) el pico de corriente formado antes de la corriente límite, $i_{l}$, disminuyen conforme disminuye la velocidad de barrido de potencial, sin embargo, los valores de la corriente límite son prácticamente independientes de la velocidad de barrido del potencial. Una vez estudiado este comportamiento se obtuvieron los voltamperogramas lineales para una velocidad de barrido de $0.1 \mathrm{Vs}^{-1}$ variando la velocidad de rotación del electrodo, $\omega$, ver figura 2.7 (b).
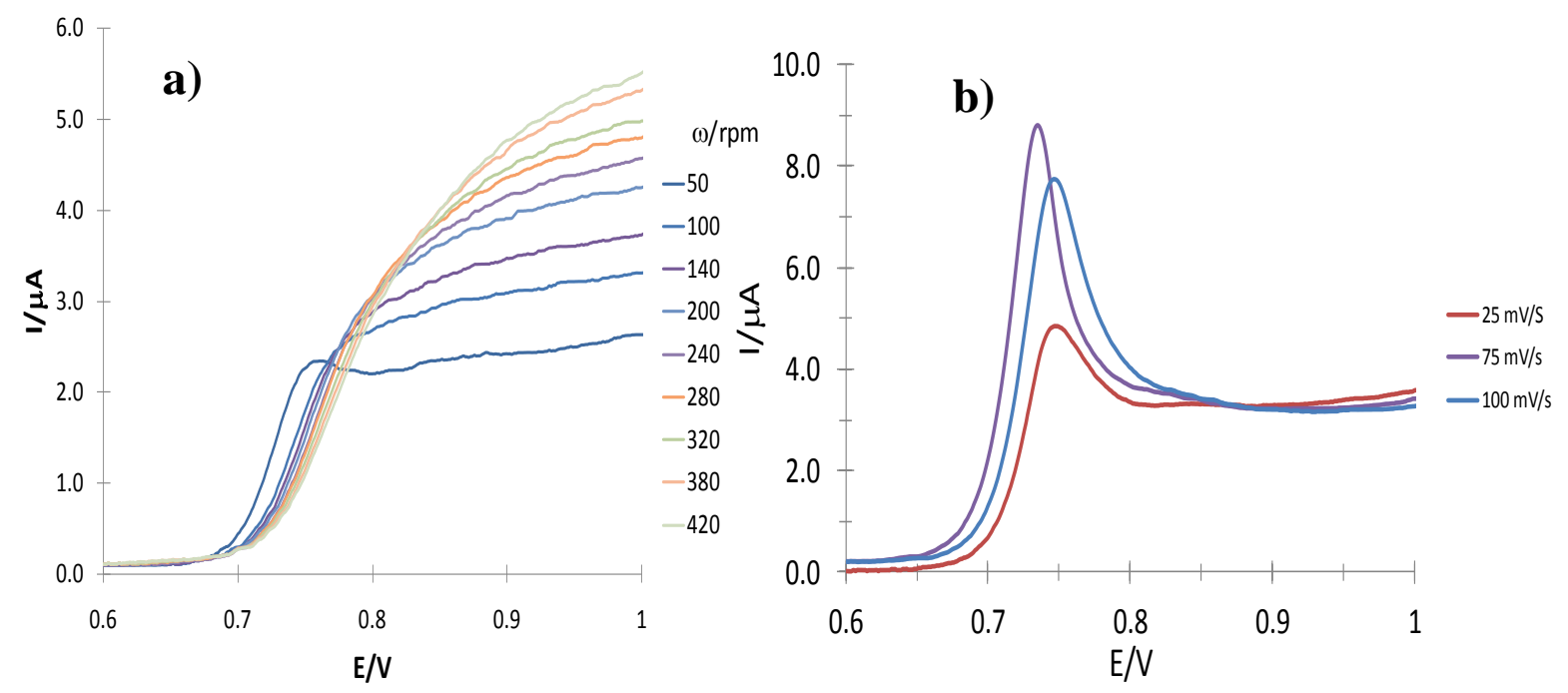

Figura 2.9. Familia de voltamperogramas de barrido lineal, bajo condiciones de convección forzada, en el sistema EPC/ $\mathrm{HCl} 0.1 \mathrm{M}$, Tenox Tenox $17 \mu \mathrm{M}(\mathrm{pH}=0.403)$ para diferentes condiciones experimentales (a) Velocida de rotación constante, w, del EPC-EDR (50 rpm) y diferentes barridos de potencial indicados en la figura; (b) diferentes valores de $\omega$, indicados en la figura en rpm. En todos los casos la velocidad de barrido de potencial es de $0.1 \mathrm{Vs}^{-1}$.

En todos los casos, después de la formación del pico de corriente anódica, se observa la corriente límite anódica. En la figura 2.10 es posible observar que la corriente límite anódica se incrementa proporcionalmente con $\omega^{0.5}$. Este comportamiento es descrito por la ecuación de Levich (2.5) que se aplica para una condición total de transferencia limitada por la masa al electrodo de disco rotatorio, EDR, y predice que la corriente límite es proporcional a $\omega^{1 / 2}$. 


$$
\mathrm{i}_{\mathrm{l}, \mathrm{a}}=0.2 \mathrm{nFSD}_{\mathrm{R}}^{2 / 3} \mathrm{C}_{\mathrm{R}} \mathrm{v}^{-1 / 6} \omega^{1 / 2}
$$

Donde $v$ es la velocidad cinemática, $\omega$ es la velocidad de rotación, $F$ es la constante de Faraday, $i_{l}$ es la corriente límite, $n$ el número de electrones, $S$ el área del electrodo, $D_{R}$ el coeficiente de difusión y $C_{R}$ la concentración del analito.

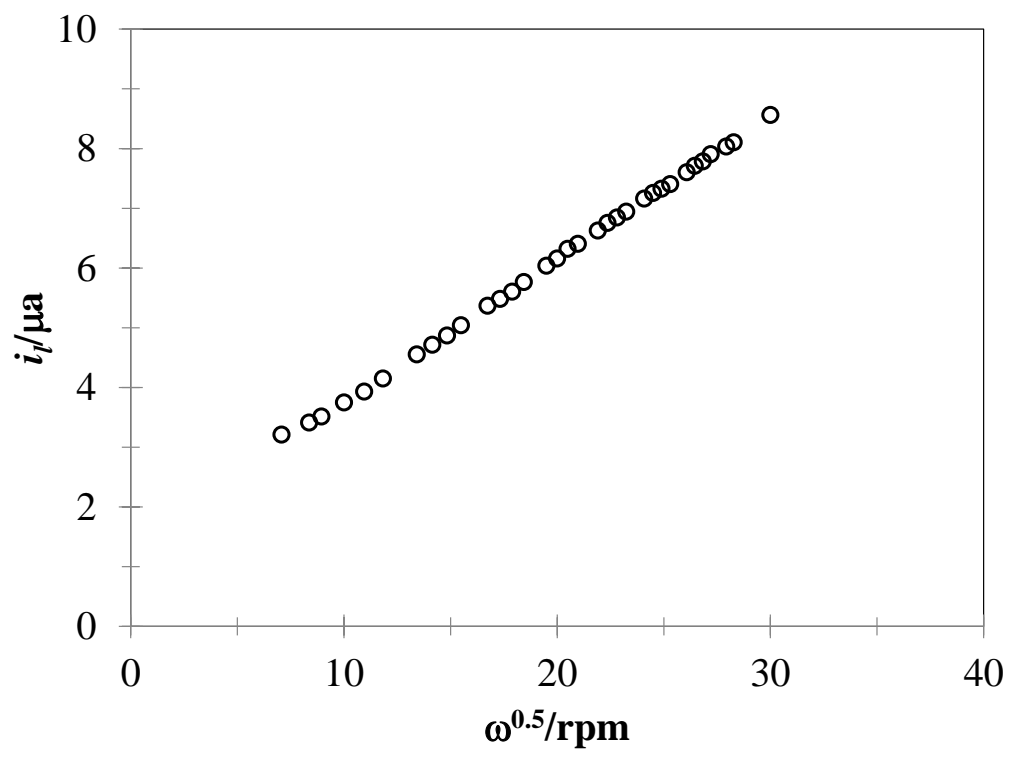

Figura 2.10. Corriente límite evaluada a $0.9 \mathrm{~V}$ (puntos) en función de $\omega^{0.5}$; el ajuste de la recta $\left(\mathrm{i}_{1}(\mu \mathrm{A})=(2.43 \pm 0.02) 10^{-7}\left(\mu \mathrm{Arpm}^{-0.5}\right) \omega^{0.5}+(1.55 \pm 0.05) 10^{-6} 10^{-7}(\mu \mathrm{A})\right)$.

Utilizando la ecuación de Koutecky-Levich (2.6) ${ }^{[2.4]}$, se obtuvieron para diferentes potenciales las rectas del inverso de la corriente en función del inverso de la velocidad de rotación a la un medio

$$
\frac{1}{i}=\frac{1}{i_{k}}+\frac{1}{0.62 n S F D_{R}^{2 / 3} C_{R} v^{-1 / 6} \omega^{0.5}}
$$

En la ecuación 2.6 es posible observar que la pendiente es el inverso de la ecuación de Levich y la ordenada es el inverso de la corriente cinética. 
En la figura 2.11 se muestran estas rectas dadas por la ecuación 2.6 las cuales ilustran dos potenciales de $0.85 \mathrm{~V}$ y $1 \mathrm{~V}$.

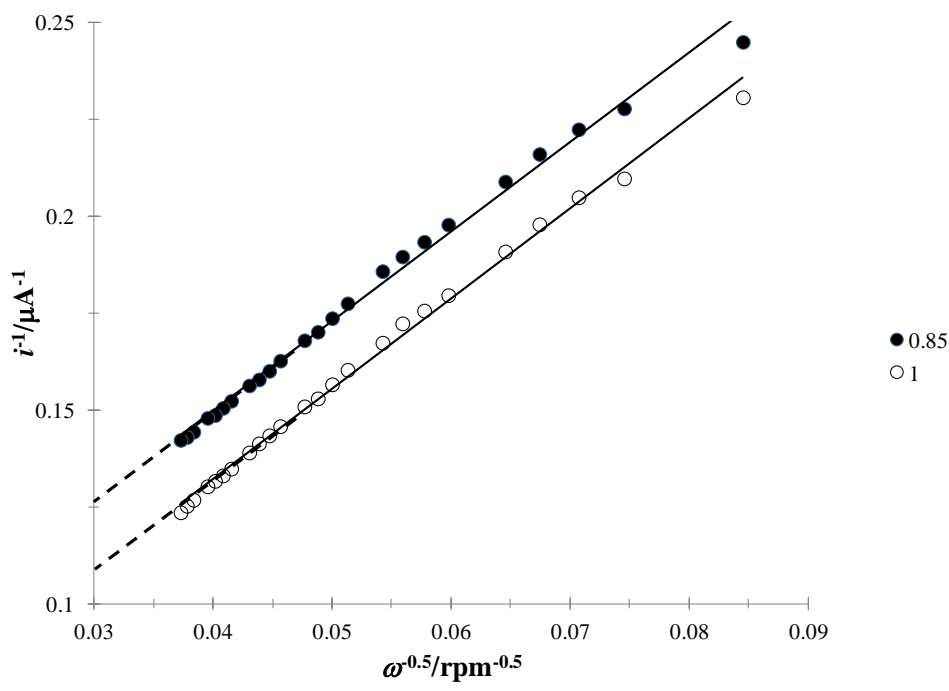

Figura 2.11. Curvas de Koutecky-Levich para potencial de 1 y $0.85 \mathrm{~V}$ para el sistema EPC/HCl 0.1M, Tenox Tenox $(153 \pm 1) \mathrm{mM}(\mathrm{pH}=0.403)$.

A partir de la corriente cinética y siguiendo la ecuación 2.7 de Tafel es posible obtener el valor de $\mathrm{K}_{0}$.

$$
\begin{aligned}
& i_{0}=F A k_{0} C \\
& k_{0}=\frac{i_{0}}{F A C}
\end{aligned}
$$

Así se obtiene un valor para la constante de velocidad heterogénea $k_{0}$ de $0.09 \mathrm{cms}^{-1}$. Esto es indicativo de que se tiene en el sistema una reacción irreversible con una constante heterogénea de velocidad $k_{0}$ grande y una reacción química acoplada con una velocidad de reacción grande. 


\subsubsection{El coeficiente de difusión del Tenoxicam.}

Una vez caracterizado el pico de oxidación de Tenox y obtenidos los valores fisicoquímicos asociados a éste, es posible calcular el coeficiente de difusión de la molécula en nuestro sistema. Esta determinación se lleva a cabo mediante las técnicas experimentales electroquímicas y mediante cálculos computacionales.

\subsubsection{Determinación experimental del coeficiente de difusión.}

Una vez conocido el número de electrones transferidos durante la oxidación del Tenox, es posible calcular el coeficiente de difusión a partir de la pendiente de la recta de acuerdo a la expresión de Randles-Sevcik (2.1) para una cinética rápida, la de cottrell (2.2), y la de Levich (2.5), los resultados se pueden apreciar en la tabla 2.3

Tabla 2.3. Determinación de constante de difusión obtenida por diferentes técnicas electroquímicas y teórico considerando un valor de $\mathrm{n}=1.996 \pm 0.097$.

\begin{tabular}{|c|c|c|}
\hline Técnica & Pendiente & $\mathbf{1 0}^{\mathbf{6}} \mathbf{D} / \mathbf{c m}^{2} \mathbf{s}^{-1}$ \\
\hline VC & $(16.04 \pm 0.26)\left(\mathrm{mAV}^{-0.5} \mathrm{~s}^{0.5}\right)$ & $3.745 \pm 0.077$ \\
\hline CA & $(2.405 \pm 0.028)\left(\mathrm{mAs}^{-0.5}\right)$ & $4.116 \pm 0.086$ \\
\hline DR & $(2.43 \pm 0.02)\left(\mathrm{mArpm}^{-0.5}\right)$ & $4.396 \pm 0.058$ \\
\hline Cálculo Teórico & - & 4.54 \\
\hline
\end{tabular}

Así el valor promedio de D experimental es de $(3.87 \pm 0.47) \times 10^{-6} \mathrm{~cm}^{2} \mathrm{~s}^{-1}$.

\subsubsection{Determinación computacional del coeficiente de difusión.}

El valor teórico del coeficiente de difusión del Tenox es calculado a partir de la aproximación de Einstein-Stokes ${ }^{[2.7-2.9]}$ :

$$
D=\frac{k_{B} T}{6 \pi \eta a}
$$

Donde $\boldsymbol{k}_{\boldsymbol{B}}$ es la constante de Boltzman, T es la temperatura a $298 \mathrm{~K}$, $\boldsymbol{\eta}$ denota la viscosidad del solvente, que en este caso es agua $\left(\eta=8.91 \times 10^{-4}\right)$ y a es el radio del soluto. El valor de a fue estimado usando el paquete de programas computacionales Gaussian $03^{[2.10]}$, con la opción de volumen=ajustado. La geometría para esta estimación fue previamente optimizada con un nivel de teoría M052X/6-31+g(d,p). Es importante notar 
que el valor de $\mathrm{D}$ es similar a los valores experimentales evaluados bajo condiciones estacionarias y de convección forzada hidrodinámicas.

Cabe mencionar que la mejor técnica para obtener el coeficiente de difusión con el menor error estadístico es la de voltamperometría lineal de disco rotatorio (DR), sin embargo en la tabla 2.3 es posible observar que el valor del coeficiente de difusión obtenido a partir de las otras técnicas es muy cercano entre sí.

Así mismo es importante hacer notar que el valor del coeficiente de difusión obtenido es del orden de la sacarosa en agua.

2.3.5 Estudio electroquímico para las especies de HTenox (z) y Tenox-.

El estudio de caracterización electroquímica que se llevo a cabo para la especie protonada de $\mathrm{H}_{2}$ Tenox ${ }^{+}$, se realizó también para las especies de HTenox (z) (pH 3.175), y Tenox ${ }^{-},(\mathrm{pH} 7.150)$.

En la Figura 2.12 se muestran VCs obtenidos en el sistema EPC / $17 \mu \mathrm{M}$ Tenox, $0.2 \mathrm{M} \mathrm{Cl}^{-}$para las diferentes especies de Tenox: $\mathrm{H}_{2}$ Tenox $\left.{ }^{+}(\mathrm{pH} 0.405), \mathrm{HTenox} \mathrm{z}\right)(\mathrm{pH}$ 3.175), y Tenox ${ }^{-},(\mathrm{pH} 7.150)$.

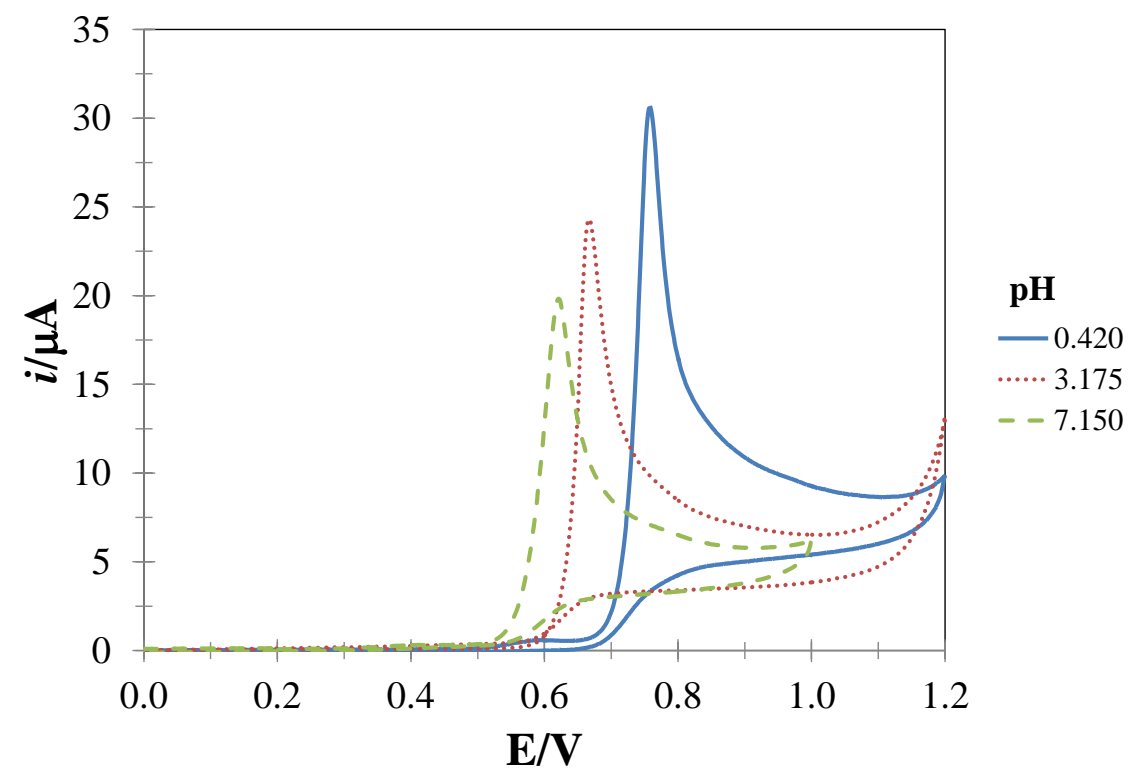

Figura 2.12. Familia de VCs obtenidos en el sistema EPC / $17 \mu \mathrm{M}$ Tenox, $1 \mathrm{M} \mathrm{Cl}^{-}$ con diferentes valores de $\mathrm{pH}$ mostrados en la Figura. En todos los casos el barrido de potencial comenzó a $0 \mathrm{~V}$ en dirección anódica a una velocidad de barrido de potencial de $0.1 \mathrm{Vs}^{-1}$ 
Para los tres casos el barrido de potencial se inicia en potencial de circuito abierto $\left(\mathrm{E}_{\mathrm{i}}=0\right)$ hacia potenciales positivos a una velocidad de barrido de potencial de $0.1 \mathrm{Vs}^{-1}$ y es posible apreciar que se presenta un pico de oxidación en $0.78 \mathrm{~V}, 0.667 \mathrm{~V}$ y $0.610 \mathrm{~V}$. Al invertir el barrido de potencial se espera observar el pico de reducción correspondiente al pico anódico presente, sin embargo para las tres especies no se presenta pico de reducción alguno. Así mismo se aprecia que el potencial de oxidación disminuye al aumentar el pH; éste mismo efecto se puede observar con la corriente asociada.

Al presentar, la especie aniónica y neutra, el mismo comportamiento electroquímico que la especie protonada, se lleva a cabo el estudio para determinar el número de electrones y $k_{0}$ con lo que se tiene que para estas especies el número de electrones transferidos también es de dos y presentan una cinética electroquímica rápida con una reacción química acoplada.

Así mismo se determinó el coeficiente de difusión para la especie HTenox (z), y Tenox ${ }^{-}$; este valor se compara en la tabla 2.4.

Tabla 2.4. Determinación de la constante de difusión obtenida para las diferentes especies de Tenox utilizando la técnica de DR, considerando un valor de $n=1.996 \pm 0.097$.

\begin{tabular}{|l|l|}
\hline Técnica DR & $\mathbf{1 0}^{6} \mathbf{D} / \mathbf{c m}^{2} \mathbf{s}^{-1}$ \\
\hline $\mathrm{H}_{2}$ Tenox $^{+}$ & $4.396 \pm 0.058$ \\
\hline HTenox(z) & $4.405 \pm 0.058$ \\
\hline Tenox $^{-}$ & $4.630 \pm 0.058$ \\
\hline
\end{tabular}

Es posible apreciar en la tabla 2.4 que el coeficiente de difusión obtenido para las distintitas especies de Tenox no es el mismo. Sin embargo es cercano ya que la diferencia de especie a especie es la pérdida de un protón. 


\subsection{Conclusiones.}

Se determinó en la caracterización electroquímica de las tres especies de Tenox que no presentan picos de reducción asociados al pico de oxidación; además se estimó que durante su oxidación electroquímica participan 2 electrones y se encontró que la oxidación electroquímica para las tres especies Tenox es un proceso controlado por la difusión que sigue un mecanismo electroquímico-químico (EC por sus siglas en inglés) donde la cinética electroquímica y química son rápidas. Así mismo se determinó el coeficiente de difusión para las distintas especies del Tenox. 


\section{CAPÍTULO 3. PRODUCTOS DE OXIDACIÓN DE TENOX.}

\subsection{Introducción.}

Teniendo en cuenta que la oxidación electroquímica del Tenox es un proceso controlado por la difusión que sigue un mecanismo EC donde la cinética electroquímica y química son rápidas como se ha concluido en el capítulo 2, es conveniente determinar la naturaleza más probable de los productos no electroactivos de esta reacción. Por ello en este capítulo se presenta un estudio espectro-electroquímico y de cromatografía líquida de alta resolución (HPLC) para la caracterización de estos productos de oxidación, apoyándose con cálculos teóricos de la teoría de funcionales de la densidad (DFT)

Para el estudio de caracterización de los productos de oxidación del Tenox, se lleva a cabo una macroelectrólisis en la cual después de cada tiempo determinado de oxidación se toma la respuesta del voltamperograma cíclico, el espectro de absorción de UV-Vis y el cromatograma de HPCL de la molécula del Tenox con el fin seguir la oxidación de esta molécula. A continuación se explican los detalles del estudio experimental.

\subsection{Metodología}

\subsubsection{Reactivos.}

Todos los reactivos son de grado analítico, Tenox (FLUKA), hidróxido de sodio (JT Baker) y ácido clorhídrico (Monterrey), todas las soluciones fueron preparadas con agua desionizada.

\subsubsection{Equipo utilizado para los experimentos electroquímicos.}

Se utilizó una celda convencional de tres electrodos, empleando como electrodo de referencia un electrodo de platino platinado (BAS contraelectrod-MW-1033); como electrodo de referencia un electrodo de $\mathrm{Ag} / \mathrm{AgCl}$ (BAS-MF-2079) y como electrodo de trabajo un electrodo de pasta de carbón CPE (BAS-electrodo de trabajo MF-2065 diámetro de $3 \mathrm{~mm})$.

Los experimentos de macroelectrólisis se llevaron a cabo en una celda masiva de electrolisis (Princeton Applied Research Kit 377/16 Stirring Synchronous Motor), utilizando como electrodo de trabajo una malla de platino (MF-2077 BAS electrodo con un área superficial de $10.5 \mathrm{~cm}^{2} / \mathrm{cm}^{3}$ ), un electrodo de vidrio con puente de alambre de platino 
como electrodo de referencia (Princeton Applied Research Kit K0030) y un electrodo de pasta de carbón como contra electrodo.

3.2.4 Equipo utilizado para el experimento espectrofotométrico UV-Vis.

Los estudios espectrofotométricos se llevaron a cabo utilizando un espectrofotómetro UV-Vis Perkin Elmer Lambda 20 y una celda de cuarzo de $1 \mathrm{~cm}$ de longitud de paso óptico.

3.2.5 Equipo utilizado para el experimento de cromatografía líquida de alta resolución.

Los experimentos de cromatografía líquida de alta presión, HPLC por sus siglas en inglés, fueron realizados en un cromatógrafo Perkin Elmer series 200 de alta resolución, el cual lleva integrado un detector de arreglo de diodos con una lámpara UV-Vis la cual se fijó a $354 \mathrm{~nm}$. El inyector consiste en un bucle de $20 \mu \mathrm{L}$. La columna de trabajo es una Phenomenex Luna 5u es C18 (2) 100A. La fase móvil consistió en una mezcla de $200 \mathrm{ml}$ de metanol, $100 \mathrm{ml}$ de acetonitrilo, $10 \mathrm{ml}$ de ácido acético, $10 \mathrm{ml}$ ácido fosfórico y $200 \mathrm{ml}$ de agua. Se trabajó con un flujo de agua de $1 \mathrm{ml} / \mathrm{min}$.

\subsubsection{Oxidación de la molécula de Tenoxicam.}

La figura 3.1 muestra una familia de VCs para diferentes tiempos de oxidación, de 1 minuto a 24 minutos, tomados después de una macro electrolisis, para todos los casos se inició un barrido de potencial en corriente nula hacia potenciales positivos presentando un pico de oxidación alrededor de $0.78 \mathrm{~V}$, se observa que conforme incrementa el tiempo de oxidación el pico anódico se mueve hacia potenciales más positivos al mismo tiempo que disminuye la corriente de pico siendo así para el tiempo de 24 minutos la corriente es prácticamente nula. 


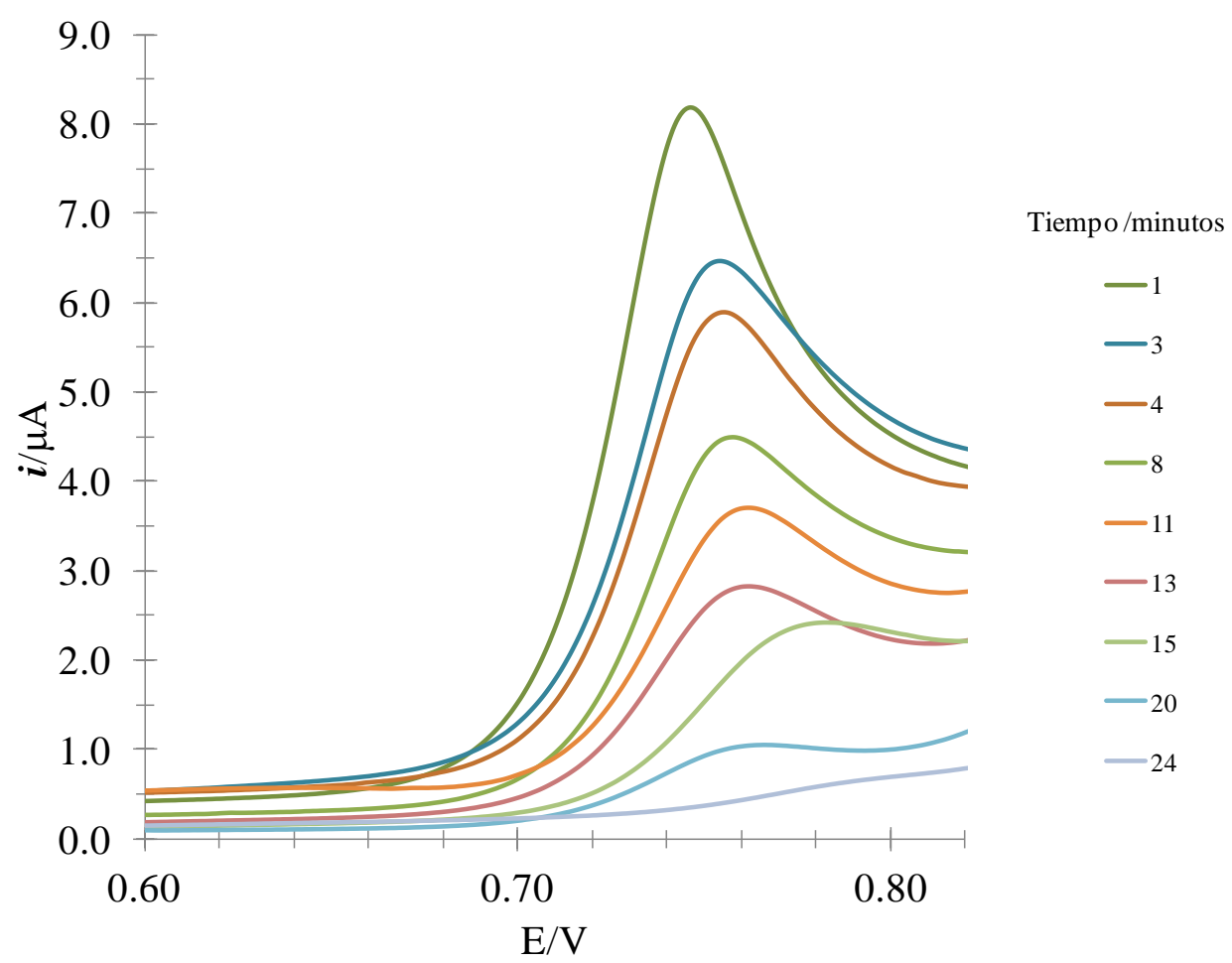

Figura 3.1. Familia de voltamperograma cíclicos aplicado para una velocidad de barrido de $0.1 \mathrm{~V} \mathrm{~s}^{-1}$, en el sistema $0.1 \mathrm{M} \mathrm{HCl},(153 \pm 1) \mu \mathrm{M}$ tenox $(\mathrm{pH}=0.461)$ después de diferentes tiempos de oxidación, para $1 \mathrm{~V}$, indicados en la figura en minutos.

En la figura 3.2 se presentan los espectros de absorción obtenidos después de imponer un potencial de oxidación durante diferentes tiempos en el sistema EPC / Tenox $(153 \pm 1) \mu \mathrm{M}, \mathrm{HCl} 0.1 \mathrm{M}(\mathrm{pH}=0.405)$. Se puede observar que el pico de absorción característico del Tenox que se presenta en $\lambda=354 \mathrm{~nm}$ disminuye a medida que el tiempo de oxidación aumenta. Así mismo se puede apreciar la formación de 3 bandas a longitudes de onda menores a 300nm mientras que en el inserto se observa el espectro de absorción obtenido para el tiempo final de oxidación, en el cual la banda característica del Tenox en $354 \mathrm{~nm}$ prácticamente no presenta absorción. 


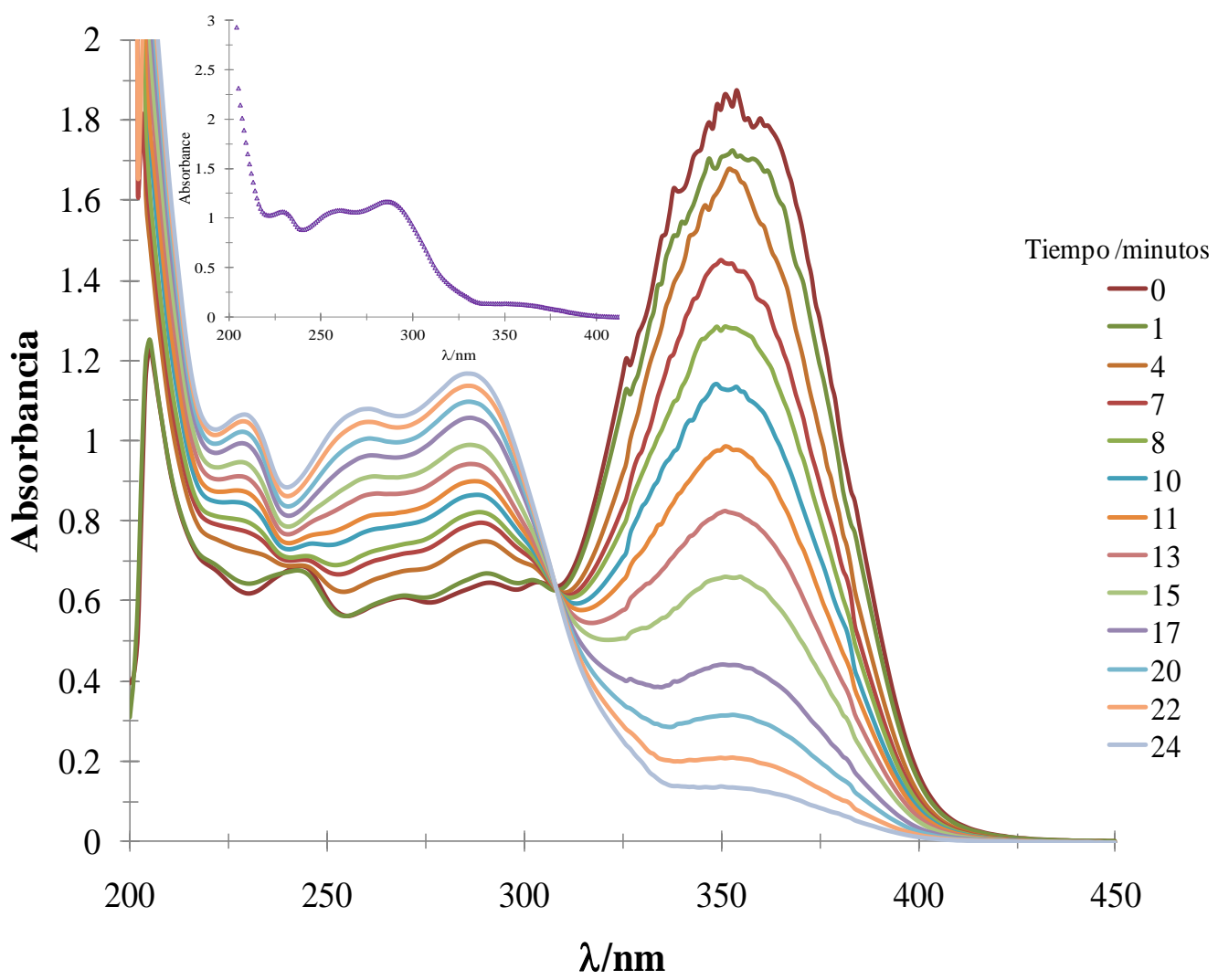

Figura 3.2. Familia de espectros UV-Vis en el sistema $0.1 \mathrm{M} \mathrm{HCl},(153 \pm 1) \mu \mathrm{M}$ Tenox $(\mathrm{pH}=0.461)$ después de diferentes tiempos de oxidación, para $1 \mathrm{~V}$, indicados en la figura en minutos.

\subsection{Estudio del mecanismo de oxidación del Tenoxicam.}

Existen diversas propuestas experimentales respecto al mecanismo de oxidación de la molécula del Tenox, por lo que se evaluaron estas propuestas con cálculos computacionales.

3.3.1 Detalles Computacionales para el estudio del mecanismo de oxidación.

Los cálculos de optimización de geometría y frecuencia se llevaron a cabo utilizando el funcional M05-2X ${ }^{[3.1]}$ y el conjunto base 6-3 1+G(d,p). Los cálculos de optimización de geometría y frecuencia fueron realizados en solución, utilizando el modelo continuo SMD ${ }^{[3.2]}$ y agua como solvente. Todos los cálculos se realizaron con el paquete de programas Gaussian $09^{[3.3]}$. Las correcciones termodinámicas a $298 \mathrm{~K}$ fueron incluidas en el cálculo de energías relativas. Los espectros calculados se han calculado utilizando la 
teoría funcional de la densidad dependiente el tiempo (TD-TFD), con transición vertical que implica hasta tres estados excitados.

3.3.2 Mecanismo de la oxidación de la molécula del Tenoxicam sin fragmentación.

De acuerdo con Tsopelas y colaboradors ${ }^{[3.4]}$ la oxidación electroquímica de los oxicams, incluido el Tenox, podría involucrar la pérdida de uno o dos electrones sin considerar los productos de oxidación de una posible fragmentación (ver esquema 3.I). Sin embargo, el mecanismo propuesto por Tsopelas no explica la irreversibilidad observada en los voltamperogramas cíclicos del Tenox.

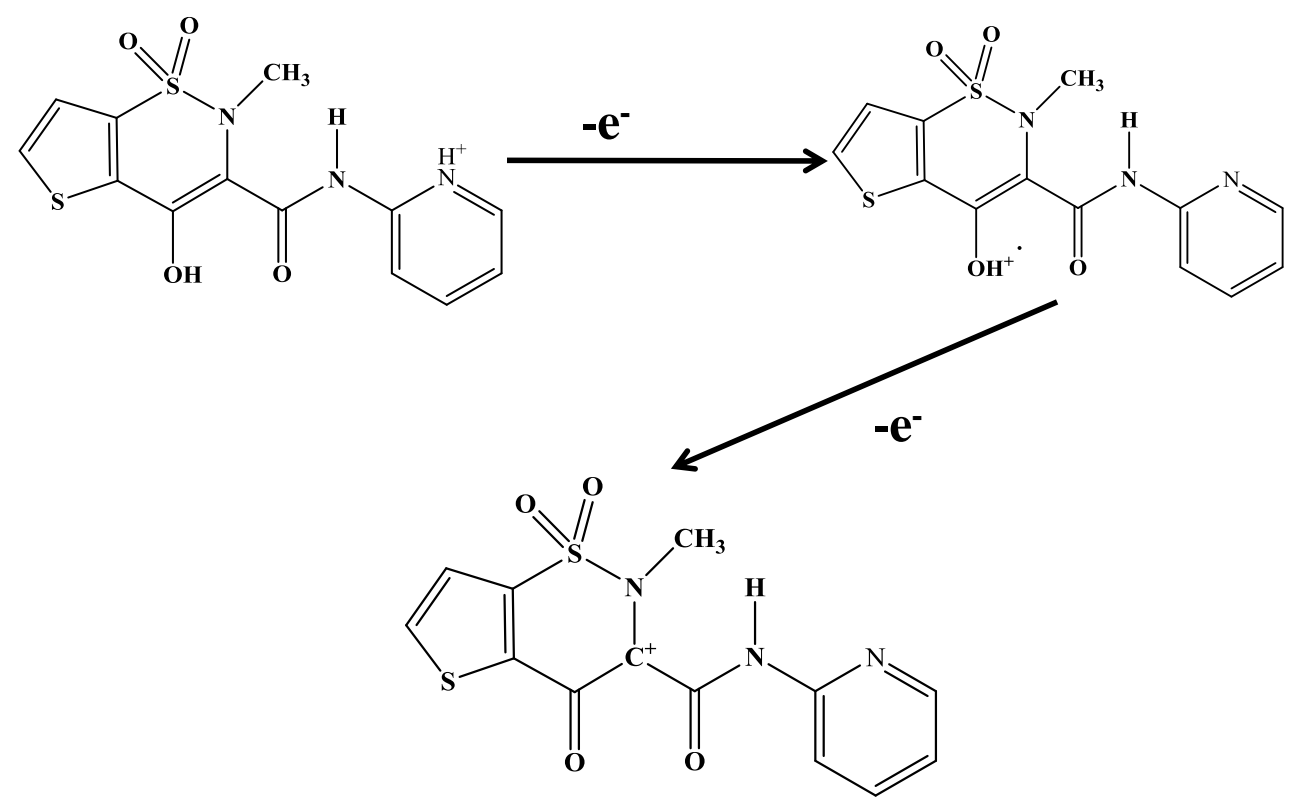

Esquema 3.I. Mecanismo de oxidación electroquímica de los oxicams de acuerdo con Tsopelas y colaboradores. ${ }^{[3.4]}$

No obstante, se calcularon los espectros UV-Vis para las dos posibles oxidaciones de la especie de $\mathrm{H}_{2} \mathrm{Tenox}^{+}$, resultando de la pérdida de un electrón el $\mathrm{H}_{2} \mathrm{Tenox}^{2+{ }^{*}}$ y de la pérdida de un segundo electrón la especie $\operatorname{HTenox}^{2+}$, sin fragmentación alguna de la molécula. En la figura 3.3 es posible notar que ambas especies, $\mathrm{H}_{2} \mathrm{Tenox}^{2+}{ }^{-}$y $\mathrm{HTenox}^{2+}$, se presentan alrededor de los $450 \mathrm{~nm}$; sin embargo en la figura 3.3 puede observarse, que incluso después de 24 minutos de macroelectrólisis no hay picos alrededor de $450 \mathrm{~nm}$. Así el mecanismo descrito en el esquema no es válido para explicar la oxidación electroquímica Tenox. 


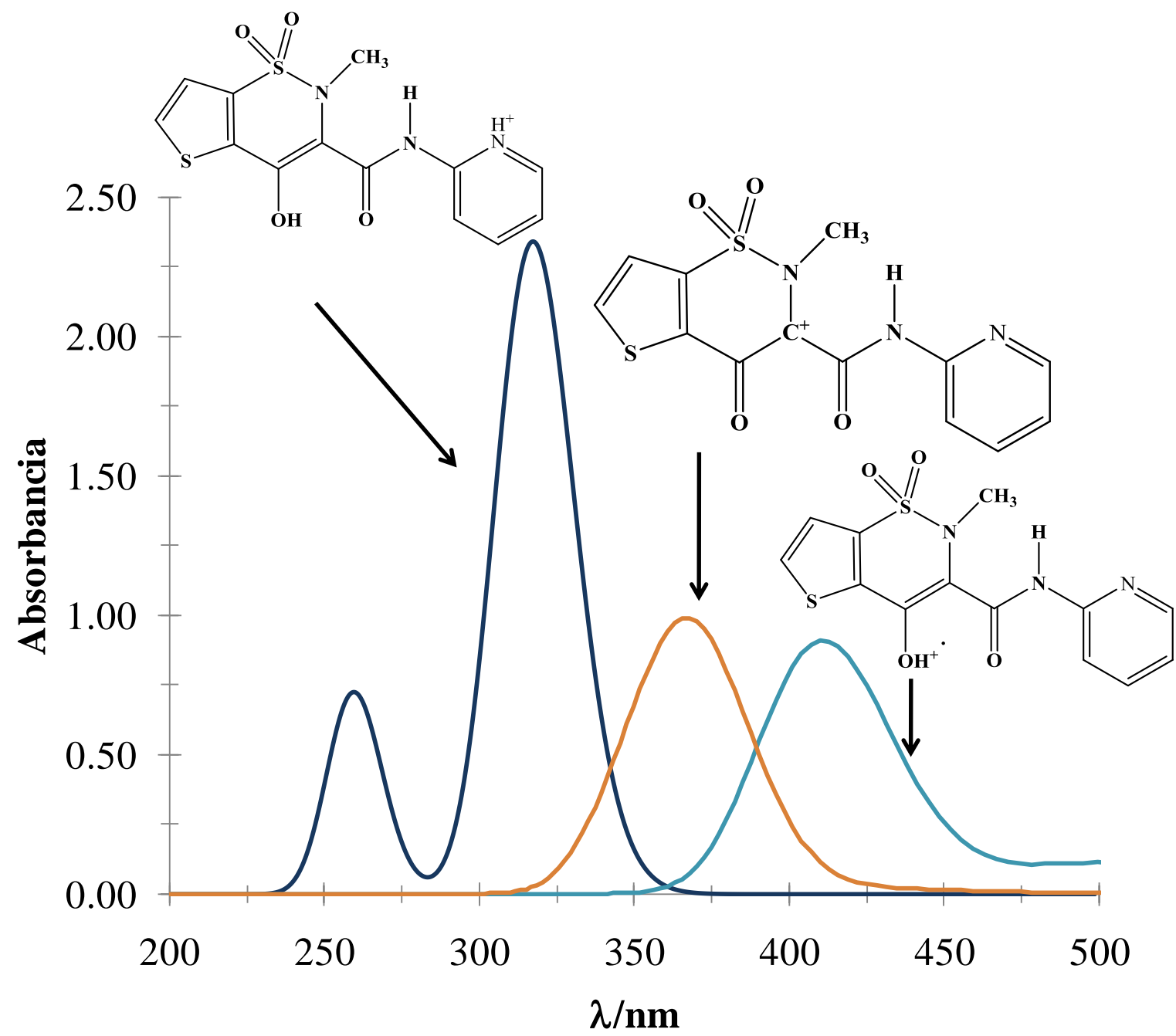

Figura 3.3. Cálculo de espectros UV-Vis para las especies de $\mathrm{H}_{2} \mathrm{Tenox}^{+}, \mathrm{H}_{2} \mathrm{Tenox}^{2+}$

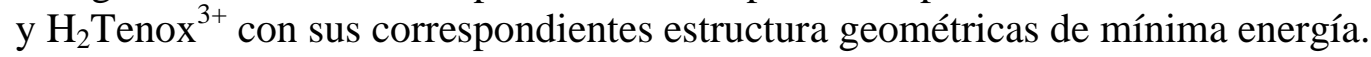

3.3.3 Mecanismo propuesto de la oxidación de la molécula del Tenoxicam con fragmentación.

En el esquema 3.II implica varias vías de fragmentación basadas en el mecanismo de oxidación propuesto por Ichihara y colaboradores. ${ }^{[3.5,3.7]}$

El esquema 3.III también involucra distintos caminos de fragmentación, pero estos corresponden a los propuestos en este estudio. En ambos esquemas se presentan las energías libres de Gibbs de la reacción a $298 \mathrm{~K}$, asociados con cada ruta propuesta en los esquemas. Los valores de $\Delta \mathrm{G}^{\circ}$ se calcularon utilizando $\Delta \mathrm{G}_{\mathrm{gas}}\left(\mathrm{H}^{+}\right)=-4.39 \mathrm{kcal} / \mathrm{mol}$ y 
$\Delta \mathrm{G}_{\text {solvatación }}\left(\mathrm{H}^{+}\right)=-256.89 \mathrm{kcal} / \mathrm{mol}$, basado sobre la recomendación de Camaioni $\mathrm{y}$ Schwerdtfeger. ${ }^{[3.8]}$

Estos valores muestran que todos los caminos tienen una reacción significativamente exergónica, por lo que se espera que todos se produzcan espontáneamente.

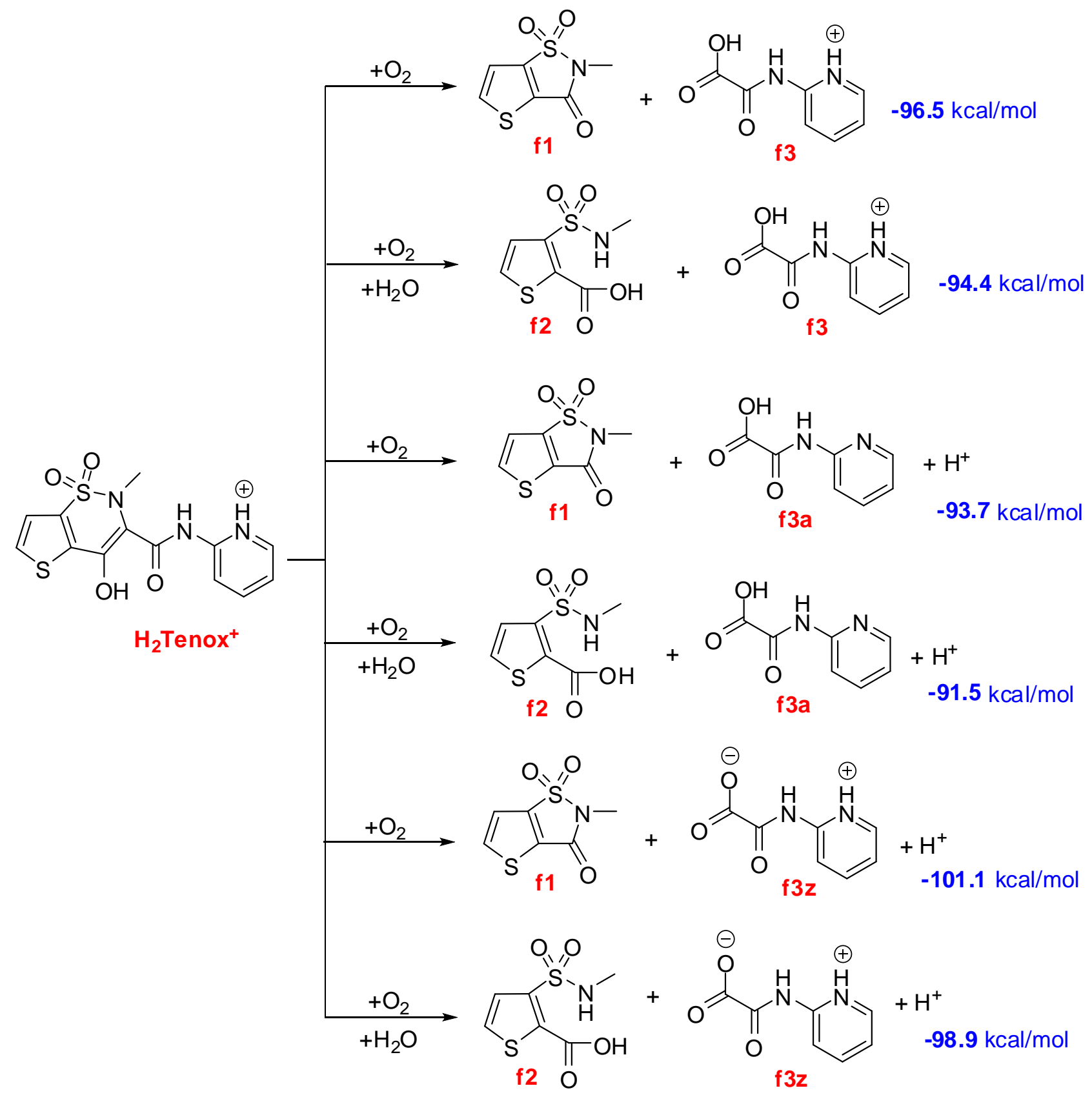

Esquema 3.II. Esquema de fragmentación, incluyendo caminos basados en el mecanismo propuesto por Ichihara y colaboradores ${ }^{[3.5,3.7]}$ 


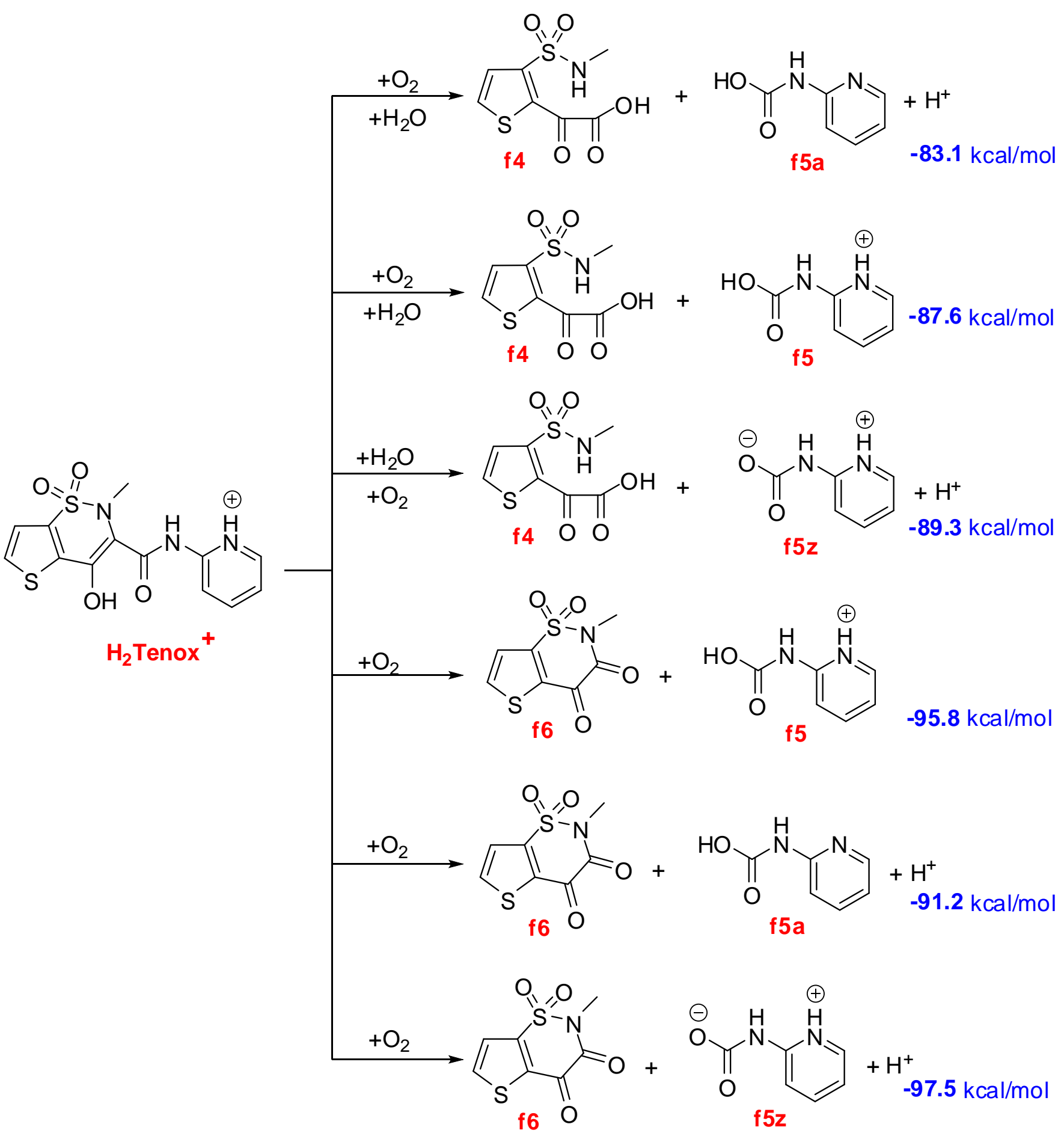

Esquema 3.III. Esquema de fragmentación, incluidos los caminos propuestos en el presente trabajo. 
En la figura 3.4 a y 3.4 b se muestran los espectros calculados correspondientes al esquema 3.II y al esquema 3.III respectivamente. Además del espectro de cada fragmento, también muestran un espectro etiquetado como total para cada esquema de fragmentación, que se obtuvo usando la aditividad de absorbancia y la abundancia relativa de los fragmentos formados.
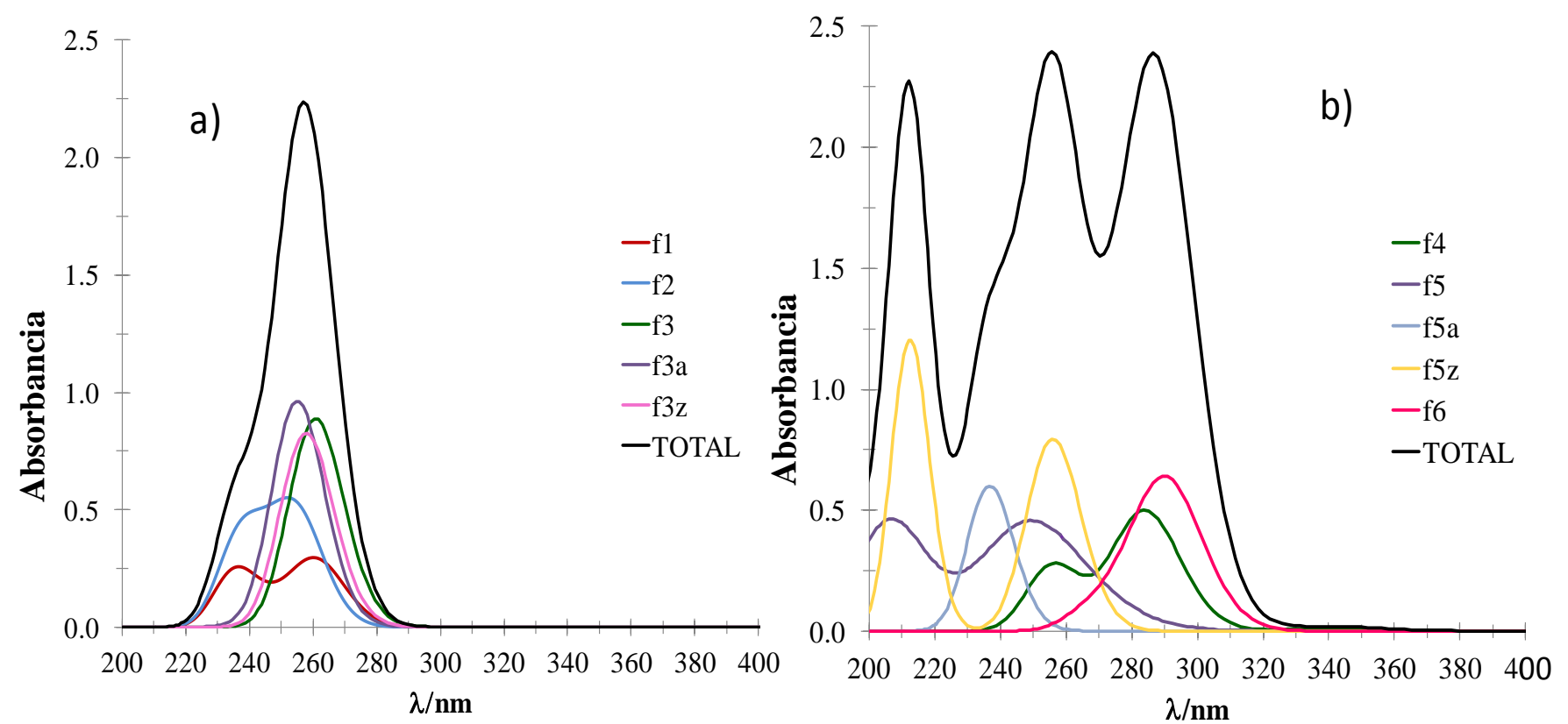

Figura 3.4. Espectros calculados UV-Vis correspondiente a cada uno de los fragmento considerado en a) el esquema II y b) el esquema III. En ambas figuras la etiqueta espectro TOTAL es el resultante de la adición de la absorbancia y la abundancia relativa de los fragmentos formados en cada esquema.

En la figura 3.5 se hace evidente que el espectro total calculado para los fragmentos considerados en el Esquema III representan mucho mejor la evidencia experimental que el calculado para los fragmentos propuestos por Ichihara y colaboradores ${ }^{[3.5,3.7]}$ 

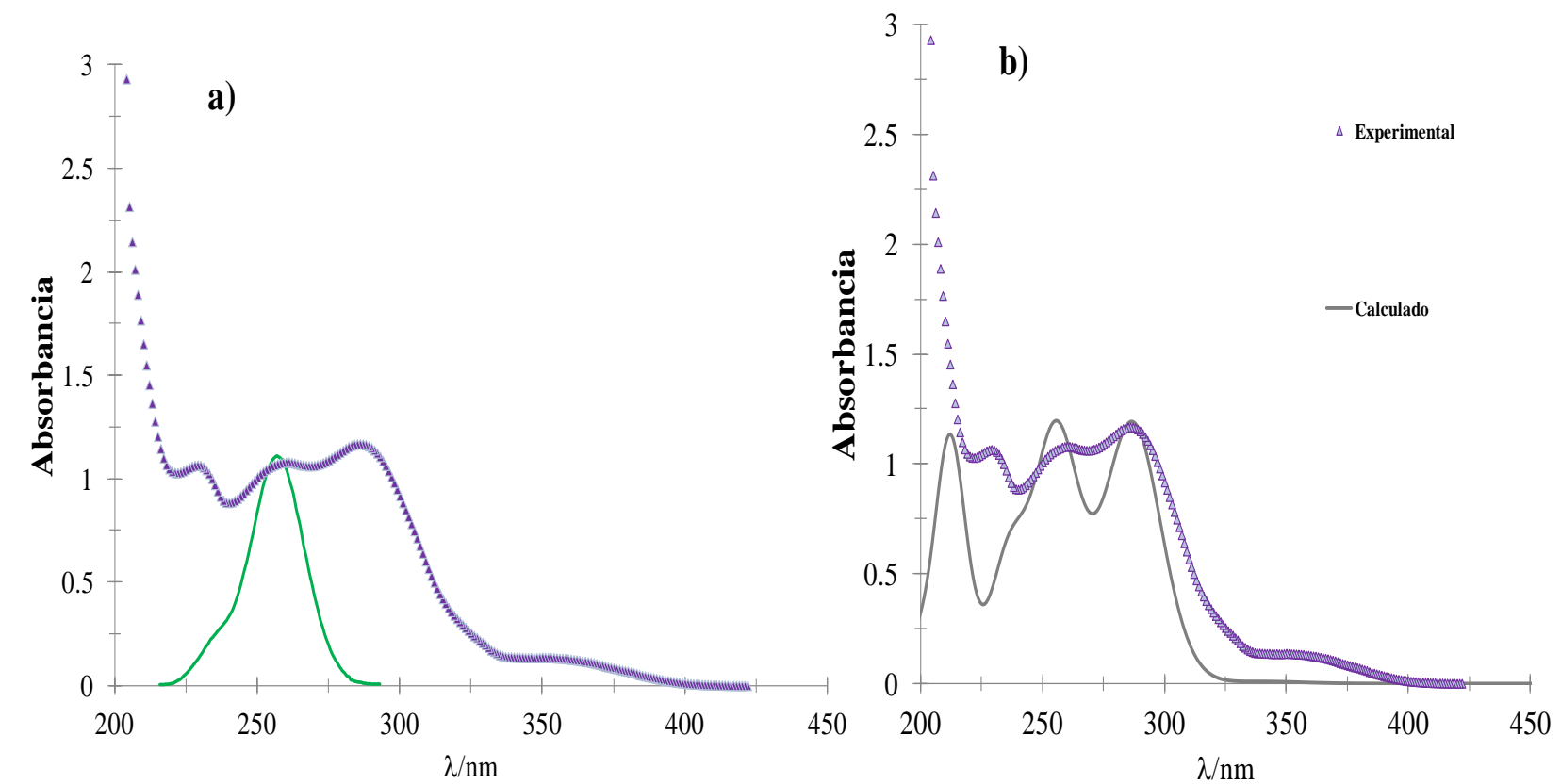

Figura 3.5. Comparación de espectros UV-Vis Total calculados (líneas) de los esquemas a)

3.II y b) 3.III con el espectro obtenido experimentalmente (puntos) después de la imposición de 24 minutos de macroelectrolysis, ver Figura 3.2.

3.4 Estudio de la oxidación de la molécula de Tenoxicam mediante cromatografía líquida de alta presión.

En la figura 3.6 se muestran los cromatogramas experimentales obtenidos después de imponer diferentes tiempos de oxidación en la macroelectrolisis para Tenox en solución acuosa a pH 0.461. Es posible observar que antes de la macroelectrolisis, es decir al tiempo cero, el único producto que puede ser detectado es el correspondiente al Tenox con un tiempo de retención en 4.25 minutos. Después de realizar la oxidación durante 14 y 36 minutos, la intensidad del pico de Tenox disminuye y se resuelven claramente dos picos nuevos con tiempos de retención en aproximadamente 2.8 y 3.6 minutos. 


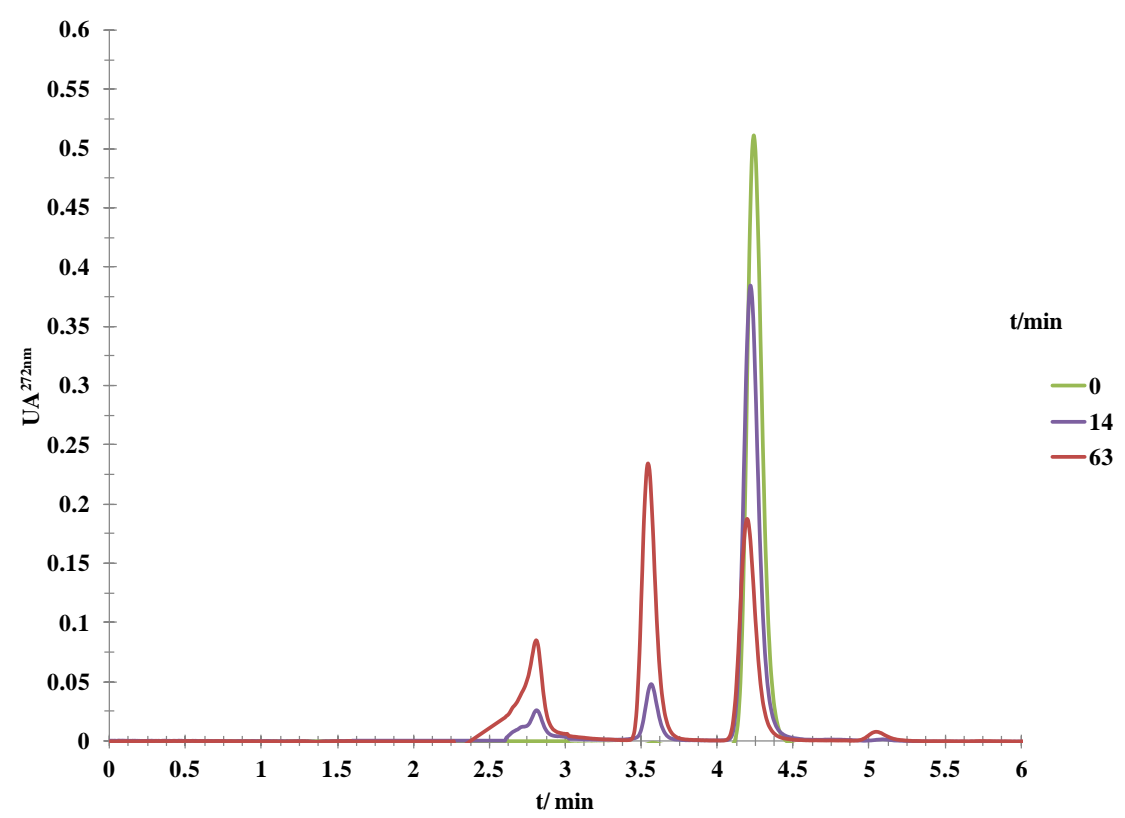

Figura 3.6. Cromatogramas experimental obtenido en solución acuosa con (153 \pm 1$)$ M Tenox después de aplicar diferentes tiempos de oxidación mostrados en la figura a un potencial de $1.0 \mathrm{~V}$, a una longitud de onda constante $\lambda=272 \mathrm{~nm}$. Condiciones experimentales de la sección 3.2.5.

Considerando que el cromatógrafo cuenta con un detector de arreglo de diodos es posible obtener el espectro UV-Vis para cada pico obtenido en el cromatograma, dando pauta a la comparación de los espectros calculados para cada fragmento del esquema 3.III con los obtenidos a partir del cromatograma (ver figura 3.7). 

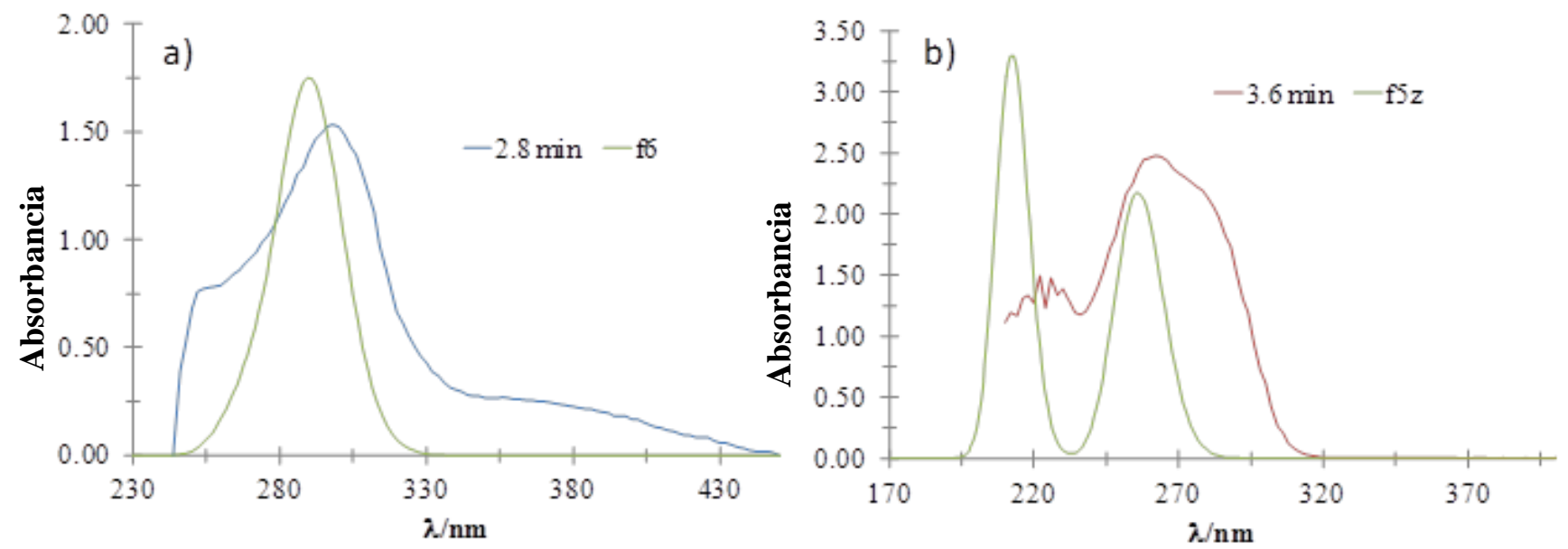

Figura 3.7. Comparación de los espectros UV-Vis calculados para los fragmentos a) f6 y b) f5z con los obtenidos experimentalmente a partir del cromatograma para los tiempos de retención de 2.8 y 3.6 minutos.

En la figura 3.7 es posible observar que los fragmentos que concuerdan con los espectros de absorción experimentales son el fragmento f6 y el fragmento f5z, por lo que pueden considerarse los fragmentos principales del mecanismo de oxidación del Tenox. En la figura 3.8 se presenta el cromatograma del Tenox a diferentes tiempos de oxidación con los fragmentos asociados para cada pico obtenido. 


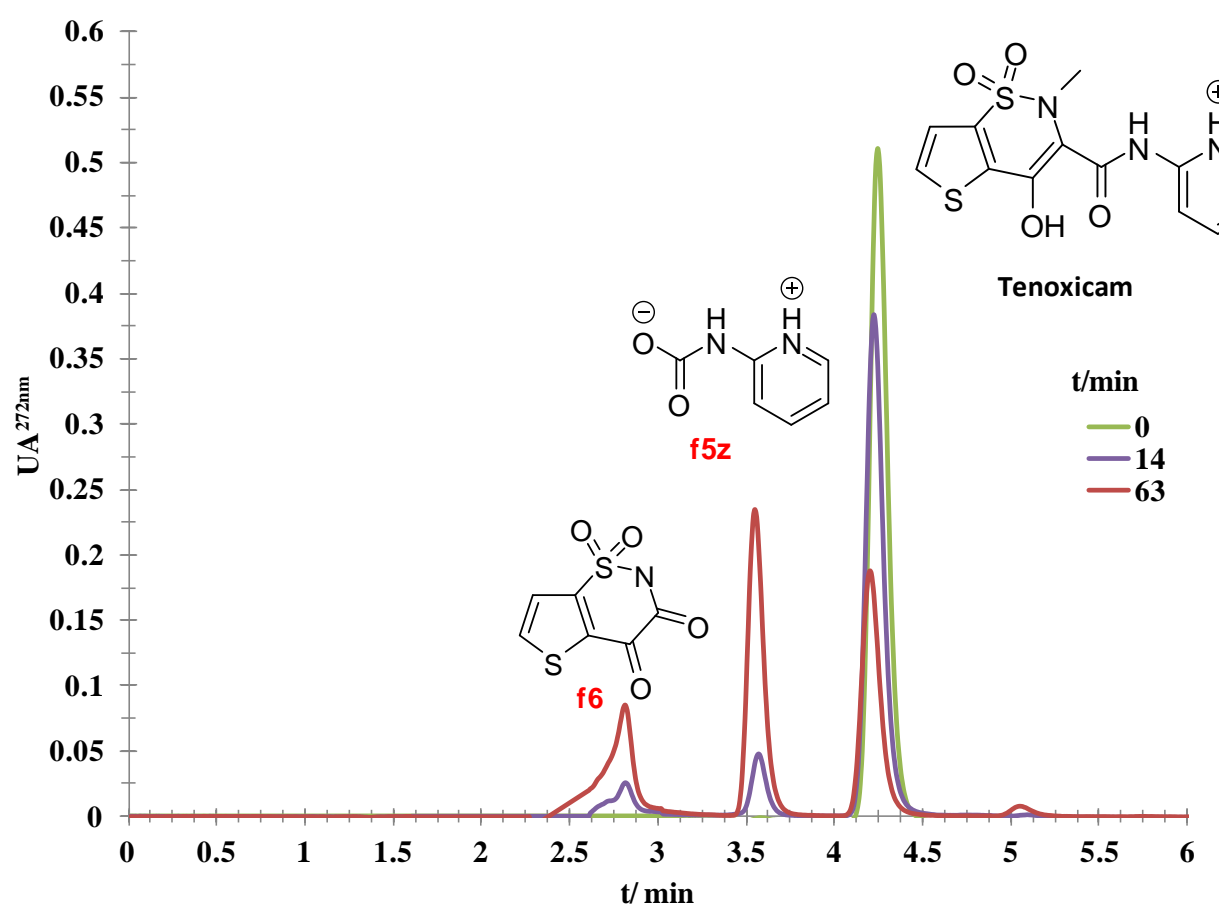

Figura 3.8. Cromatogramas experimental con los fragmentos asociados a cada pico mostrados en la figura, obtenido en solución acuosa con $(153 \pm 1)$ M Tenox después de aplicar diferentes tiempos de oxidación mostrados en la figura a un potencial de $1.0 \mathrm{~V}$. A una longitud de onda constante $\lambda=272 \mathrm{~nm}$.

\subsection{Conclusiones.}

Se determinó que la molécula de $\mathrm{H}_{2} \mathrm{Tenox}^{+}$se rompe dando lugar a un proceso electroquímico químico lo que lo hace completamente irreversible y con ayuda de técnicas experimentales acopladas y cálculos de química cuántica se determinaron los fragmentos que predominan mayoritariamente, dando lugar al fragmento f6 $\mathrm{y}$ f5z descritos en este capítulo. 


\section{CAPÍTULO 4. FORMACIÓN DE COMPLEJOS SUPRAMOLECULARES.}




\section{CAPÍTULO 4. FORMACIÓN DE COMPLEJOS SUPRAMOLECULARES.}

4.1 Introducción.

Como se ha descrito anteriormente, debido a las caracterisiticas de las ciclodextrinas pueden usarse para formar supramoleculas, ya que permiten la formación de complejos de inclusión ${ }^{[4.1]}$. En este capítulo se desarrolla el estudio de la caracterización de la interacción del Tenox con las moléculas de $\beta C D$ utilizando técnicas electroquímicas y espectrofotométricas con el fin de proporcionar nuevas perspectivas en relación al Tenox en solución acuosa.

4.2 Metodología para la formación de complejos de inclusión de Tenoxciam con $\beta$ CD.

\subsubsection{Reactivos.}

La solución de Tenox fue preparada a partir del reactivo grado A R Merck, se trabajó con una solución de $\beta-\mathrm{CD}$ (Fluka 99\%). Para ajustar el pH se utilizó $\mathrm{NaOH}$ (Merck 99\%) y $\mathrm{HCl} /($ Merck 37\%). Todas las soluciones fueron preparadas usando agua desionizada tipo $1(18.2 \mathrm{M} \Omega \mathrm{cm})$ libre de materia orgánica, obtenida de US Filter PURELAB Plus UV cual fue burbujeada con nitrógeno comprimido (Praxair) (1 min/mL).

4.2.1.1 Equipo utilizado para los experimentos electroquímicos.

Se utilizó una celda convencional de tres electrodos, como electrodo de trabajo se utilizó un electrodo de pasta de carbón (EPC) el cual fue preparado a partir de polvo de Grafito (Johnson Matthey $1 \mu \mathrm{m}, 99.9 \%)^{[4.10,4.11]}$. Como contra-electrodo se utilizó un alambre de platino (BAS MW-1032) y como electrodo de referencia se utilizó un electrodo $\mathrm{Ag} / \mathrm{AgCl}$ saturado (BAS MF-2052). Los experimentos electroquímicos se llevaron a cabo con un potenciostato (EPSILON-Bass-i).

Vis.

4.2.1.2 Equipo utilizado para los experimentos espectrofotométricos UV-

Los estudios espectrofotométricos se llevaron a cabo utilizando un espectrofotómetro UV-Vis Perkin Elmer Lambda 20 y una celda de cuarzo de $1 \mathrm{~cm}$ de longitud de paso óptico. 
El pH de las soluciones fue medido usando un potenciómetro Mettler Lab pHM240 con una resolución de 0.001 de $\mathrm{pH}$ con un electrodo combinado de vidrio de intervalo de pH $\quad 0-14$ ((Mettler Toledo InLab® Expert NTC30 + DIN/RCA)). Durante la experimentación se controló la temperatura a $25^{\circ} \mathrm{C}$ y se mantuvo la solución en atmósfera inerte de nitrógeno.

\subsection{Estudio de la formación de complejos Tenoxicam - $\beta$-CD.}

Para la formación del complejo de Tenox con $\beta$-CD se considera la reacción 4.1 la cual muestra el equilibrio general para las especies de Tenox con $\beta$-CD.

$$
\text { Tenox }+C D^{\prime} \Leftrightarrow \text { Tenox } C D^{\prime} \ldots \ldots . . . .(4.1)
$$

La constante de formación global para el equilibrio químico 4.1 está dada por

$$
\beta^{\prime}=\frac{\mid \text { Tenox } C D^{\prime} \mid}{\mid \text { Tenox }^{\prime}\left|C D^{\prime}\right|}
$$

Donde Tenox' representa todas las especies de Tenox y $C D^{\prime}$ representa la $\beta-C D$.

$$
\text { Tenox }^{\prime}=\mathrm{H}_{2} \text { Tenox }^{+}+\text {HTenox }^{+} \text {Tenox }^{-} \text {. }
$$

De tal manera que a un $\mathrm{pH}$ constante y en función de $C D^{\prime}$ es posible tener un Tenox' ${ }_{\text {Total }}$

$$
\text { Tenox }_{\text {Total }}^{\prime}=\text { Tenox }^{\prime}+\text { Tenox } C D^{\prime} \text {..........(4.3) }
$$

De la constante de formación global del equilibrio 4.1 se despeja TenoxCD' y se sustituye en la ecuación 4.3.

$$
\text { Tenox }_{\text {Total }}^{\prime}=\mid \text { Tenox }^{\prime}\left(1+\beta^{\prime}\left|C D^{\prime}\right|\right) \ldots \ldots . . .(4.4)
$$

Ahora bien, la fracción molar de la especie del Tenox' se define como se muestra en la ecuación 4.5 .

$$
f_{\text {Tenox }^{\prime}}=\frac{\mid \text { Tenox }^{\prime} \mid}{\mid \text { Tenox }_{\text {Total }}^{\prime} \mid} \ldots \ldots . . . . .(4.5)
$$

Sustituyendo el valor de Tenox $_{\text {Total }}^{\prime}$ de la ecuación 4.4 en 4.5 se tiene la fracción molar para la especie de Tenox'. 


$$
f_{\text {Tenox' }}=\frac{1}{1+\beta^{\prime}\left|C D^{\prime}\right|} \ldots \ldots \ldots . .(4.6)
$$

De igual manera se define la fracción molar para la especie de TenoxCD' como se muestra en la ecuación 4.7.

$$
f_{\text {TenoxCD }}=\frac{\mid \text { TenoxCD }^{\prime} \mid}{\mid \text { Tenox }_{\text {Total }}^{\prime} \mid} \ldots \ldots \ldots . . .(4.7)
$$

Sustituyendo nuevamente el valor de Tenox $_{\text {Total }}$ de la ecuación 4.4 y el valor despejado de Tenox $C D^{\prime}$ en la ecuación 4.7 se tiene la fracción molar para la especie de TenoxCD'.

$$
f_{\text {Tenox }}=\frac{\left|C D^{\prime}\right| \beta^{\prime}}{\left(1+\beta^{\prime}\left|C D^{\prime}\right|\right)} \ldots \ldots \ldots . . .(4.8)
$$

La suma de la fracciones de las especies debe ser igual a 1 como se muestra en la ecuación 4.9.

$$
f_{\text {Tenox }}+f_{\text {TenoxCD }}=1 \text {.........(4.9) }
$$

Con las fracciones molares para cada especie y a un $\mathrm{pH}$ constante es posible obtener un gráfico donde se observa el predominio de cada especie de Tenox' ${ }_{\text {Total }}$ en función de la concentración de $\beta$-CD y $\mathrm{pH}$.

Ahora bien, es posible seguir una propiedad física en función de las especies de Tenox' ; esta propiedad física puede ser la absorbancia la cual sigue la ley de Beer-Lambert Siguiendo esta ley es posible determinar la absorbacia para cada especie de Tenox' como se muestra en la ecuación 4.10 .

$$
\begin{aligned}
& A_{\text {Tenox' }}^{\lambda_{1}}=\varepsilon_{\text {Tenox }}^{\lambda_{1}} l \mid \text { Tenox } \\
& A_{\text {TenoxCD }}^{\lambda_{1}}=\varepsilon_{\text {TenoxCD }}^{\lambda_{1}} \mid \text { TenoxCD }
\end{aligned}
$$

Donde $\boldsymbol{A}^{\lambda_{1}}$ es la absorbancia de la especie a una longitud de onda dada, $\varepsilon^{\lambda_{1}}$ es el coeficiente de absortividad de la especie a una longitud de onda dada, $l$ es la longitud de paso óptico de la celda y $\mid$ Tenox' $\mid$ es la concentración de las especies de Tenox.

La absorbancia total será igual a la suma de las absorbancias para cada especie como se muestra en la ecuación 4.11. 


$$
A_{\text {Total }}^{\lambda_{1}}=A_{\text {Tenox' }}^{\lambda_{1}}+A_{\text {TenoxCD }}^{\lambda_{1}} \cdots \cdots . . .(4.11)
$$

La intensidad de corriente es otra propiedad física que se puede seguir en función de las especies de Tenox' permitiendo determinar la formación del complejo. Así entonces el estudio de formación del complejo de inclusión de Tenox con $\beta C D$, se lleva a cabo a diferentes valores de $\mathrm{pH}$ para asegurar el predominio de cada una de las especies de Tenox $\operatorname{con} \beta-\mathrm{CD}$.

4.3.1 Determinación espectrofotométrica de la constante de inclusión del $\mathrm{H}_{2}$ Tenox $^{+} \operatorname{con} \beta-\mathrm{CD}$ a $\mathrm{pH} 0.410$

En la Figura 4.1 se muestran los espectros de absorción experimentales de Tenox obtenidos a $\mathrm{pH} 0.410$ y diferentes concentraciones de $\beta$-CD. Estos espectros presentan un máximo de absorción en 354 nm. Es posible notar un efecto hipocrómico en el máximo de absorción conforme se aumenta la concentración de $\beta-\mathrm{CD}$, este efecto se asocia a la disolución del Tenox en la solución.

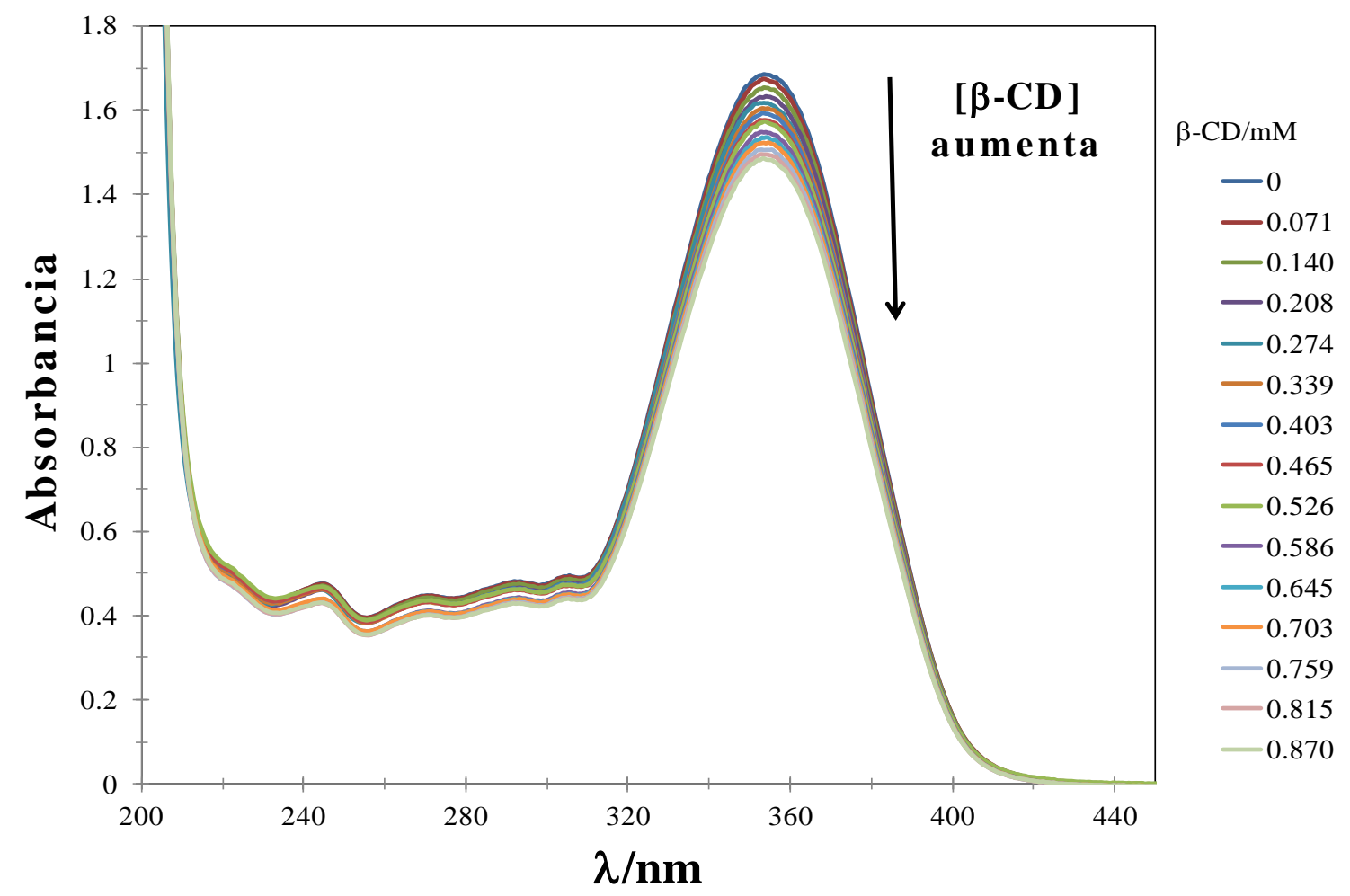


Figura 4.1. Espectros de absorción obtenidos en una disolución $30.10 \mu \mathrm{M}$ de Tenox $\left(\mathrm{H}_{2} \mathrm{Tenox}^{+}\right)$a $\mathrm{pH} 0.410$ y diferentes valores de concentraciones de $\beta-\mathrm{CD}$, indicadas en la figura en $\mathrm{mM}$.

Para calcular la constante de formación del Tenox con $\beta$-CD se utilizó el programa computacional SQUAD ${ }^{[4.3]}$, el cual se alimenta con los espectros de absorción experimentales, la concentración del Tenox, la concentración de $\beta-\mathrm{CD}$, el valor de $\mathrm{pH}$ y la propuesta del equilibrio R1.

$$
\mathrm{H}_{2} \mathrm{Tenox}^{+}+\beta-\mathrm{CD} \rightleftharpoons \beta-\mathrm{CD}_{-} \mathrm{H}_{2} \mathrm{Tenox}^{+} \quad \mathrm{R} 1
$$

Del programa SQUAD se obtienen los coeficientes de absortividad molar a cada longitud de onda así como también el valor para la constante de formación, LogK para el equilibrio propuesto R1, junto con su desviación estándar (DS) y la suma de mínimos cuadrados (U), los cuales se muestran en la Tabla 4.1.

Tabla 4.1. Constante de formación obtenida a partir de los espectros mostrados en la Figura 3 y del programa SQUAD, para 50 espectros de absorción y un intervalo de longitud de onda $220 \mathrm{~nm}$ a $500 \mathrm{~nm}$

\begin{tabular}{|l|l|}
$\mathrm{H}_{2} \mathrm{Tenox}^{+}+\beta-\mathrm{CD} \rightleftharpoons \beta-\mathrm{CD}_{-} \mathrm{H}_{2} \mathrm{Tenox}^{+}$ & $\operatorname{LogK}=2.982 \pm 0.005$
\end{tabular}

$\mathrm{DS}=0.0022 \mathrm{U}=0.022$

En la Figura 4.2 se muestran los coeficientes de absortividad molar con sus desviaciones estándar respectivas dadas por SQUAD para la especie de Tenox involucrada en el equilibrio R1. 


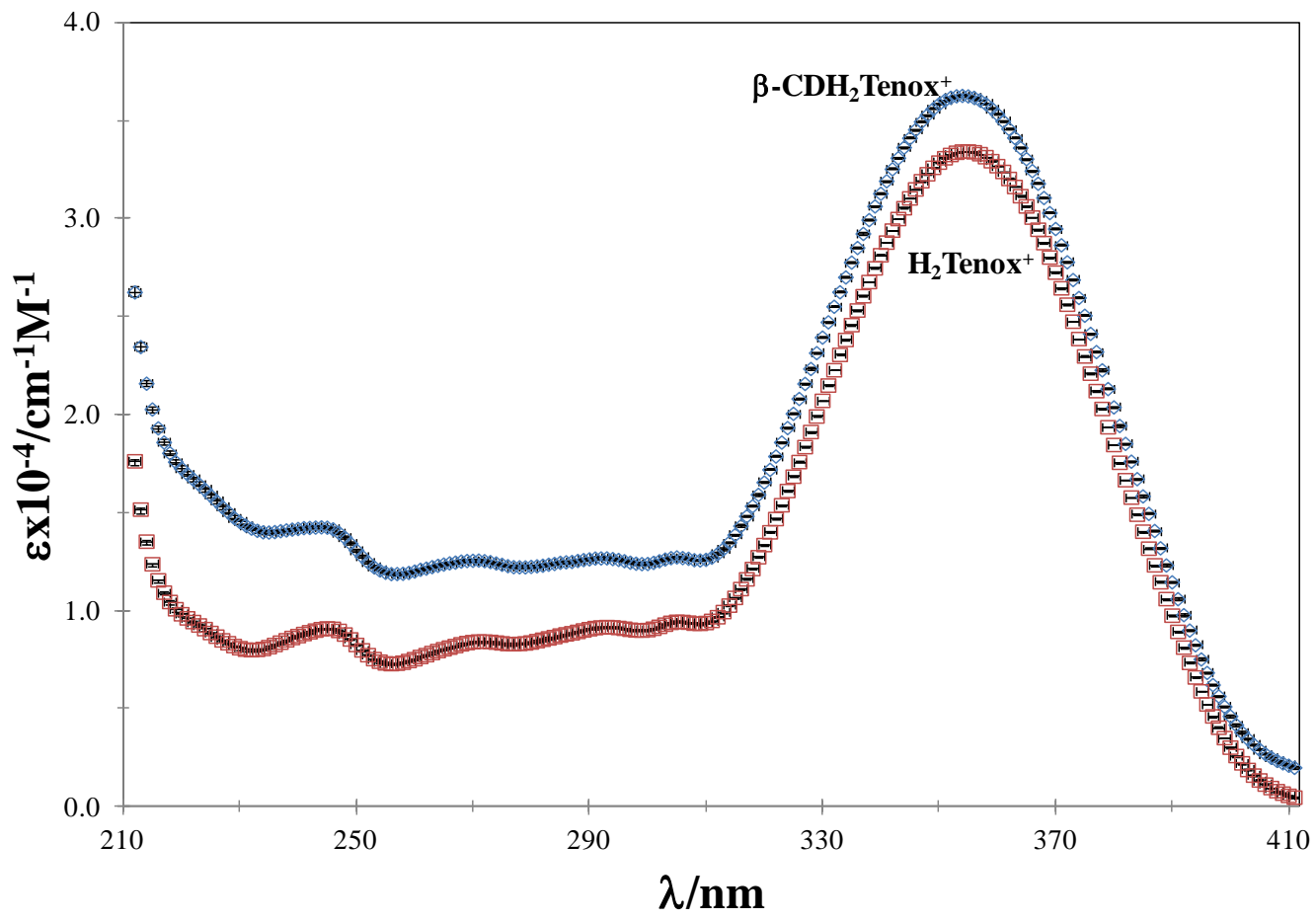

Figura 4.2. Variación del coeficiente de absortividad molar en función de la longitud de onda obtenido a partir de SQUAD para las especies de Tenox involucradas en el equilibrio R1.

A partir de los datos obtenidos por SQUAD es posible realizar un ajuste de los espectros de absorción experimentales con los espectros calculados? ${ }^{\text {l }}$ En la Figura 4.3 se muestra la comparación de dos espectros de absorción obtenidos experimentalmente a p$\beta C D$ de 2.797 para una concentración de Tenox de $30.04 \mu \mathrm{M}$ y p- $\beta C D 5.161$ para una concentración de Tenox de $26.47 \mu \mathrm{M}$ con los calculados mediante SQUAD donde se puede observar que los espectros de absorción calculados reproducen la información obtenida experimentalmente. 


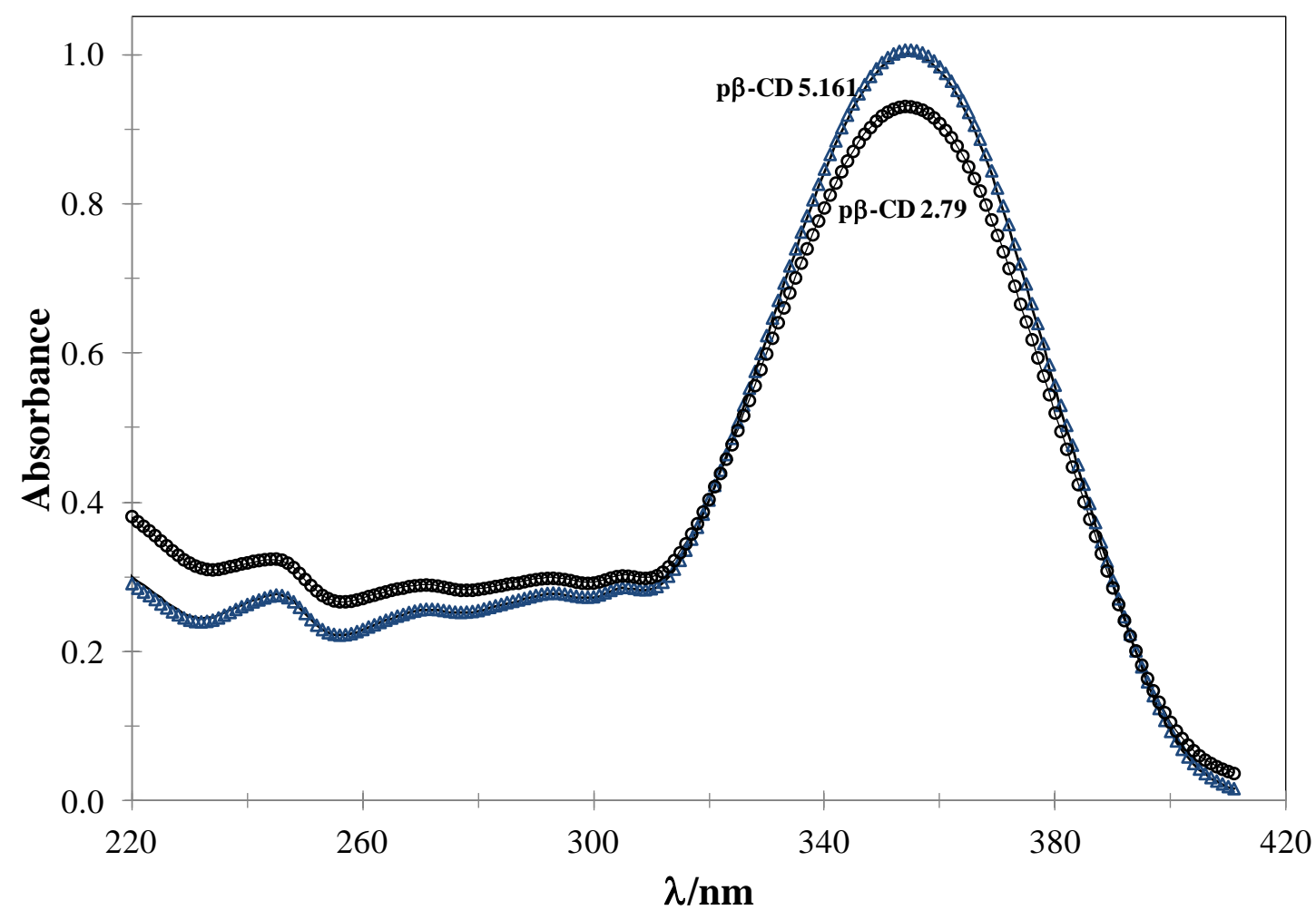

Figura 4.3. Comparación de espectros de absorción obtenidos experimentalmente pH 0.410 (puntos) para dos valores de $\mathrm{p} \beta \mathrm{CD}(\triangle) 4.151$ y $(\bigcirc) 2.131$ y los espectros de absorción calculados mediante el programa SQUAD (líneas).

4.3.2 Determinación espectrofotométrica de la constante de inclusión del HTenox(z) (pH $3.175)$ y Tenox - $(\mathrm{pH} 7.150)$ con $\beta-\mathrm{CD}$

En la Figura 4.4 se muestran los espectros de absorción experimentales obtenidos en una disolución de Tenox y diferentes concentraciones de $\beta$-CD para dos valores de $\mathrm{pH}$; 3.325 (Figura 4.4a) y 7.010 (Figura 4.4b). En ambos casos se puede notar un pico de máximo de absorción en 374 nm, para pH 3.325, y en 360 nm para pH 7.010, cuyo valor de absorción disminuye conforme aumenta la concentración de $\beta$-CD en la disolución. De igual manera que en el estudio a pH 0.410 este efecto se asocia a la disolución del Tenox en la solución. 

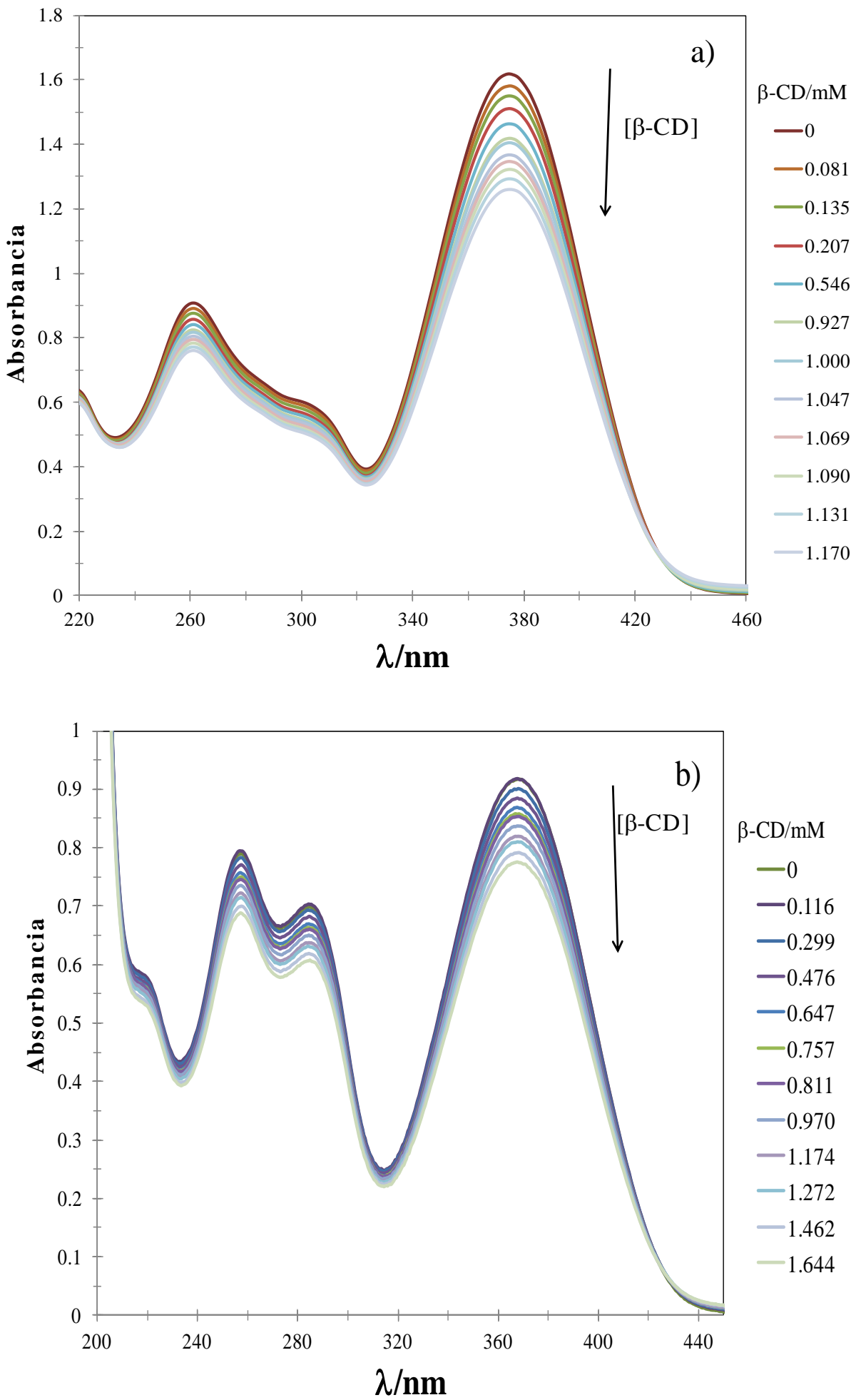

Figura 4.4. Espectros de absorción experimentales obtenidos en una disolución de Tenox $30.10 \mu \mathrm{M}$ y diferentes concentraciones de $\beta$-CD indicados en la figura para dos valores de $\mathrm{pH}$; (a) 3.325 (HTenox(z)) y (b) 7.010 (Tenox $^{-}$). 
Al realizar el cálculo de la constante de formación para las especies de $\operatorname{HTenox}(z)$ y de Tenox ${ }^{-}$con $\beta$-CD mediante el programa computacional SQUAD, se propusieron los siguientes equilibrios:

$$
\begin{gathered}
\text { Para pH 3.325 } \\
\text { HTenox }(z)+\beta-C D \rightleftharpoons \beta-C D-H \operatorname{Henox}(z) \quad \mathrm{R} 2
\end{gathered}
$$$$
\text { Para pH } 7.010
$$$$
\text { Tenox }^{-}+\beta-C D \rightleftharpoons \beta-C D-\text { Tenox }^{-}
$$

A partir de los datos especificados para SQUAD, el programa convergió a los valores que se muestran en la Tabla 4.2. En esta misma tabla se muestra la comparación de LogK obtenidos para los diferentes $\mathrm{pH}$ impuestos.

Tabla 4.2. Constantes de formación, DS y U para los equilibrios R2 y R3, para 50 espectros de absorción y un intervalo de longitud de onda 220nm a 500nm.

\begin{tabular}{|c|l|c|c|c|}
\hline $\mathrm{pH}$ & \multicolumn{1}{|c|}{ Equilibrio } & $\operatorname{LogK}$ & $\mathrm{SD}$ & $\mathrm{U}$ \\
\hline 0.410 & $\mathrm{H}_{2}$ Tenox $^{+}+\beta-\mathrm{CD} \rightleftharpoons \beta-\mathrm{CD}^{-\mathrm{H}_{2} \text { Tenox }^{+}}$ & $2.982 \pm 0.005$ & 0.022 & 0.022 \\
\hline 3.325 & HTenox $\mathrm{z})+\beta-\mathrm{CD} \rightleftharpoons \beta-$ CD-HTenox $(z)$ & $3.705 \pm 0.005$ & 0.002 & 0.026 \\
\hline 7.010 & Tenox $^{-}+\beta-\mathrm{CD} \rightleftharpoons \beta-$ CD-Tenox $^{-}$ & $3.285 \pm 0.017$ & 0.002 & 0.007 \\
\hline
\end{tabular}



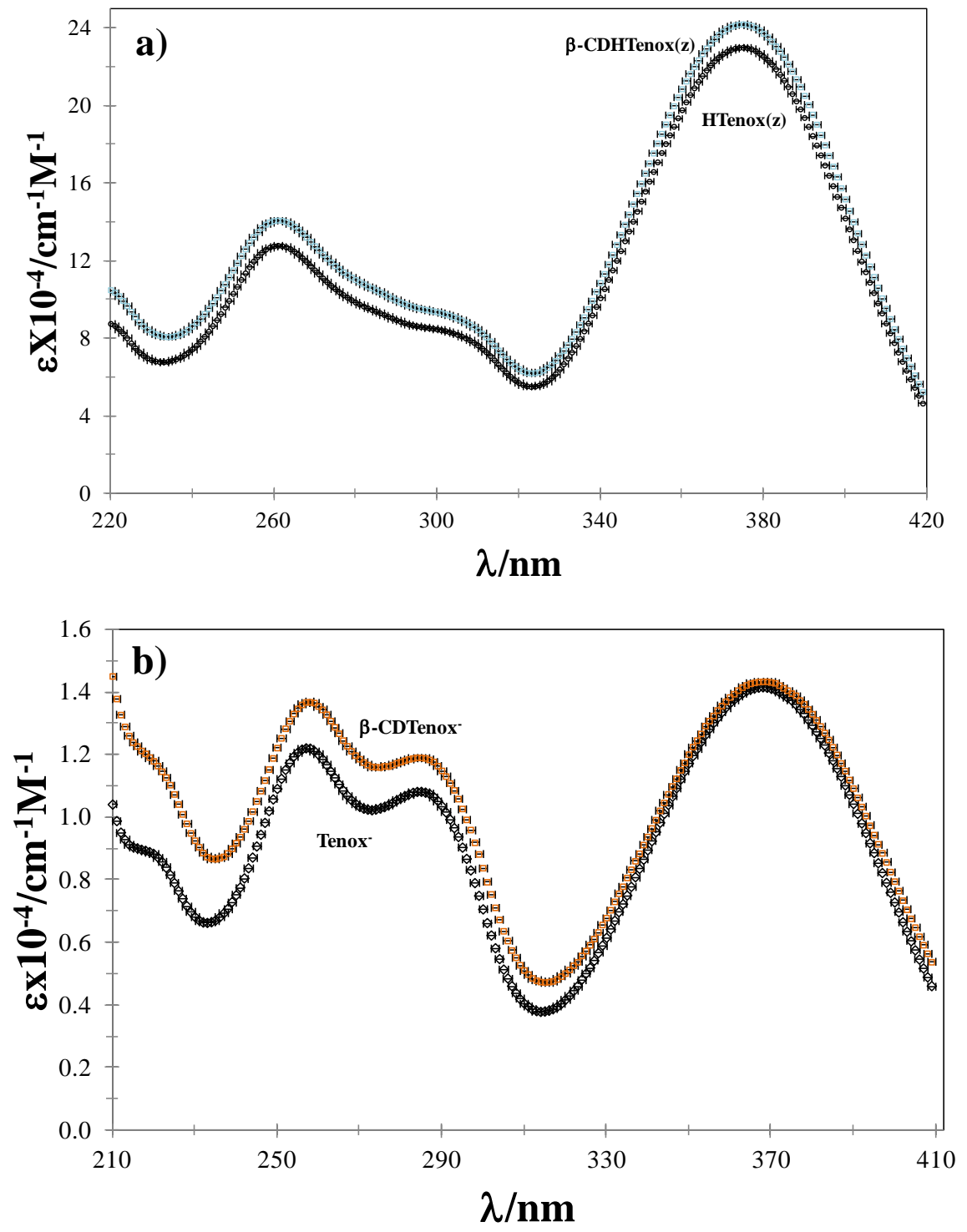

Figura 4.5. Variación de los coeficientes de absortividad molar en función de la longitud de onda obtenido a partir del programa SQUAD para las especies de Tenox involucradas en los equilibrios R2 (a) y R3 (b).

En la Figura 4.5 se muestran los coeficientes de absortividad molar con sus desviaciones estándar respectivas dadas por el programa SQUAD para las especies de Tenox involucradas en los equilibrios R2 (Figura 4.5a) y R3 (Figura 4.5b).

En la Figura 4.6 se muestra la comparación de espectros de absorción obtenidos experimentalmente para diferentes valores de $\mathrm{pH} 3.325$ (Figura 4.6a) y 7.150 (Figura 4.6b) y con los calculados mediante el programa SQUAD. Como se puede observar en ambos 
casos los espectros de absorción calculados reproducen a los espectros de absorción experimentales.
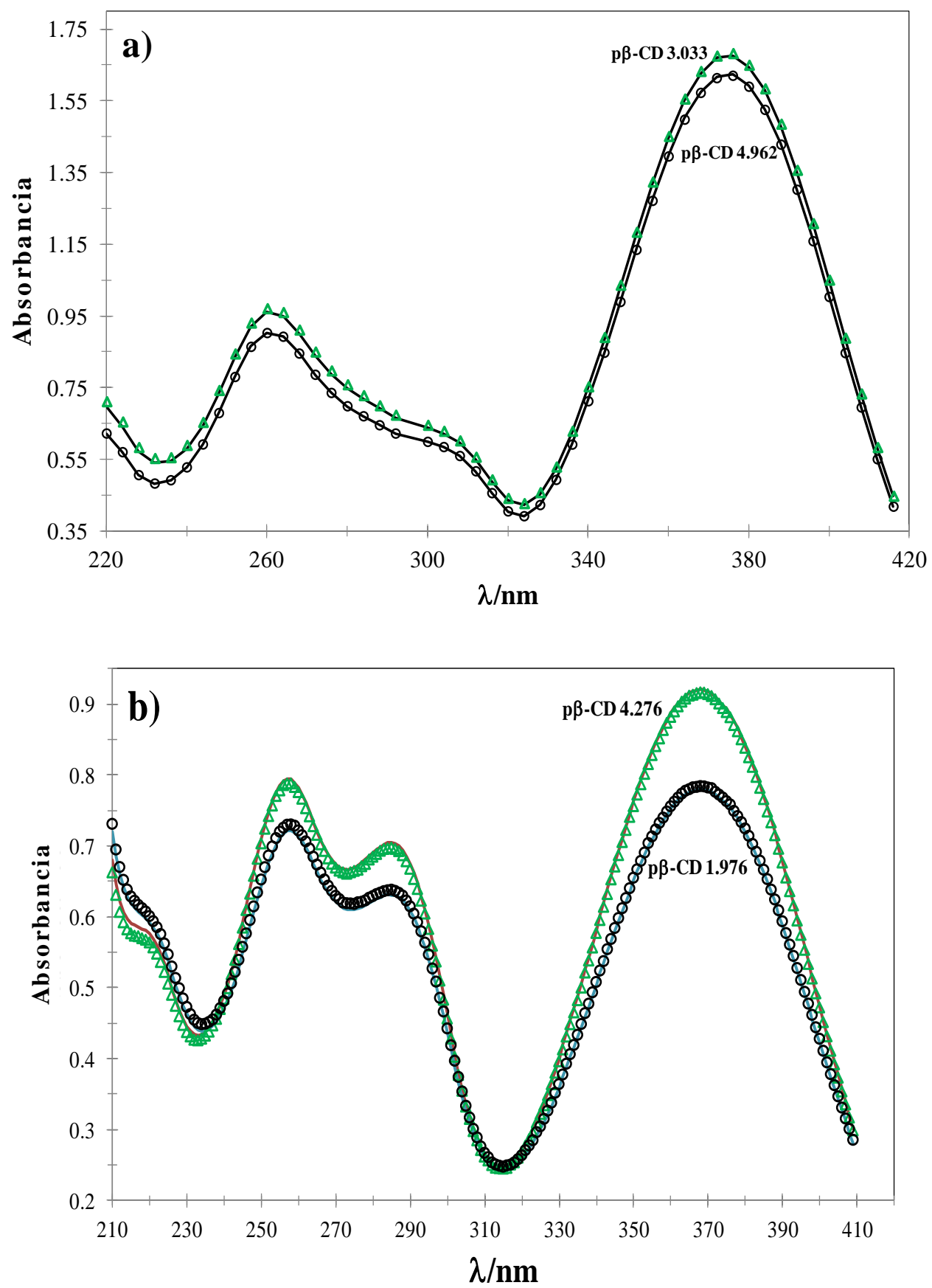

Figura 4.6. Comparación de los espectros de absorción experimentales (puntos) obtenidos a diferentes valores de $\mathrm{pH}$ (a) 3.325 y (b) 7.150 y dos valores de $\mathrm{p} \beta-\mathrm{CD}$, indicados en la figura, con los espectros de absorción calculados (líneas) mediante el programa SQUAD. 
A partir de las constantes de formación global de acidez de Tenox (ver Tabla 1) y las constantes de formación $(\operatorname{LogK})$ de los complejos de inclusión, ver Tablas 2 y 3, es posible obtener los valores de las constantes de formación global de los complejos de inclusión ( $\beta$ ), ver Tabla 2.3.

Tabla 2.3. Constantes de formación global de los complejos de inclusión entre el Tenox y la $\beta-\mathrm{CD}$.

\begin{tabular}{|c|c|}
\hline Equilibrio global & $\log \beta$ \\
\hline Tenox $^{-}+2 \mathrm{H}^{+}+\beta-\mathrm{CD} \rightleftharpoons \quad \beta-\mathrm{CD}-\mathrm{H}_{2}$ Tenox $^{+}$ & $9.198 \pm 0.004$ \\
\hline Tenox $^{-}+H^{+}+\beta-C D \rightleftharpoons \beta-C D-H T \operatorname{Tenox}(z)$ & $8.847 \pm 0.005$ \\
\hline Tenox $^{-}+\beta-C D \rightleftharpoons \beta-C D-$ enox $^{-}$ & $3.285 \pm 0.017$ \\
\hline
\end{tabular}

Utilizando el programa computacional MEDUSA ${ }^{[4.4]}$, las constantes de formación global de acidez de Tenox y las constantes de las Tabla 2.3 es posible determinar la las fracciones molares para cada especie de Tenox, libre y acomplejado. Con esta información se construyeron los diagramas tridimensionales fracción molar- $[\beta-\mathrm{CD}]-\mathrm{pH}$ para las diferentes especies de los complejo de inclusión Tenox- $\beta$-CD (Figura 4.7a), así mismo se presenta un diagrama de fracción molar en función de $\mathrm{pH}$ a un $\mathrm{p} \beta \mathrm{CD}=4.277$ (Figura 4.7b), donde es posible observar el predominio de las especies de Tenox libres de $\beta C D$ y a un $\mathrm{p} \beta C D=2.892$ (Figura 4.7c) el predominio de las especies complejadas Tenox- $\beta$-CD. 

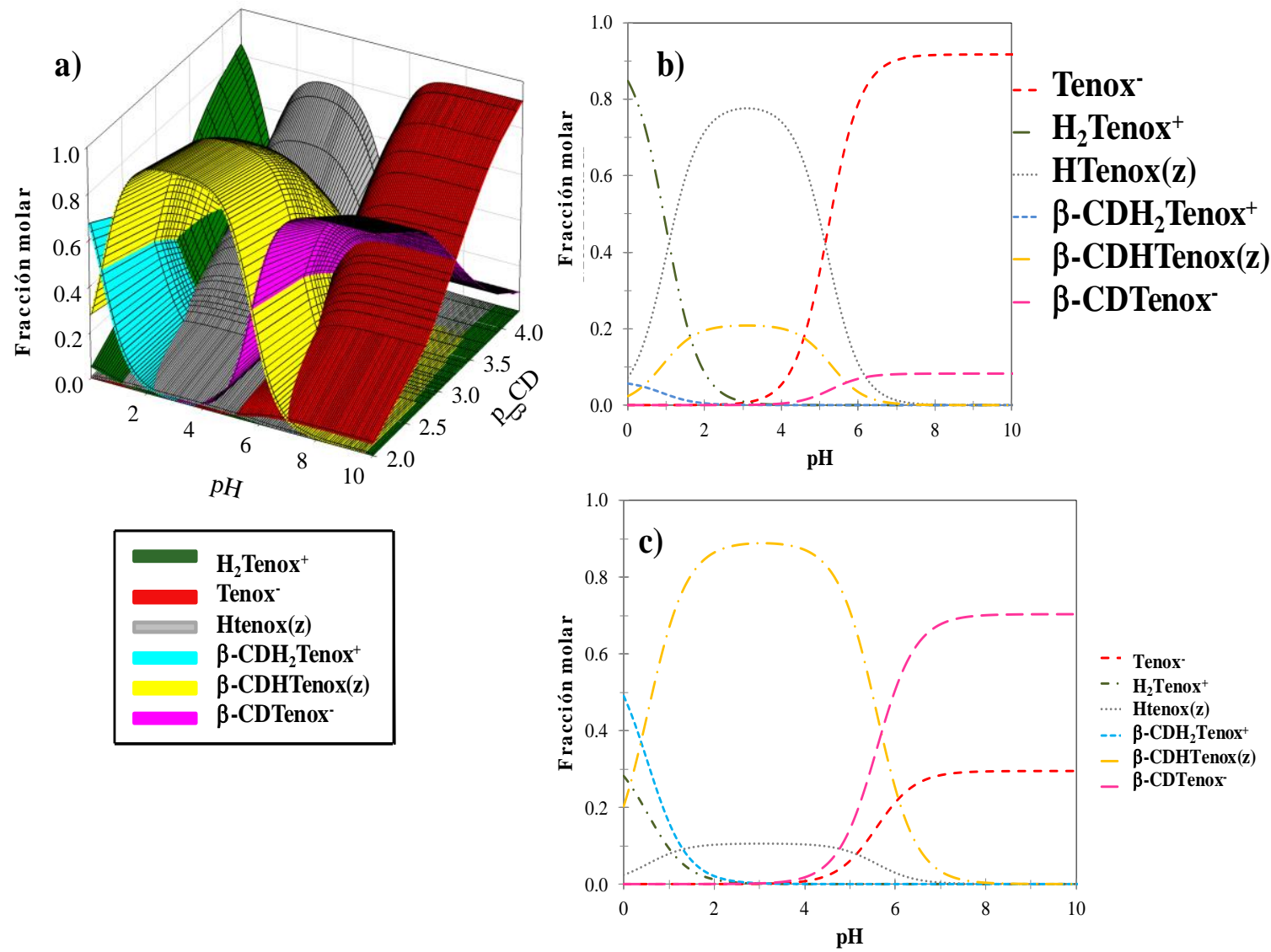

Figura 4.7. (a) Diagramas tridimensionales fracción molar-[ $\beta-\mathrm{CD}]-\mathrm{pH}$ para las diferentes especies de los complejo de inclusión Tenox- $\beta$-CD $\left(\beta-\mathrm{CD}_{-}-\mathrm{Tenox}^{-}, \beta-\mathrm{CD}-\mathrm{HTenox}(\mathrm{z})\right.$ y $\beta-\mathrm{CD}-$ $\mathrm{H}_{2}$ Tenox $^{+}$) . Diagrama de fracción molar en función de $\mathrm{pH}$ a un $\mathrm{p} \beta-\mathrm{CD}$ ( b) 4.277 y (c) 2.892 .

Más aún, a partir de las constantes globales de acidez de Tenox $(\log \beta)$ y las constantes de formación (K) de los complejos de inclusión (ver Tablas 4.1, 4.2 y 4.3) es posible construir el diagrama de zonas de predominio lineal en función de $\mathrm{pH}$ para de los complejo de inclusión Tenox- $\beta-\mathrm{CD}^{[4.4-4.9]}$ (ver Figura 4.8).

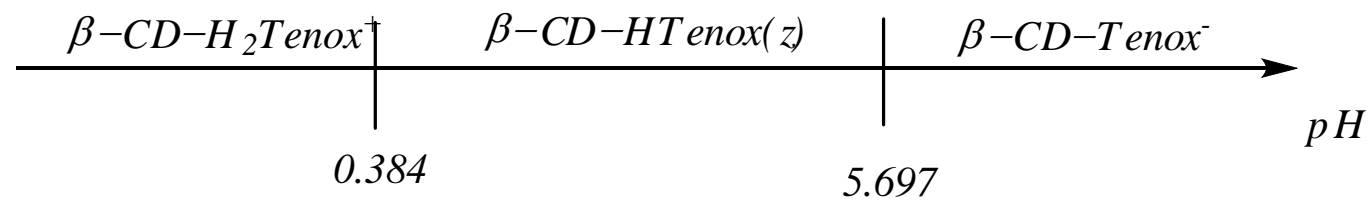

Figura 4.8. Diagrama de zonas de predominio lineal para los complejo de inclusión Tenox- $\beta-\mathrm{CD}$ en función de $\mathrm{pH}$. 
4.3.3 Determinación electroquímica de la constante de inclusión del $\mathrm{H}_{2} \mathrm{Tenox}^{+}$con $\beta-\mathrm{CD}(\mathrm{pH} 0.420)$

Se realizó el estudio electroquímico utilizando voltamperometría cíclica a una solución de Tenox $160 \mu \mathrm{M}$ a un $\mathrm{pH}$ de 0.420 . En todos los casos se observa durante el barrido de potencial en dirección positiva un pico de oxidación, alrededor de $0.78 \mathrm{~V}$. Al invertir el barrido de potencial no se observa ningún pico de reducción debido a que la oxidación electroquímica del Tenox se lleva a cabo mediante un mecanismo EC limitado por la difusión donde la reacción química acoplada conlleva a la ruptura de la molécula de Tenox en dos fragmentos predominantes y con ello a la irreversibilidad de los procesos. Al aumentar la concentración de $\beta-\mathrm{CD}$ en la disolución se presenta un decremento en la corriente de pico de oxidación como se aprecia en el inserto de la Figura 4.9.

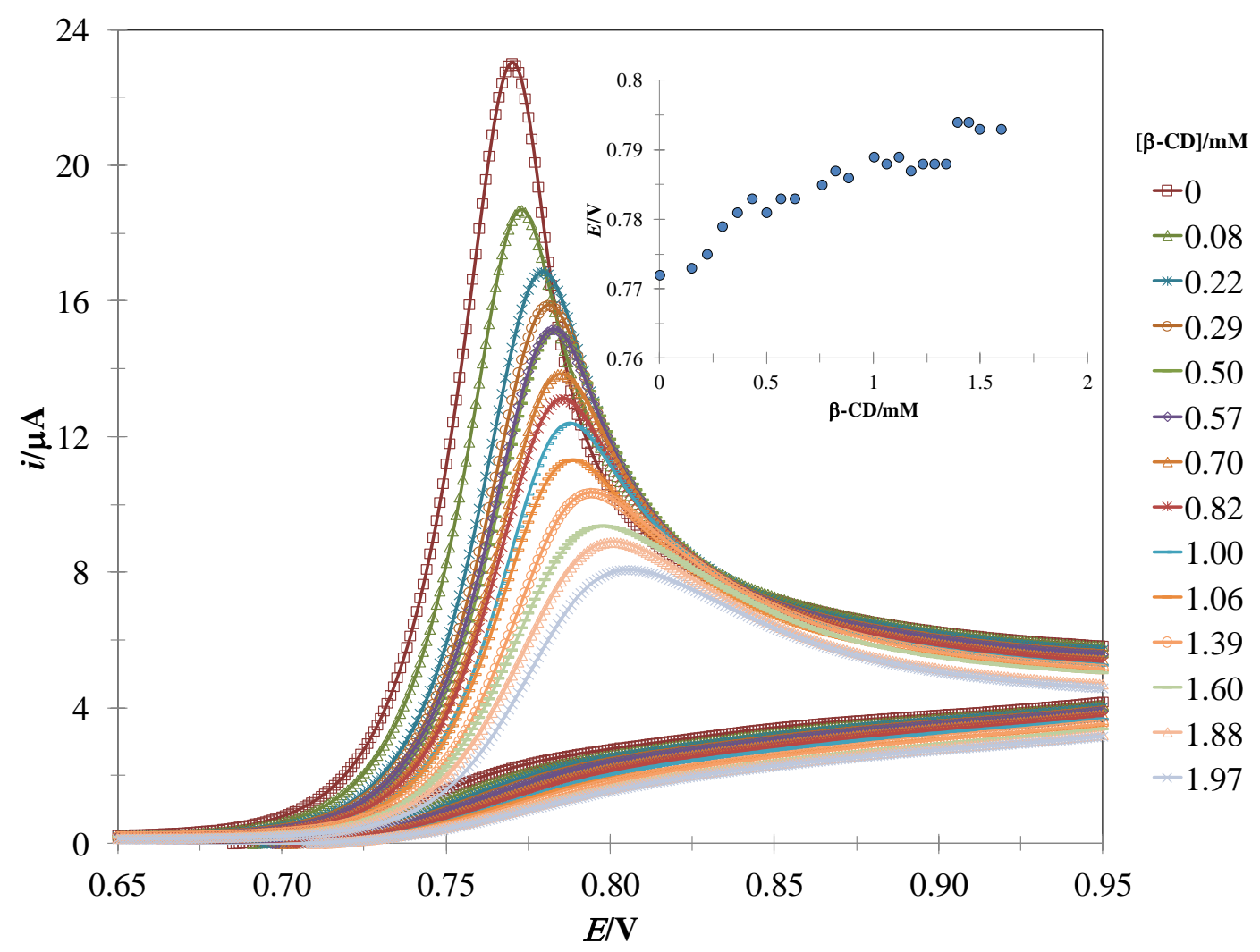

Figura 4.9. Familia de VCs obtenidos en el sistema EPC / $160 \mathrm{mM}$ Tenox, $1 \mathrm{M} \mathrm{Cl}^{-}$ a pH 0.420 con diferentes concentraciones de $\beta-\mathrm{CD}$ mostrados en la Figura. En todos los casos el barrido de potencial comenzó en $0.0 \mathrm{~V}$ en dirección anódica a una velocidad de barrido fue de $0.1 \mathrm{Vs}^{-1}$. El inserto muestra la variación de la corriente de pico anódica en función de la concentración de $\beta-\mathrm{CD}$. 
De acuerdo con Gao y colaboradores ${ }^{[4.10]}$ a partir de la pendiente asociada a la gráfica de la ecuación 4.13 es posible obtener la constante de disociación del complejo de inclusión $K_{\mathrm{d}}$.

$$
i_{p}^{2}=\frac{K_{d}}{[\beta-C D]}\left(i_{p(\text { Tenox })}^{2}-i_{p}^{2}\right)+i_{p(\text { Tenox }-\beta-C D)}^{2}
$$

Donde $[\beta-\mathrm{CD}]$ es la concentración de $\beta-\mathrm{CD}, i_{\mathrm{p}}$ es la corriente de pico medida, $i_{\mathrm{p}(\mathrm{Tenox})}$ es la corriente de pico de Tenox libre (para $\left.[\beta-\mathrm{CD}]=0\right), i_{(\text {Tenox- } \beta C D)}$ es la corriente de pico del complejo Tenox- $\beta \mathrm{CD}$ (para $[\beta C D]>0)$.

Para los datos asociados a la Figura 4.8 la recta de regresión obtenida es la mostrada en la ecuación (4.14).

$$
i_{p}^{2}\left(A^{2}\right)=(9.85 \pm 0.53) \times 10^{-4} M * \frac{\left(i_{p(\text { Tenox })}^{2}-i_{p}^{2}\right)}{[\beta-C D]}+(0.27 \pm 0.62) \times 10^{-4} A^{2}
$$

Por lo tanto a partir del valor de la pendiente se obtiene que para este caso el valor de $\mathrm{K}_{\mathrm{d}}$ del complejo de inclusión $\beta-\mathrm{CDH}_{2} \mathrm{Tenox}^{+}$es $(9.85 \pm 0.53) \times 10^{-4} \mathrm{M}$. Por lo tanto el valor de la constante asociado al equilibrio $\mathrm{R} 1$, obtenido por este método es $-\log K_{d}=$ $3.01 \pm 0.10$

4.3.4 Determinación electroquímica de la constante de inclusión del HTenox $(\mathrm{pH} 3.175)$ y Tenox $^{-}(\mathrm{pH} 7.150) \operatorname{con} \beta-\mathrm{CD}$

En la Figura 4.10 se muestran familias de VCs obtenidos en el sistema EPC / $160 \mu \mathrm{M}$ Tenox, $1 \mathrm{M} \mathrm{Cl}^{-}$con diferentes valores de $\mathrm{pH}$ donde predominan diferentes especies Tenox: HTenox( $z$ ) (pH 3.175) Figura 4.10a, y Tenox ${ }^{-}$(pH 7.150) Figura 4.10b. Al igual que el caso anterior se observa la formación de un pico anódico alrededor de $0.65 \mathrm{~V}$ para $\mathrm{pH}$ 3.175 y un pico de oxidación en $0.60 \mathrm{~V}$ para $\mathrm{pH} 7.150$ (en ausencia de $\beta-\mathrm{CD}$ ). Al invertir el barrido de potencial no se observa ningún pico de reducción para ambos casos. Es 
posible apreciar que al aumentar la concentración de $\beta-C D$ en ambos casos el pico de oxidación se desplaza hacia potenciales más positivos y se presenta un decremento en la corriente de pico de oxidación.
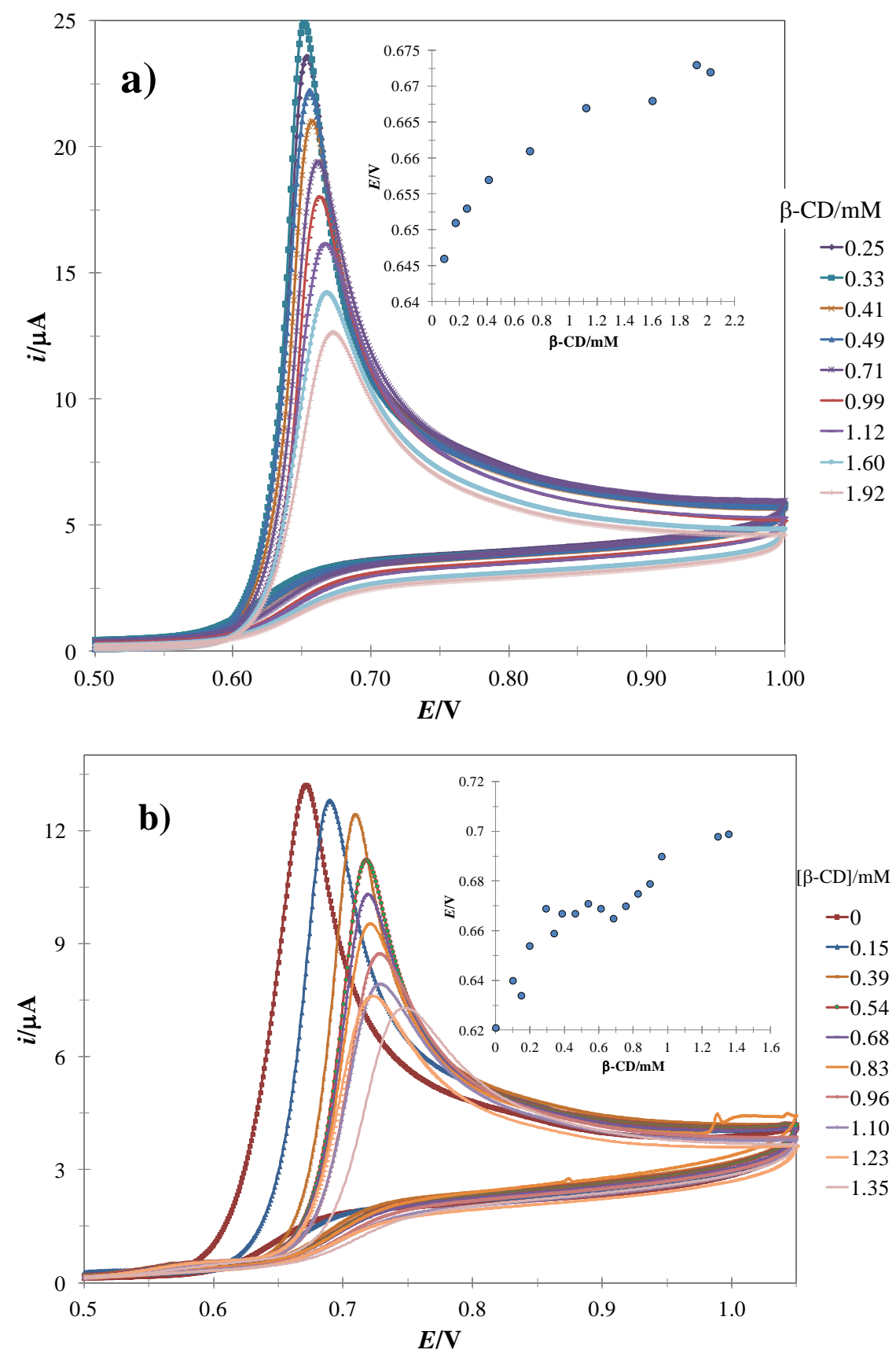

Figura 4.10. VCs obtenidos en el sistema EPC / Tenox $0.17 \mathrm{mM} \mathrm{Cl}^{-}$para diferentes concentraciones de $\beta-\mathrm{CD}$ mostradas en la figura $\mathrm{mM}$ y diferentes valores de $\mathrm{pH}$ : a) 3.175 b) 7.150. En todos los caso el barrido de potencial inició en corriente nula en dirección 
anódica a una velocidad de $0.1 \mathrm{Vs}^{-1}$. El inserto muestra la variación de la corriente de pico anódica en función de la concentración de $\beta-\mathrm{CD}$.

De igual manera siguiendo el modelo de Z.N. Gao, se obtiene la función $i_{p}^{2}=$ $f\left(\frac{i_{p(\text { Tenox })}^{2}-i_{p}^{2}}{[\beta C d]}\right)$, ver ecuación 4.13, para los dos sistemas y se obtiene la constante de inclusión respectiva en solución correspondientes a los equilibrios R2 (pH 3.175) y R3 ((pH 7.150), como se muestran en las ecuaciones 4.15 y 4.16, así como también la ecuación obtenida de la función lineal para cada sistema.

pH $3.175 i_{p}^{2}\left(A^{2}\right)=(2.29 \pm 0.15) \times 10^{-4} M * \frac{\left(i_{p(\text { Tenox })}^{2}-i_{p}^{2}\right)}{[\beta C D]}+(\mathbf{0 . 6 5} \pm \mathbf{0 . 1 7}) \times 10^{-4} A^{2}$

$R^{2}=0.990-\log K_{d}=3.640 \pm 0.063$

pH 7.150 $i_{p}^{2}\left(A^{2}\right)=(6.89 \pm 0.38) \times 10^{-4} M * \frac{\left(i_{p(\text { Tenox })}^{2}-i_{p}^{2}\right)}{[\beta C D]}+(0.16 \pm 0.04) \times 10^{-4} A^{2}$

$R^{2}=0.990-\log K_{d}=3.153 \pm 0.055$

En la Tabla 4.4 se presenta la comparación de las constantes de inclusión de Tenox en $\beta$-CD, para distintos valores de $\mathrm{pH}$, obtenidas a partir de las dos técnicas experimentales consideradas en este trabajo. Se puede notar los valores obtenidos por ambas técnicas para cada equilibrio son muy cercanos y con ambas técnicas se obtuve la misma tendencia de variación de $\mathrm{K}$ con el pH.

Tabla 4.4. Comparación de las constantes de inclusión de Tenox en $\beta$-CD, para diferentes valores de $\mathrm{pH}$, obtenidas mediante métodos espectrofotométricos y electroquímicos.

\begin{tabular}{|c|c|c|c|}
\hline \multirow[b]{2}{*}{$\mathrm{pH}$} & \multirow[b]{2}{*}{ Equilibrio } & \multicolumn{2}{|c|}{$\log \mathrm{K}_{\mathrm{f}}$} \\
\hline & & Electroquímico & Espectrofotométrico \\
\hline 0.420 & $\mathrm{H}_{2}$ Tenox $^{+}+\beta-C D \rightleftharpoons \beta-C D-H_{2}$ Tenox $^{+}$ & $3.007 \pm 0.103$ & $2.982 \pm 0.005$ \\
\hline 3.175 & $\operatorname{HTenox}(z)+\beta-C D \rightleftharpoons \beta-C D-H T \operatorname{Tenox}(z)$ & $3.640 \pm 0.063$ & $3.705 \pm 0.005$ \\
\hline 7.150 & Tenox $-\beta-C D \rightleftharpoons \beta-C D-$ Tenox - & $3.153 \pm 0.055$ & $3.285 \pm 0.017$ \\
\hline
\end{tabular}


Es importante hacer notar que a diferencia de lo reportado por Banerjee y col ${ }^{[4.11]}$, en este trabajo, utilizando dos técnicas experimentales distintas, se ha puesto en evidencia que es posible la formación de complejos de inclusión de Tenox con $\beta$-CD independientemente de la forma predominante del Tenox al variar el pH de la disolución.

\subsection{Conclusiones.}

Los resultados han demostrado la formación de complejos de inclusión para todas las especies de Tenox con $\beta$-CD por medio de dos técnicas experimentales, así mismo se han obteniendo los valores para estos complejos. 


\section{CAPÍTULO 5. COMPLEJOS DE INCLUSIÓN SUPERFICIAL.}




\section{CAPÍTULO 5. COMPLEJOS DE INCLUSIÓN SUPERFICIAL}

\subsection{Introducción.}

Considerando que en el capítulo 4 se ha demostrado la formación de complejos de las distintas especies de Tenox con la $\beta C D$ en solución, en este capítulo se estudia la formación de un complejo superficial, modificando la superficie con $\beta C D$ sobre la superficie de un electrodo de pasta de carbón (EPC).

5.2 Metodología para la determinación de los complejos de inclusión superficial.

\subsubsection{Reactivos.}

La solución de Tenox fue preparada a partir del reactivo grado A R Merck, se trabajó con una solución de $\beta-\mathrm{CD}$ (Fluka 99\%). Para ajustar el pH se utilizó NaOH (Merck 99\%) y $\mathrm{HCl} /($ Merck 37\%). Todas las soluciones fueron preparadas usando agua desionizada tipo $1(18.2 \mathrm{M} \Omega \mathrm{cm})$ libre de materia orgánica, obtenida de US Filter PURELAB Plus UV cual fue burbujeada con nitrógeno comprimido (Praxair) (1 min/mL).

\subsubsection{Equipo utilizado para los experimentos electroquímicos.}

Se utilizó una celda convencional de tres electrodos, como electrodo de trabajo se utilizó un electrodo de pasta de carbón (EPC) el cual fue preparado a partir de polvo de Grafito (Johnson Matthey $1 \mu \mathrm{m}, 99.9 \%)^{[5.1,15.2]}$. Como contra-electrodo se utilizó un alambre de platino (BAS MW-1032) y como electrodo de referencia se utilizó un electrodo $\mathrm{Ag} / \mathrm{AgCl}$ saturado (BAS MF-2052). Los experimentos electroquímicos se llevaron a cabo con un potenciostato (EPSILON-Bass-i).

El pH de las soluciones fue medido usando un potenciómetro Mettler Lab pHM240 con una resolución de 0.001 de $\mathrm{pH}$ con un electrodo combinado de vidrio de intervalo de pH 0-14 ((Mettler Toledo InLab® Expert NTC30 + DIN/RCA)). Durante la experimentación se controló la temperatura a $25^{\circ} \mathrm{C}$ y se mantuvo la solución en atmósfera inerte de nitrógeno. 
5.2.3 Construcción de un electrodo con poli- $\beta-\mathrm{CD}$.

El electrodo de trabajo se modificó utilizando una solución de $\beta-\mathrm{CD} 0.01 \mathrm{M}$ en $1 \mathrm{M}$ de ácido perclórico aplicando 30 ciclos dentro de una ventana de potencial de 0.8 a $1.3 \mathrm{~V}$ a una velocidad de barrido de potencial $0.1 \mathrm{Vs}^{-1}{ }^{[5.3]}$, por lo tanto el electrodo que ha sido modificado se denominado EPC/poli- $\beta-\mathrm{CD}$.

5.3 Respuesta del Tenoxicam con un electrodo con poli- $\beta-\mathrm{CD}$.

En la figura 5.1 se muestran los VCs obtenidos en una solución de HCL $1 \mathrm{M}$ sin Tenox utilizando el EPC/poli- $\beta-C D$. Es posible notar que la corriente capacitiva incrementa considerablemente cuando se presenta en la interface del electrodo el polímero de $\beta-C D$.

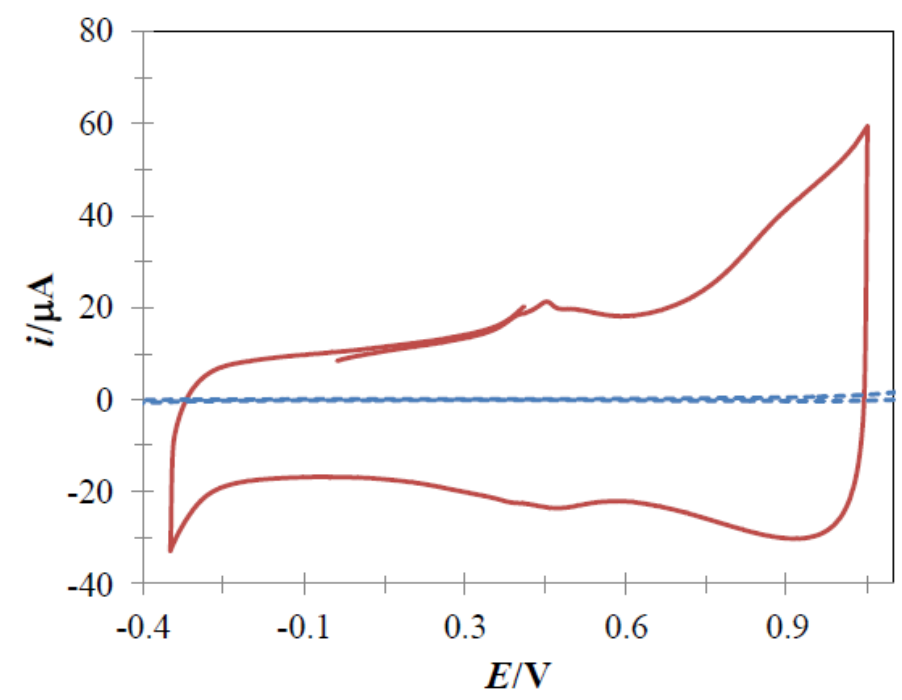

Figura 5.1. Voltamperograma cíclico para el sistema $\mathrm{HCl} 1 \mathrm{M}$ sobre un EPC (línea punteada) y sobre un EPC/poli- $\beta$-CD línea contínua. En ambos casos la velocidad de barrido de potencia es de $0.1 \mathrm{Vs}^{-1}$.

En la figura 5.2 se muestra la comparación de los voltamperogramas obtenidos para pH 3.17 (HTenox(z)) utilizando los diferentes electrodos. Note que el potencial de pico de oxidación de la especie $\operatorname{HTenox}(\mathrm{z})$ es el mismo para ambos electrodos, sin embargo cuando se utiliza el EPC/poli- $\beta-\mathrm{CD}$, la corriente de pico incrementa notoriamente, incluso cuando la concentración de $\operatorname{HTenox}(\mathrm{z})$ fue tres veces menor que en el caso del EPC sin modificar. 


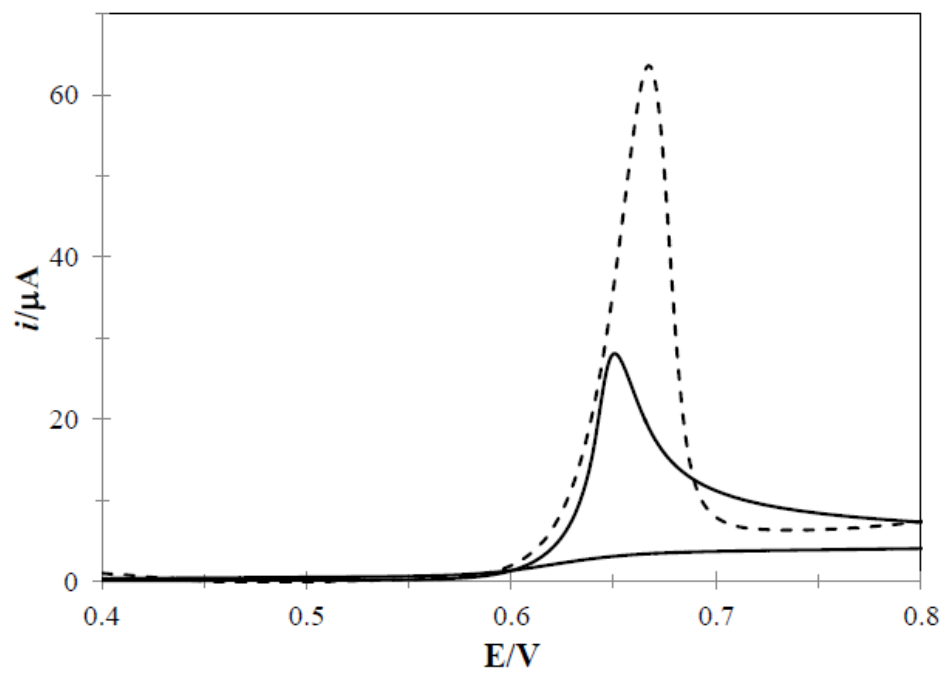

Figura 5.2. Voltamperogramas cíclicos obtenidos en dos sistemas diferentes: (línea sólida) EPC/poli- $\beta-\mathrm{CD} / \mathrm{HCl} 1 \mathrm{M}, 61 \mu \mathrm{M}$ Tenox, pH 3.17 y (línea punteada) EPC/ HCl 1 $\mathrm{M}, 203 \mu \mathrm{M}$ Tenox, en ambos casos la velocidad de barrido de potencial es de $0.1 \mathrm{Vs}^{-1}$.

En la figura 5.3 se muestra la comparación de los voltamperogramas cíclicos obtenidos para las diferentes especies de Tenox utilizando un EPC/poli- $\beta-\mathrm{CD}$ donde es posible observar que de manera similar al electrodo de EPC no modificado, mostrados en el capítulo 2, el potencial del pico de oxidación desplaza hacia potenciales más pequeñas con el aumento del $\mathrm{pH}$. 
EPC- $\beta C D$

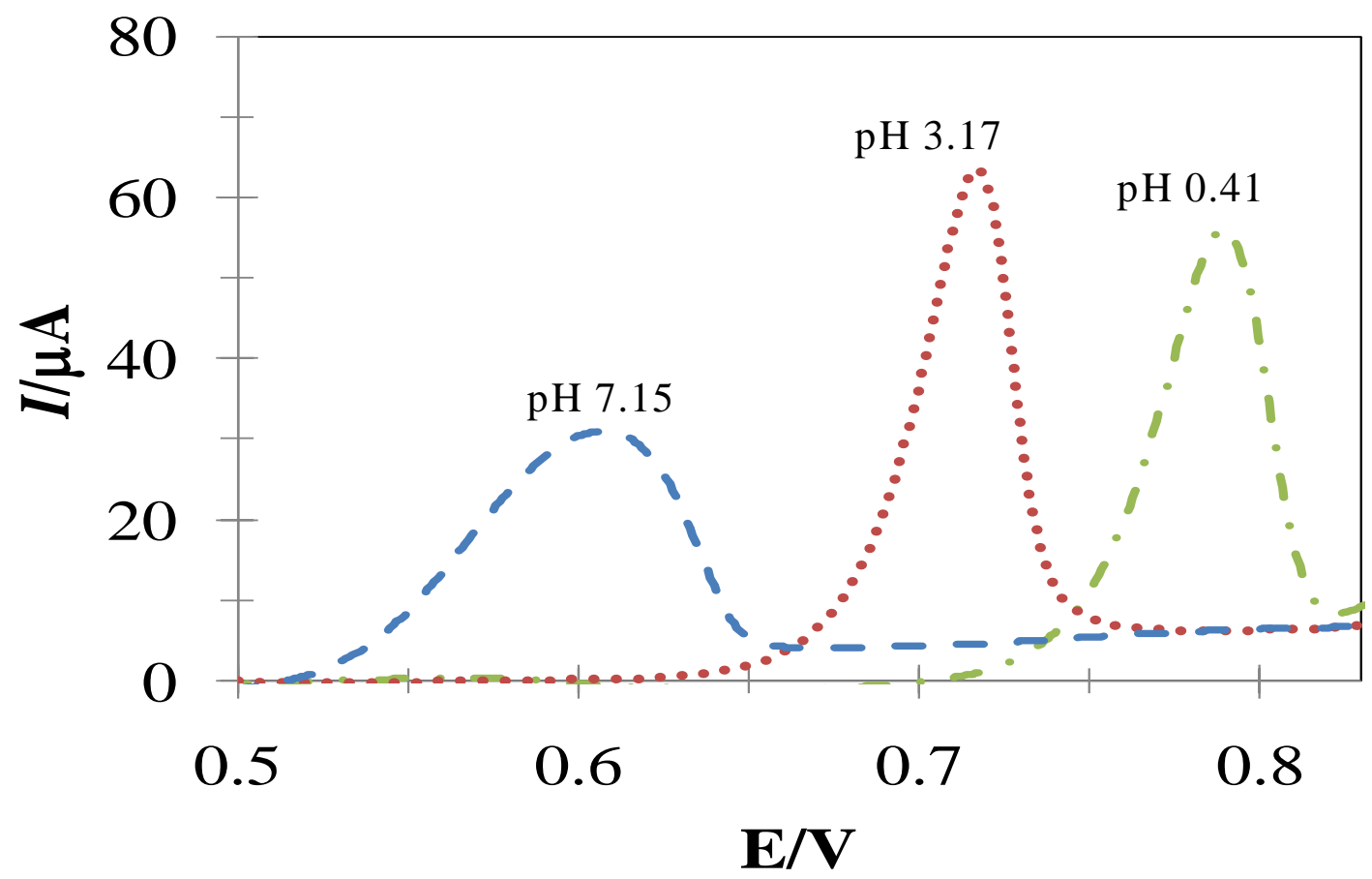

Figura 5.3. Familia de voltamperograma cíclicos obtenidos sobre un EPC/poli$\beta-\mathrm{CD} / \mathrm{HCl} 1 \mathrm{M}, 61 \mu \mathrm{M}$ de Tenox para diferentes valores de $\mathrm{pH}$ mostrados en la figura. En todos los casos se inició el barrido de potencial en corriente nula en dirección anódica, la velocidad de barrido de potencial es de $0.1 \mathrm{Vs}^{-1}$.

\subsubsection{Influencia de la velocidad de barrido de potencial.}

En la figura 5.4 se muestra ima fa,oñoa de voltamperogramas cíclicos obtenidos en el sistema EPC/poli- $\beta-\mathrm{CD} / \mathrm{HCl} 1 \mathrm{M}, 120 \mu \mathrm{M}$ Tenox a $\mathrm{pH} 0.8$, aplicando diferentes velocidades de barrido de potencial, como se muestra en el inserto, la corriente del pico depende linealmente con la velocidad de barrido, lo que sugiere fuertemente que en este caso la oxidación electroquímica del Tenox está controlada por la adsorción ${ }^{[5.3,5.4]}$. El mismo comportamiento se presenta para $\mathrm{pH} 3.17$ y 7.15 


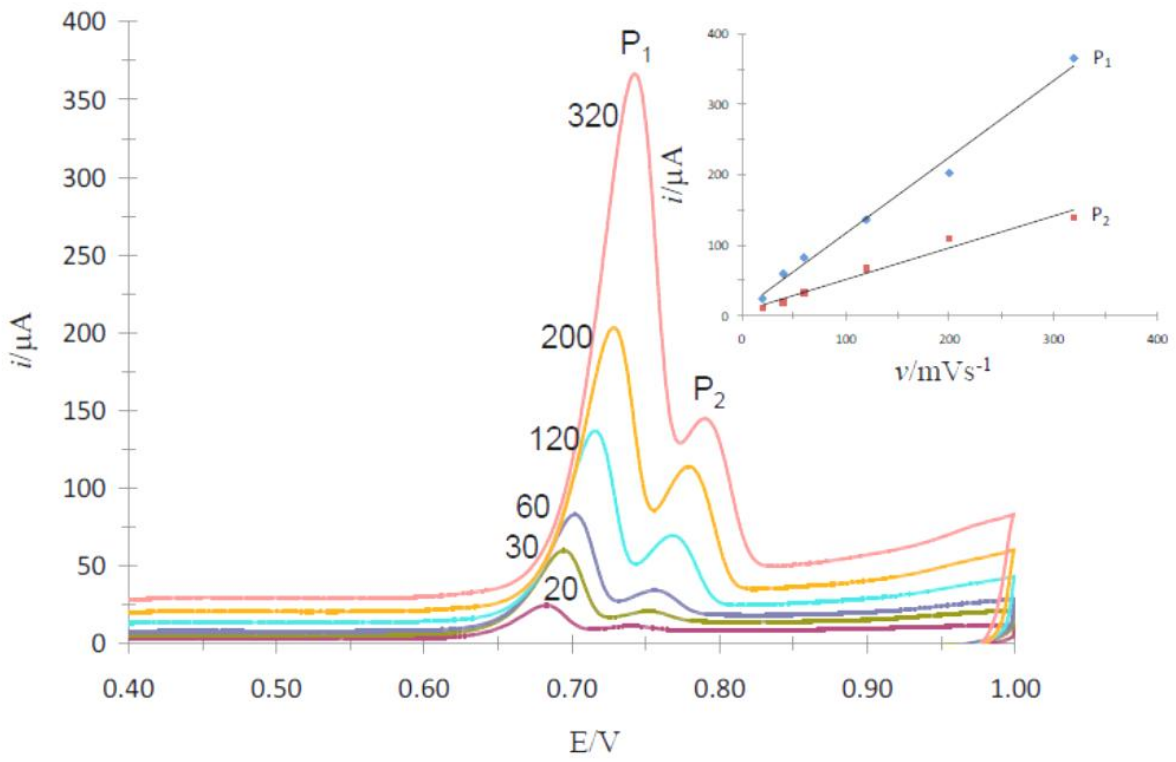

Figura 5.4. Familia de voltamperogramas cíclicos obtenidos en el sistema EPC/poli$\beta-\mathrm{CD} / \mathrm{HCl} 1 \mathrm{M}, 120 \mu \mathrm{M}$ Tenox a $\mathrm{pH} 0.8$ para diferentes velocidades de barrido de potencial indicadas en la figura, El inserto muestra la variación de corriente de pico anódico $\mathrm{P}_{1}$ y $\mathrm{P}_{2}$, como se indica en la figura, en función de la velocidad de barrido.

\subsubsection{Influencia de la velocidad de rotación del electrodo.}

La Figura 5.5 muestra que a una velocidad de rotación constante del electrodo y $\mathrm{pH}$ constante, la corriente de pico no cambia, véase el inserto en la figura. Esto mismo se observa en los otros valores de $\mathrm{pH}$ considerados en este trabajo, lo que refuerza que para todos los valores de $\mathrm{pH}$, cuando se utiliza el EPC/poli- $\beta-\mathrm{CD}$, la oxidación del Tenox es un proceso que está limitado por adsorción. 


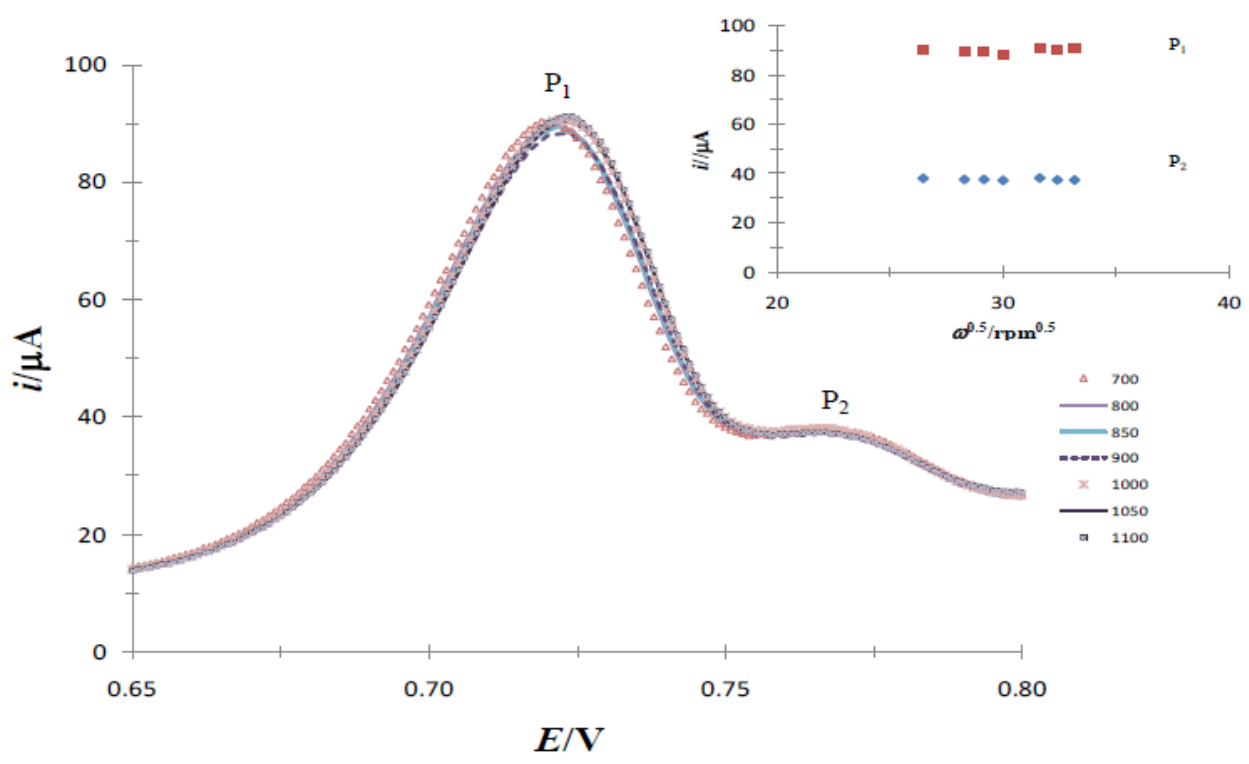

Figura 5.5. Voltamperogramas lineales obtenidos en el sistema EPC/poli- $\beta-C D /$ $\mathrm{HCl} 1 \mathrm{M}, 120 \mu \mathrm{M}$ Tenox a pH 0.8 para diferentes velocidades de rotación del electrodo las cuales se muestran en la figura. En todos los casos la velocidad de barrido de potención se impuso a $0.8 \mathrm{Vs}^{-1}$. El inserto muestra la variación de la corriente de pico dada para $\mathrm{P}_{1} \mathrm{y}_{2}$ en función de la el cuadrado de la velocidad de rotación del electrodo.

\subsubsection{Influencia de la concentración de Tenoxicam.}

En la figura 5.6 se muestra una familia de voltamperogramas para el sistema EPC/poli- $\beta-\mathrm{CD} / \mathrm{HCl} 1 \mathrm{M}, \mathrm{pH} 0.41$ aplicando una velocidad de barrido de $0.1 \mathrm{Vs}^{-1}$, se presenta la zona de potencial donde predomina el primer pico de oxidación para diferentes concentraciones de Tenox. Note que la corriente de pico del voltamperograma incrementa conforme la concentración incrementa. Lo mismo se puede observas para los pH 3.17 y 7.15 . 


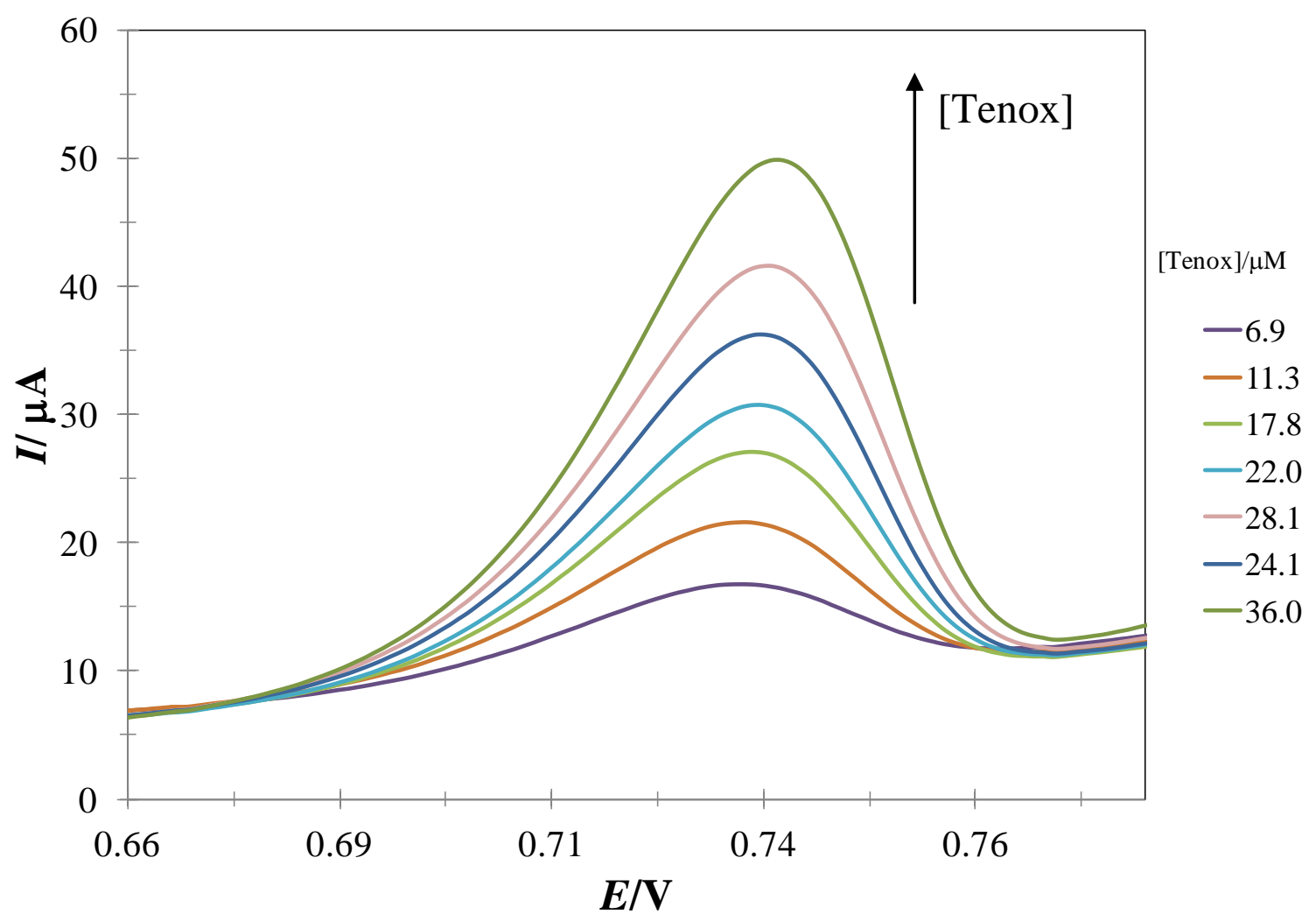

Figura 5.6. Familia de voltamperogramas obtenidos en el sistema EPC/poli- $\beta-C D /$ $\mathrm{HCl} 1 \mathrm{M}, \mathrm{pH} 0.41$ para diferentes concentraciones de Tenox de 5 a $56 \mathrm{mM}$; la flecha indica la dirección del incremento de la concentración. En todos los casos la velocidad de barrido es de $0.1 \mathrm{Vs}^{-1}$.

5.3.4 Simulación de los voltamperogramas de barrido lineal.

Considerando la evidencia presentada con respecto a que el proceso de oxidación de la molécula de Tenox sobre un EPC/poli- $\beta-C D$ y la forma de los voltamperogramas descritos por Laviron ${ }^{[5.6]}$ para una reacción superficial totalmente irreversible, se simularon los voltamperogramas de acuerdo a la ecuación (5.1) propuesta por Laviron, ver ecuación 39 en [5.6].

$$
i=\mathrm{P}_{1} \exp \left(\mathrm{P}_{2}\left(E-\mathrm{P}_{3}\right)\right) \exp \left(-\exp \left(\mathrm{P}_{2}\left(E-\mathrm{P}_{3}\right)\right)\right)
$$

Donde

$$
\begin{aligned}
& \mathrm{P}_{1}=(\mathrm{F} 2 / \mathrm{RT}){\mathrm{n} \alpha \mathrm{n}_{\alpha} \Gamma_{\mathrm{R}} v} \\
& \mathrm{P}_{2}=\alpha \mathrm{n}_{\alpha} \mathrm{F} / \mathrm{RT} \\
& \mathrm{P}_{3}=\mathrm{E}_{\mathrm{p}}
\end{aligned}
$$


Donde la $i$ es la corriente medida en función del potencial aplicado, E, n es el número de electrones transferidos durante la reacción heterogénea, $\mathrm{n}_{\alpha}$, es el valor de $\mathrm{n}$ de la etapa determinante de la velocidad, $\alpha$, es el coeficiente de transferencia, $v$ es la velocidad de barrido de potencial, R, T y F son la constante universal de los gases, la temperatura absoluta y la constante de Faraday, respectivamente, $E_{p}$ es el potencial de pico, $\Gamma_{\mathrm{R}}$ es la concentración superficial de la especie electroactiva reducida.

Como un ejemplo de la capacidad de la ecuación (5.1) para describir el proceso de oxidación de Tenox sobre el electrodo de EPC/poli- $\beta-C D$, la Figura 5.7 muestra la comparación de un voltamograma experimental lineal de barrido a pH 0.8, con uno teórica generada por la suma de dos contribuciones de adsorción tal como se describe por la ecuación (5.1). Es importante destacar que este voltamograma fue elegido porque representa uno de los casos más complicados, debido a la presencia de dos picos. Durante el proceso de ajuste no lineal todos los parámetros se les permitieron variar libremente. Hay que tener en cuenta que la descripción teórica es bastante adecuada y las incertidumbres asociadas a los parámetros de mejor ajuste, también son bastante bajos. Para el ajuste de los voltamperogramas a pH 3.17 y 7.15, las ecuaciones teóricas utilizadas incluyen sólo una contribución la cual se describe en la ecuación (5.1), ya que en ambos casos se observó un solo pico de potencial en cada uno de los voltamperogramas. 


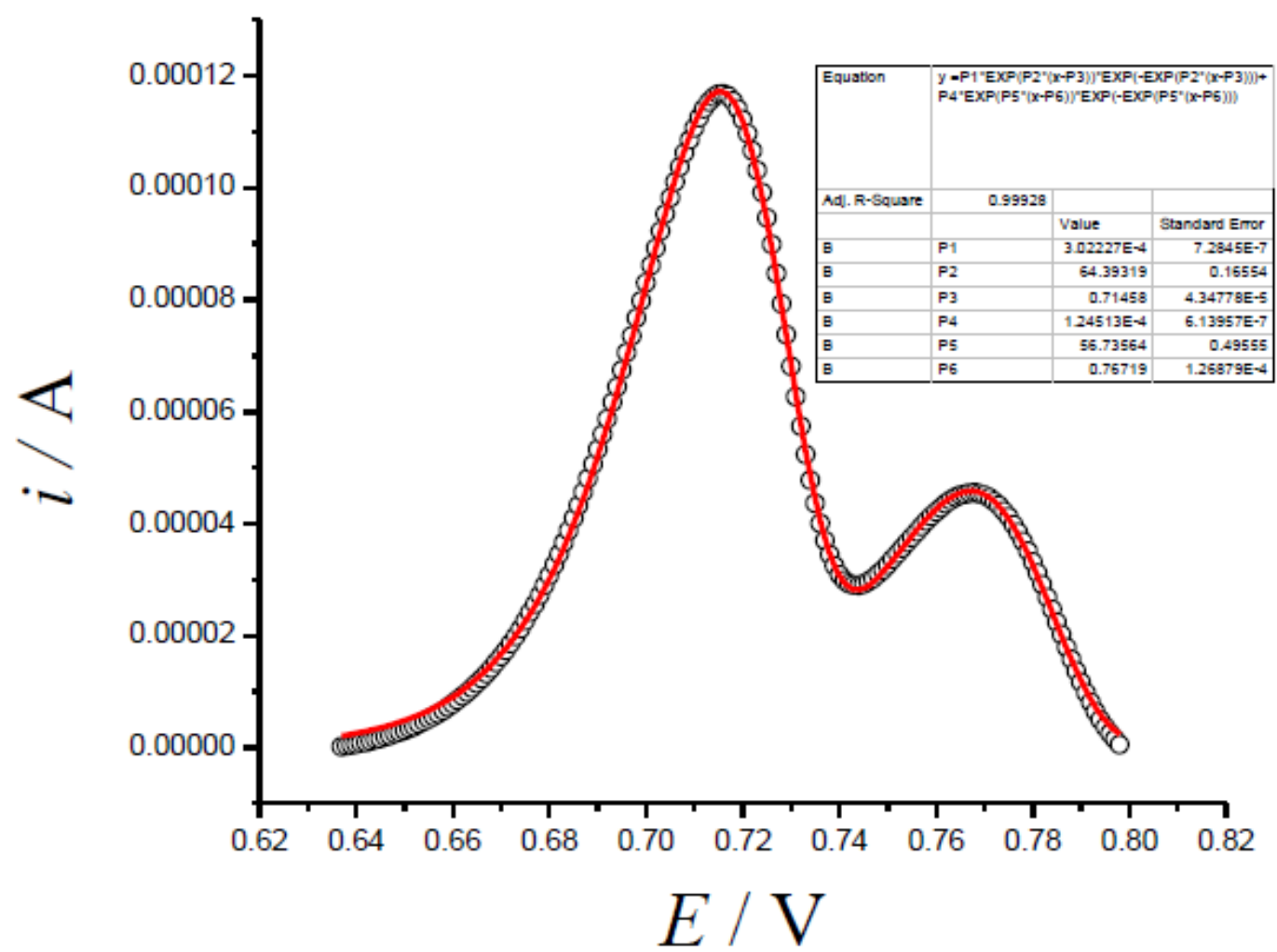

Figura 5.7. Comparación de un voltamperograma experimental (marcador) para un sistema EPC/poli- $\beta-\mathrm{CD} / \mathrm{HCl} 1 \mathrm{M}, 120 \mu \mathrm{M}$ Tenox a pH 0.8 a $0.1 \mathrm{Vs}-1$ con un voltamperograma teórico (línea continua) generado por un ajuste no lineal de dos procesos de absorción descritos en la ecuación (1). El inserto muestra la parametrización de la ecuación utilizada, los parámetros ajustados y sus incertidumbre correspondientes.

A partir de los valoes de los parámetros P1 y P2 es posible estimar la concentración de la especie electroactiva reducida en la superficie a partir de $\Gamma_{R}$ en fución de la concentración de la solución. En la tabla 5.1 es posible observar que para pH 3.17 el valor máximo de $\Gamma_{\mathrm{R}}$ se alcanzó para una concentración de Tenox de $64 \mu \mathrm{M}$. 
Tabla 5.1. Variación de la concentración de la especie reducida en la superficie a partir de $\Gamma_{\mathrm{R}}$ sobre el EPC/poli- $\beta-\mathrm{CD}$ en función de su concentración estimada a partir de los valores de los parámetros de mejor ajuste, P1 y P2, obtenidos por ajuste no lineal de la ecuación (5.1) para los voltamogramas experimentales a un $\mathrm{pH}$ de 3.17.

\begin{tabular}{|c|c|c|c|}
\hline $\mathbf{C} / \boldsymbol{\mu} \mathbf{M}$ & $\mathbf{1 0}^{\mathbf{4}} \mathbf{P}_{\mathbf{1}} / \mathbf{A}$ & $\mathbf{P}^{2} / \mathbf{V}^{-\mathbf{1}}$ & $\mathbf{1 0}^{\mathbf{1 0}} \Gamma / \mathbf{m o l c m}^{-2}$ \\
\hline 64 & 2.06 & 59 & 1.51 \\
\hline 62 & 1.98 & 58 & 1.47 \\
\hline 45 & 1.81 & 55 & 0.42 \\
\hline 35 & 1.20 & 71 & 0.72 \\
\hline 25 & 0.99 & 69 & 0.62 \\
\hline 16 & 0.45 & 67 & 0.29 \\
\hline 10 & 0.25 & 56 & 0.20 \\
\hline 7 & 0.20 & 49 & 0.18 \\
\hline
\end{tabular}

5.4 Determinación de las constantes de inclusión superficiales.

Dada la capacidad de la $\beta$-CD para formar complejos de inclusión con varios tipos de moléculas ${ }^{[5.7,5.8]}$, la adsorción del Tenox en el EPC/poli- $\beta-C D /$ puede explicarse mediante la formación de un complejo de inclusión entre las especias predominantes de la moléculas del Tenox y la $\beta$-CD que constituyen la superficie del polímero de poli- $\beta$-CD. De acuerdo con Roa Morales y otros ${ }^{[5.9]}$, la estimación de estas constantes de inclusión superficial puede llevarse a partir de los voltamperogramas experimentales obtenidos en función de la concentración del Tenox, véase la Figura 5.6, y la ecuación (5.5).

$$
\frac{i_{s a t}-i_{n}}{i_{\text {sat }}}=1-K_{\text {inc }}^{\text {sup }}[\text { Tenox }]
$$

Donde $i_{s a t}$ es la corriente de pico anódico saturada, $i_{n}$ es la corriente de pico anódico de la alicuato añadida, $K_{\text {inc }}^{\text {sup }}$ es la constante termodinámica de la formación del complejo de inclusión superficial y [Tenox] es la concentración de Tenox en solución acuosa. 
A partir de la pendiente de la gráfica $i_{\text {sat }} i_{n} / i_{\text {sat }}$ en función de [Tenox], ver figura 5.8, a los diferentes pHs considerados para el predominio de las especies de Tenox, el valor calculado de $K_{\text {inc }}^{\text {sup }}$ para cada especie se reporta la tabla 5.2.

Tabla 5.2. Variación de la constante termodinámica del complejo de inclusión superficial en función del $\mathrm{pH}$ de la solución.

\begin{tabular}{|c|c|c|c|}
\hline $\mathbf{p H}$ & $\begin{array}{c}\text { Pendiente } \mathbf{x ~ 1 0} \\
/ \mathbf{M}^{-\mathbf{1}}\end{array}$ & $\mathbf{R}^{\mathbf{2}}$ & $\log \mathbf{K}_{\text {inc }}$ sup \\
\hline 0.41 & $1.847 \pm 0.064$ & 0.995 & $4.27 \pm 0.03$ \\
\hline 3.17 & $1.824 \pm 0.040$ & 0.995 & $4.26 \pm 0.02$ \\
\hline 7.15 & $1.778 \pm 0.039$ & 0.995 & $4.25 \pm 0.02$ \\
\hline
\end{tabular}

\subsection{Conclusiones.}

Un electrodo de pasta de carbono (EPC) fue modificado con un polímero $\beta$-CD. Se encontró que la oxidación electroquímica del Tenox en el electrodo de EPC/poli- $\beta-\mathrm{CD}$ es controlada por la adsorción. Se evaluó la influencia de diferentes variables, como son, el pH solución de, velocidad de barrido de potencial, velocidad rotación del electrodo y la concentración de Tenox. Las simulaciones de los voltamperogramas experimentales se llevaron a cabo de manera adecuada teniendo en cuenta que la oxidación Tenox en el electrodo de EPC/poli- $\beta-C D$ es una reacción superficial irreversible. Se calculó la la constante de inclusión superficial formada durante la adsorción Tenox en la superficie de la membrana de poli- $\beta-\mathrm{CD}$. 


\section{CAPÍTULO 6. DETERMINACIÓN ANALÍTICA DE LA CONCENTRACIÓN DE TENOXICAM EN DISOLUCIÓN ACUOSA.}




\subsection{Introducción.}

Una vez caracterizada la molécula del Tenox, es indispensable un estudio de determinación analítica de la concentración en disoulución acuosa, ya que en el estudio de fármacos poco solubles, los límites de detección (LD) y cuantificación (LC), así como la sensibilidad del método (SM), son parámetros importantes, ya que determinan la capacidad de análisis de un método analítico, por lo que en este capítulo se estudia el comportamiento electroquímico de la concentración de Tenox en solución acuosa mediante técnicas de VC, DPV, y técnicas hidrodinámicas de disco rotatorio, así mismo se lleva a cabo la determinación mediante la modificación del electrodo de trabajo con un membrana de poli$\beta C D$.

\subsection{Metodología.}

\subsubsection{Reactivos.}

Todos los reactivos son grado analítico, Tenox (FLUKA), hidróxido de sódio (J.T. Baker) y el ácido clorhídrico (Monterrey); las soluciones fueron preparadas con agua desionizada. El desionizador utilizado fue Milli-Q (Millipore) con resistividad de 18.0 MS. El potenciómetro es $\mathrm{pH} / \mathrm{Ion}$ Anlayzer (METTLER TOLEDO). Durante el experimento las soluciones de Tenox en la celda se mantuvieron en atmósfera de nitrógeno.

\subsubsection{Equipo.}

Se utilizó una celda convencional de tres electrodos, como electrodo de trabajo se utilizó un electrodo de pasta de carbón (EPC) el cual fue preparado a partir de polvo de Grafito (Johnson Matthey $1 \mu \mathrm{m}, 99.9 \%$ ). Un potenciostato BAS 100 B /W y el disco rotatorio BAS-EDR-2 se utiliza para estudios electroquímicos con una celda convencional de tres electrodos: electrodo de platino (contraelectrodo BAS-MW-1033), Ag/AgCl (electrodo de referencia BAS-MF-2079) y electrodo de pasta de carbón (electrodo de trabajo BAS-MF-2065 RDE punta para electrodos de pasta $3 \mathrm{~mm}$ diámetro). 
6.3 Cuantificación Tenox sobre un electrodo de pasta de carbón.

\subsubsection{Estudio de cuantificación utilizando un EDR.}

En la figura 6.1 se presentan los voltamperogramas lineales de disco rotatorio para distintas adiciones de Tenox de 0 a $88 \mu \mathrm{M}$ a pH 0.405 para el estudio de determinación de Tenox, en los cuales se puede observar que la corriente límite aumenta con la concentración.

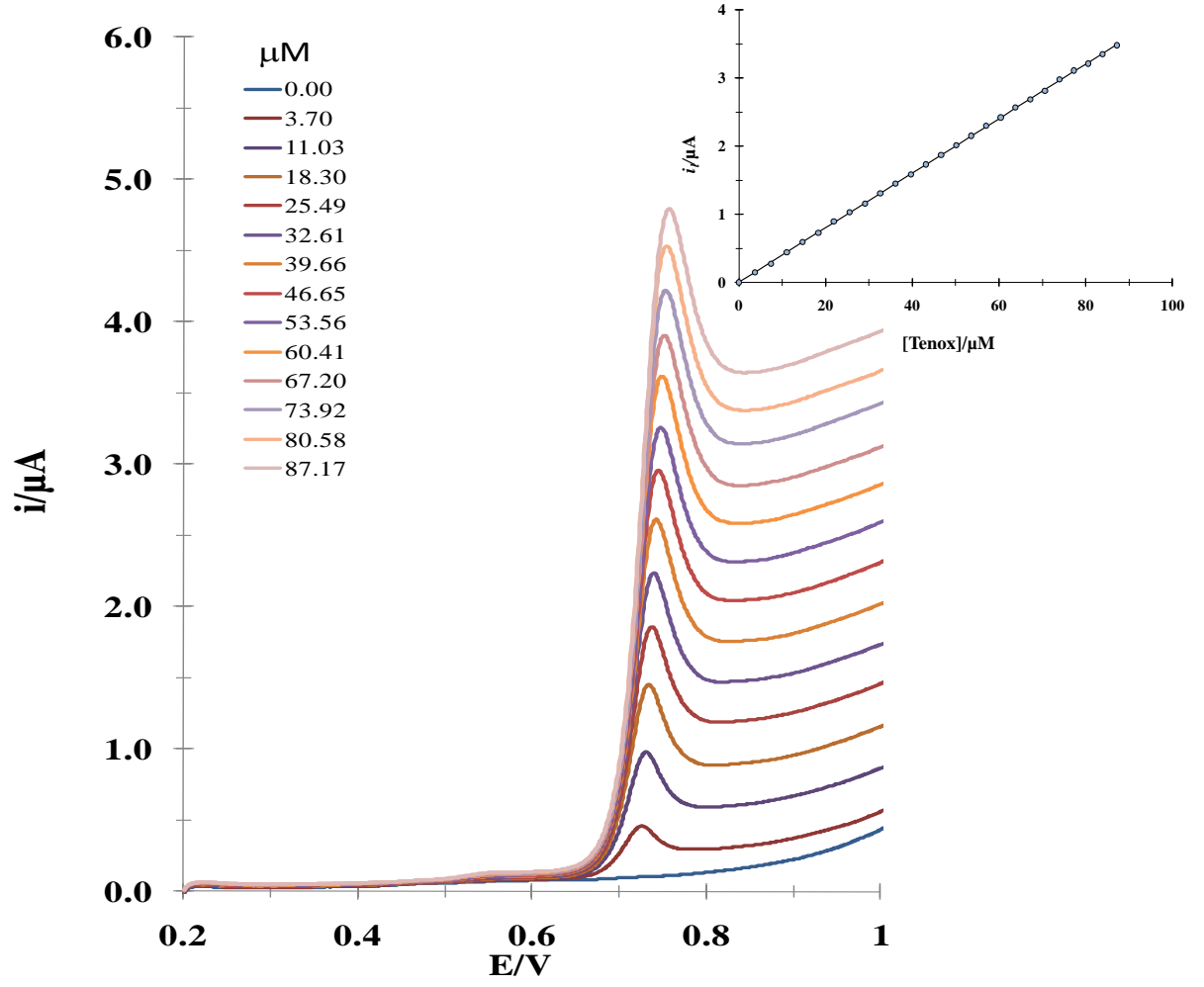

Figura 6.1. Voltamperogramas EDR a velocidad de barrido de potencial de $0.1 \mathrm{~V} \mathrm{~s}^{-1}$ y $\omega=240 \mathrm{rpm}$ de [Tenox] $0-88 \mu \mathrm{M}$ en pH 0.405. En el inserto se presenta la gráfica $\mathrm{I}=\mathrm{f}[\mathrm{Tenox}]$ a $0.9 \mathrm{~V}$ 
En el inserto de la figura 6.1 se aprecia la curva de calibración, para esta se impuso un potencial de $0.9 \mathrm{~V}$ y se observa una tendencia lineal de la corriente en función de la concentración.

La ecuación de la recta del inserto de la figura 6.1 corresponde a $i_{L}=(0.04003 \pm 0.00008) \mu \mathrm{A} \mu \mathrm{M}^{-1} \mathrm{x}+(0.002 \pm 0.004) \mu \mathrm{A}$, con una correlación de $\mathrm{R}^{2}$ 0.9999 .

El mismo estudio de concentraciones se realizó para diferentes velocidades de barrido de potencial $\left(0.01-0.1 \mathrm{~V} \mathrm{~s}^{-1}\right)$ para una velocidad de rotación constante y se obtuvo el LD y LC de cada uno, esto valores se observan en la tabla 6.1.

Tabla 6.1. Cálculo del límite de detección y cuantificación para Tenox a distinta velocidades de barrido a pH 0.405 en el sistema EDR/ $1 \mathrm{M} \mathrm{HCl}$

\begin{tabular}{|c|l|l|l|}
\hline Velocidad de barrido $\left(\mathbf{m V s}^{-\mathbf{1}}\right)$ & $\mathbf{L D}(\boldsymbol{\mu M})$ & $\mathbf{L C}(\boldsymbol{M} \mathbf{M})$ & Sensibilidad $\left(\boldsymbol{\mu} \mathbf{A} \boldsymbol{\mu} \mathbf{M}^{\mathbf{- 1}}\right)$ \\
\hline 10 & $3.32 \pm 1.18$ & $11.08 \pm 1.16$ & $0.01656 \pm 0.00014$ \\
\hline 25 & $3.67 \pm 1.3$ & $12.23 \pm 1.28$ & $0.03137 \pm 0.00029$ \\
\hline 50 & $3.67 \pm 1.4$ & $12.23 \pm 1.36$ & $0.03137 \pm 0.00029$ \\
\hline 75 & $1.36 \pm 0.48$ & $4.52 \pm 0.48$ & $0.03989 \pm 0.00013$ \\
\hline 100 & $0.79 \pm 0.28$ & $2.64 \pm 0.28$ & $0.04003 \pm 0.00008$ \\
\hline
\end{tabular}

Es posible apreciar en la tabla 6.1 que conforme incrementa la velocidad de barrido de potencial se presenta una mejora en los valores del límite de detección, de cuantificación y sensibilidad.

6.3.2 Estudio de cuantificación por voltamperometría cíclica.

En la figura 6.2 se puede observar una familia de voltamperogramas típicos obtenidos para Tenox a distintas adiciones de Tenox de 0 a $88 \mu \mathrm{M}$ a pH 0.405 , donde se observa que la corriente de pico anódico crece debido al incremento de concentración de Tenox. 


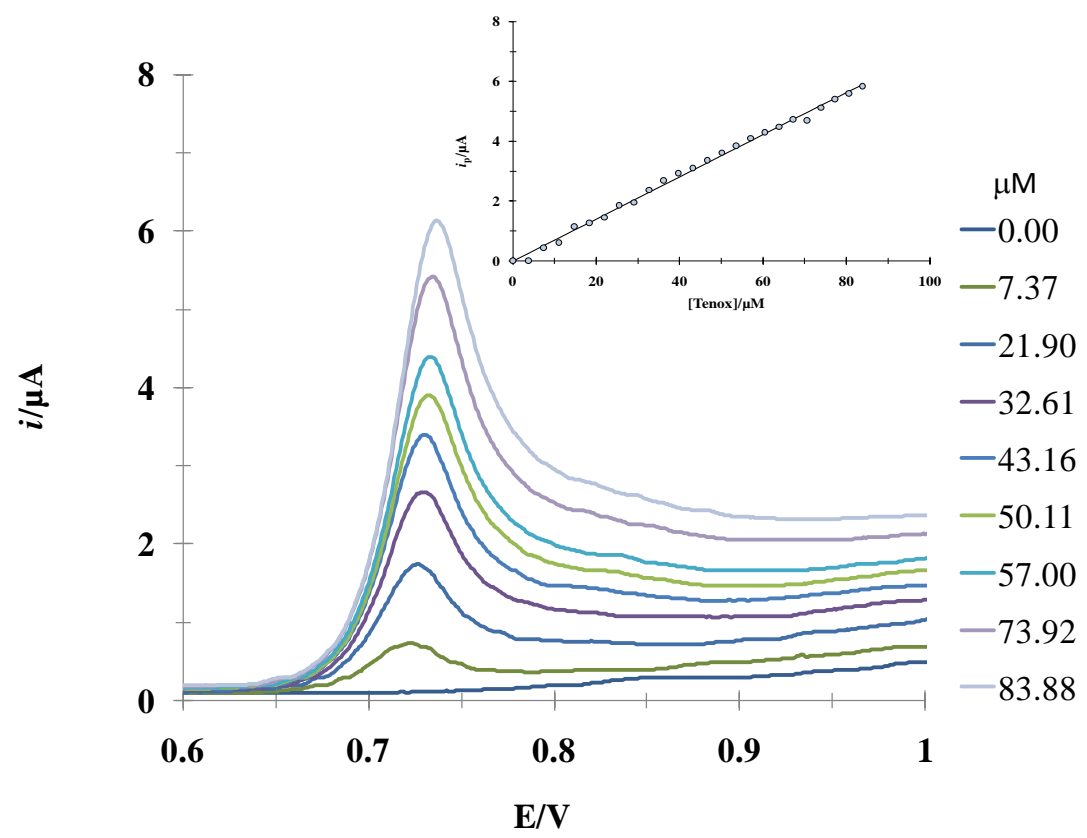

Figura 6.2. Voltamperogramas VC a velocidad de barrido de potencial de $100 \mathrm{mV} \mathrm{s}^{-1}$ de [Tenox] 0-88 $\mu \mathrm{M}$ en $\mathrm{pH}$ 0.405. En el inserto se presenta la gráfica $\mathrm{I}=\mathrm{f}([$ Tenox $]) \mathrm{pH} 0.405$.

La ecuación de la recta que se presenta en el inserto de la figura 6.2 corresponde a $\mathrm{Ip}=(0.0705 \pm 0.0009) \mu \mathrm{A} \mu \mathrm{M}^{-1} \mathrm{x}+(0.015 \pm 0.044) \mu \mathrm{A}$ con una correlación de $\mathrm{R}^{2}=0.9962$.

En la tabla 6.2 se presenta el cálculo de los límites de detección y cuantificación

Tabla 6.2. Cálculo del límite de detección y cuantificación para Tenox a distinta velocidades de barrido a pH 0.405 en el sistema EPC/ $1 \mathrm{M} \mathrm{HCl}$.

\begin{tabular}{|c|c|c|c|}
\hline Velocidad $\left(\mathrm{mVs}^{-1}\right)$ & $\mathbf{L D} \mu \mathbf{M}$ & $\mathbf{L C} \mu \mathrm{M}$ & Sensibilidad $\mu \mathrm{A} \mu \mathrm{M}^{-1}$ \\
\hline 100 & $4.57 \pm 1.74$ & $15.22 \pm 1.74$ & $0.0562 \pm 0.001$ \\
\hline 75 & $4.09 \pm 1.53$ & $13.62 \pm 1.53$ & $0.0413 \pm 0.0005$ \\
\hline 50 & $3.79 \pm 1.45$ & $12.64 \pm 1.45$ & $0.032 \pm 0.0005$ \\
\hline 25 & $4.74 \pm 1.75$ & $15.8 \pm 1.75$ & $0.0257 \pm 0.0003$ \\
\hline 10 & $4.03 \pm 1.53$ & $13.43 \pm 1.47$ & $0.0152 \pm 0.0002$ \\
\hline
\end{tabular}

En la tabla 6.2 se aprecia que para una velocidad de $50 \mathrm{mVs}^{-1}$ se tiene el mejor valor para el límite de detección y cuantificación, sin embargo la mejor 
sensibilidad se presenta para una velocidad de $10 \mathrm{mVs}^{-1}$. Para llevar a cabo una determinación por medio de voltamperometría cíclica dependerá del parámetro analítico que se desee mejorar.

6.3.3 Estudio de cuantificación por voltamperometría diferencial de pulso.

La cuantificación del Tenox mediante VDP, se realizó haciendo adiciones de Tenox de 0 a $21.5 \mu \mathrm{M}$ a para el sistema de EPC / $\mathrm{HCl} 0.1 \mathrm{M}$, ( $\mathrm{pH}=0.405)$ con una amplitud de pulso de $0.025 \mathrm{~V}$ a 200 milisegundos para velocidades de 10, 20, 25, 30 y $50 \mathrm{mVs}^{-1}$. En la figura 6.3 se observa un voltamperograma típico de VDP a $20 \mathrm{mVs}^{-1}$.

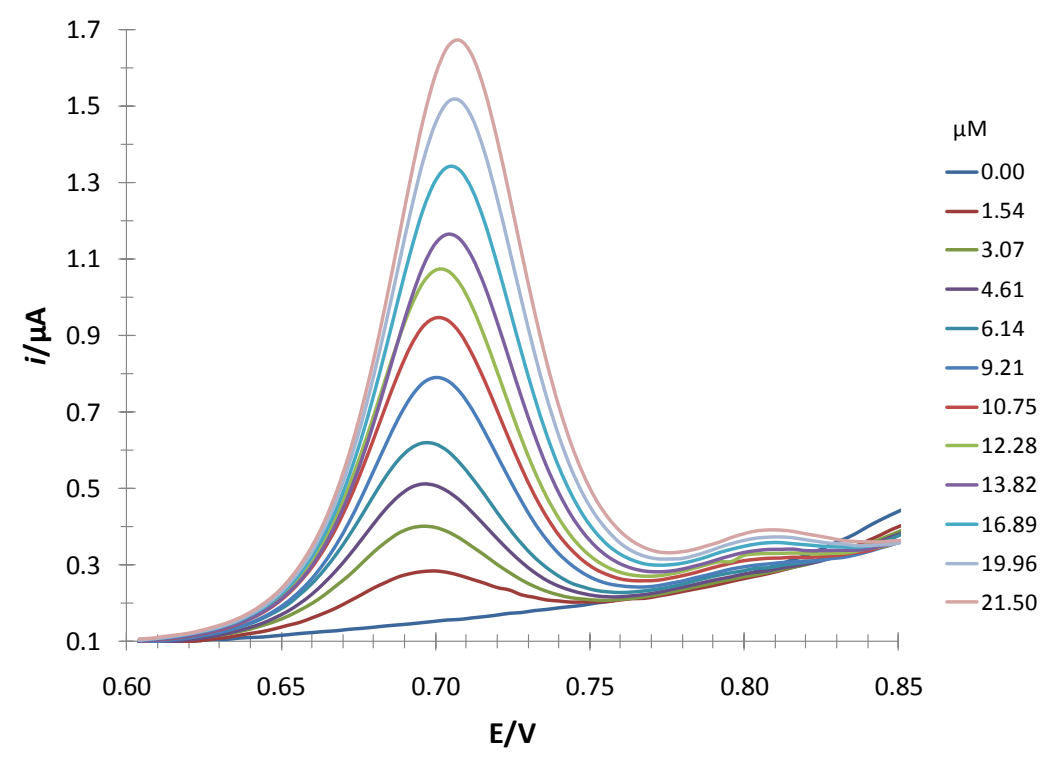

Figura 6.3. VDP para el sistema de EPC / $\mathrm{HCl} 0.1 \mathrm{M}$, Tenox 0-21.5 $\mu \mathrm{M}(\mathrm{pH}=0.405)$ para una velocidad de barrido de $20 \mathrm{mV} \mathrm{s}^{-1}$.

Se obtuvieron las curvas de calibración para diferentes velocidades de barrido de potencial observándose una tendencia lineal de la corriente de pico en función de la concentración de Tenox.

En la tabla 6.3 se presenta la ecuación de las rectas obtenidas para diferentes velocidades de barrido de potencial y se aprecia un incremento de la pendiente con forme incrementa la velocidad de barrido potencial. 
Tabla 6.3. Ecuación para las distintas velocidades de barrido de potencia obtenidas del sistema VDP sobre EPC/HCl 0.1 M, Tenox 0-21.5 mM (pH=0.405)

\begin{tabular}{|c|c|c|}
\hline VDP & Ecuación de la recta & $\mathbf{R 2}$ \\
\hline $10 \mathrm{mVs}^{-1}$ & $\mathrm{Ip}=(0.0438 \pm 0.0004) \mu \mathrm{A} \mu \mathrm{M}-1 \mathrm{x}+(0.0156 \pm 0.0040) \mu \mathrm{A}$ & 0.999 \\
\hline $20 \mathrm{mVs}^{-1}$ & $\mathrm{Ip}=(0.0700 \pm 0.0011) \mu \mathrm{A} \mu \mathrm{M}-1 \mathrm{x}+(0.0273 \pm 0.0118) \mu \mathrm{A}$ & 0.997 \\
\hline $25 \mathrm{mVs}^{-1}$ & $\mathrm{Ip}=(0.0745 \pm 0.00091) \mu \mathrm{A} \mu \mathrm{M}-1 \mathrm{x}+(0.0223 \pm 0.0091) \mu \mathrm{A}$ & 0.998 \\
\hline $30 \mathrm{mVs}^{-1}$ & $\mathrm{Ip}=(0.1000 \pm 0.0039) \mu \mathrm{A} \mu \mathrm{M}-1 \mathrm{x}+(0.0238 \pm 0.0364) \mu \mathrm{A}$ & 0.984 \\
\hline $50 \mathrm{mVs}^{-1}$ & $\mathrm{Ip}=(0.0863 \pm 0.0017) \mu \mathrm{A} \mu \mathrm{M}-1 \mathrm{x}-(0.048 \pm 0.016) \mu \mathrm{A}$ & 0.984 \\
\hline
\end{tabular}

Los límites de detección y cuantificación obtenidos a partir de las curvas presentadas en la tabla 6.3 se presentan en la tabla 6.4.

Tabla 6.4. Límites de detección y cuantificación para las distintas velocidades de barrido de potencial asociados a las curvas de la tabla 4.

\begin{tabular}{|c|c|c|}
\hline VDP & Límite de detección $(\mu \mathrm{M})$ & $\begin{array}{c}\text { Límite de } \\
\text { cuantificación }(\mu \mathrm{M})\end{array}$ \\
\hline $\mathbf{1 0} \mathbf{~ W V s}^{-1}$ & $0.51 \pm 0.19$ & $1.70 \pm 0.19$ \\
\hline $\mathbf{2 0} \mathbf{~ W V s}^{-1}$ & $0.96 \pm 0.35$ & $3.21 \pm 0.34$ \\
\hline $\mathbf{2 5}{\mathbf{~} V \mathbf{s}^{-1}}^{-1}$ & $0.68 \pm 0.25$ & $2.25 \pm 0.25$ \\
\hline $\mathbf{3 0} \mathbf{~ V V s}^{-1}$ & $1.98 \pm 0.72$ & $6.59 \pm 0.69$ \\
\hline $\mathbf{5 0}{\mathbf{~} V \mathbf{s}^{-1}}^{-1}$ & $0.96 \pm 0.36$ & $3.19 \pm 0.35$ \\
\hline
\end{tabular}

Se aprecia en la tabla 6.4 que el mejor límite de detección y cuantificación se presentan para una velocidad de barrido de potencial a $30 \mathrm{mVs}^{-1}$ sin embargo en la tabla6.3 para $30 \mathrm{mVs}^{-1}$ se puede observar que no presenta la mejor correlación, por lo que para llevar a cabo la cuantificación de Tenox la velocidad de barrido de potencial adecuada es a $25 \mathrm{mVs}^{-1}$ presentando esta misma el menor límite de detección y de cuantificación. 
En la figura 6.4 se aprecia las gráficas de velocidad de barrido de potencial en función de la concentración de Tenox donde se hace la comparación de los límites de detección, cuantificación y sensibilidad respectivamente, para cada técnica electroquímica utilizada.
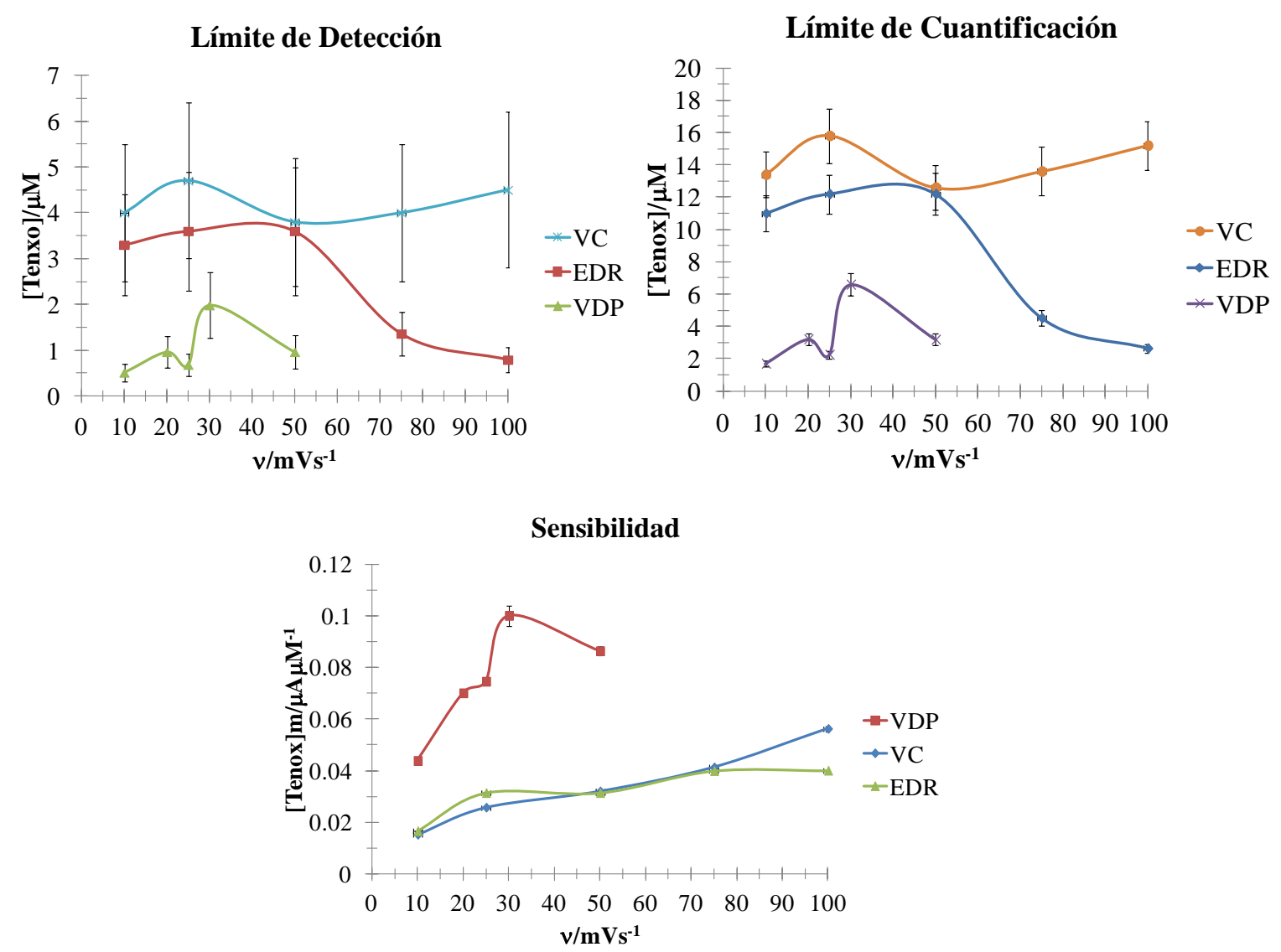

Figura 6.4. Comparación de límite de detección para las diferentes técnicas electroquímicas mostradas en la figura para el sistema EPC / $\mathrm{HCl} 0.1 \mathrm{M}$, Tenox 0-21.5 $\mu \mathrm{M}$ $(\mathrm{pH}=0.405)$

Es posible apreciar en la figura 6.4 de manera más fácil lo que indican las tablas 6.1, 6.2 y 6.4, que los mejores límites de detección y cuantificación con el menor error asociado así como la mejor sensibilidad se presentan para la técnica de VDP. 
6.3.4 Estudio de cuantificación por voltamperometría cíclica para un electrodo de pasta de cabón modificado con un polímero de $\beta$-CD.

Dado que en electroquímica la técnica más común para caracterizar y llevar a cabo un estudio de determinación analítica es la voltamperometría cíclica, se llevo a cabo un estudio de cuantificación por medio de esta técnia utilizando un electrodo modificado con un polímero de $\beta$-CD con el fin de mejorar los parámetros analíticos.

En la figura 6.5 se puede observar una familia de voltamperogramas típicos obtenidos para Tenox a distintas adiciones de Tenox de 0 a $55 \mu \mathrm{M}$ a $\mathrm{pH} 0.405$, donde se observa que la corriente de pico anódico crece debido al incremento de concentración de Tenox.

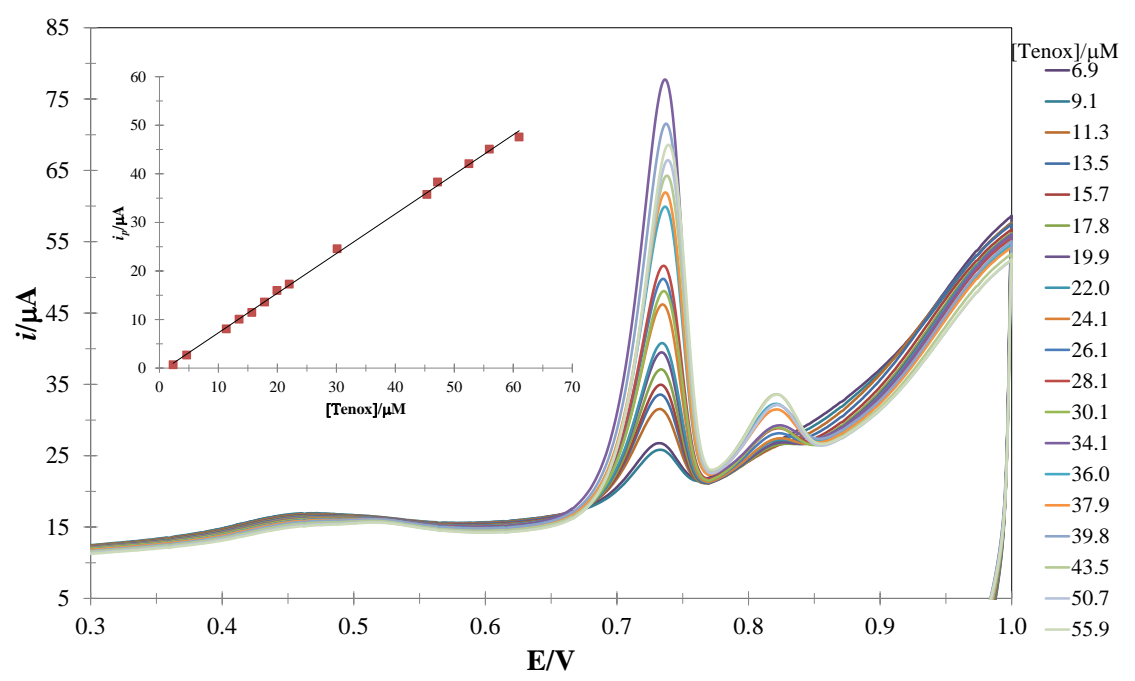

Figura 6.5. Voltamperogramas VC para un EPC modificado con un polímero de $\beta$-CD a velocidad de barrido de potencial de $100 \mathrm{mV} \mathrm{s}^{-1}$ de [Tenox] 0-55 $\mu \mathrm{M}$ en $\mathrm{pH} 0.405$. En el inserto se presenta la gráfica $\mathrm{I}=\mathrm{f}([$ Tenox $]) \mathrm{pH} 0.405$.

En la tabla 6.5 se presentan los límites de detección y cuantificación así como la sensibilidad obtenidos a partir de la curva de calibración descrita en el inserto de la figura 6.5, se puede apreciar que comparado con los parámetros obtenidos sobre un EPC sin modificar. 
Tabla 6.5. Comparación del límite de detección, cuantificación y sensibilidad para Tenox pH 0.405 en el sistema EPC- $\beta-\mathrm{CD} / 1 \mathrm{M} \mathrm{HCl}$ con el sistema $\mathrm{EPC} / 1 \mathrm{M} \mathrm{HCl}$

\begin{tabular}{|l|l|l|c|}
\hline \multicolumn{1}{|c|}{ Sistema } & $\mathbf{L D}(\boldsymbol{\mu M})$ & $\mathbf{L C}(\boldsymbol{\mu M})$ & $\operatorname{Sensibilidad}\left(\boldsymbol{\mu} \mathbf{A} \boldsymbol{\mu} \mathbf{M}^{\mathbf{1}}\right)$ \\
\hline EPC- $\boldsymbol{\beta}$-CD/ 1 M HCl & $0.86 \pm 0.28$ & $4.93 \pm 0.69$ & $0.815 \pm 0.008$ \\
\hline EPC/ 1 M HCl & $4.57 \pm 1.74$ & $15.22 \pm 1.74$ & $0.0562 \pm 0.001$ \\
\hline
\end{tabular}

En la tabla 6.5 se aprecia que los valores de límite de detección, cuantificación y sensibilidad presentan una mejora incluso aún cuando la concentración de Tenox.

\subsection{Conclusiones.}

Se obtuvieron los mejores parámetros electroquímicos para la determinación analítica de Tenox utilizando un electrodo de pasta de carbono para diferentes técnicas electroquímicas (voltamperometría cíclica, voltamperometría lineal con electrodo de disco rotatorio y voltamperometría diferencial de pulso). Se llevo a cabo un estudio de cuantificación para un electrodo modificado con un polímero de $\beta$-CD obteniendo una mejora en los limites de cuantificación, detección y sensiblidad cuanto se trabaja con este último. 


\section{CONCLUSIONES}

En este trabajo se llevó a cabo el estudio de un antiinflamatorio no esteroidal (AINE), el Tenoxicam, utilizando espectroscopía UV-Vis, métodos electroquímicos y métodos de química cuántica.

En primer lugar, se determinaron las zonas de predominio de diferentes especies: $\mathrm{H}_{2} \operatorname{Tenox}^{+}$, HTenox(z), HTenox ${ }^{-}$en función del $\mathrm{pH}$ de la solución, así como dos valores de $\mathrm{pKa}$ correspondientes a las transformaciones entre dichas especies.

Por medio de métodos electroquímicos (voltamperometría cíclica, voltamperometría lineal con electrodo de disco rotatorio, voltamperometría diferencial de pulso y cronoamperometría) y computacionales (DFT), se determinó que la oxidación de Tenox sigue un mecanismo electroquímico-químico en el que se pierden dos electrones. La molécula original se fragmenta y se forman principalmente dos productos no electroactivos, el 2-metil-2H-tieno [2,3-e][1,2] tiazina-3,4-diona 1,1 -dióxido y el ácido piridina-2ilcarbámico. Así, la reacción de oxidación es irreversible, lo cual se observa claramente en las gráficas de voltamperometría cíclica.

A fin de mejorar la estabilidad y el aumento de la solubilidad, se propuso complejar la molécula de Tenox con $\beta$-ciclodextrina. Utilizando las técnicas experimentales de espectroscopía UV-Vis y métodos electroquímicos, se demostró que la formación del complejo de inclusión en solución acuosa se lleva a cabo independientemente de la especie predominante de Tenox. 
Se determinaron los parámetros analíticos para la determinación de Tenox en solución acuosa sobre un electrodo de pasta de carbono, pudiéndose detectar una concentración mínima de $15.22 \pm 1.74 \mu \mathrm{M}$. También se propuso una técnica para mejorar el límite de detección, el límite de cuantificación, la linealidad y la sensibilidad en la detección de Tenox en agua. Para ello, se diseñó un electrodo de pasta de carbono modificado con un polímero de $\beta$-Ciclodextrina, Se determinó que, a diferencia de lo que ocurre en el electrodo sin modificar en el que la oxidación está limitada por la difusión, en el caso del electrodo modificado la oxidación electroquímica está controlada por la adsorción de Tenox sobre el polímero. Se evaluó la influencia de diferentes variables (pH, velocidad de barrido de potencial, velocidad de rotación del electrodo y concentración de Tenox), y se obtuvieron las constantes de inclusión superficial. El electrodo modificado permite detectar concentraciones de Tenox de $4.93 \pm 0.69 \mu \mathrm{M}$, lo que representa una concentración tres veces menor que con el eletrodo sin modificar. 


\section{BIBLIOGRAFÍA}

\section{INTRODUCCIÓN.}

[I.1] A. Bello, Vademécum Farmacológico-Terapéutico, p. 684, .Ed. Andrés Bello, (1991)

[I.2] J. Flórez, Farmacología humana, p. 396-397, 4ª Edición. Ed. Masson, (2003)

[I.3] B. Santos Ramos y M. D. Guerrero Aznar, Administración de medicamentos Teoría y Práctica, p. 47, Ediciones Díaz Santos, S.A., (1994)

[I.4] Szeijtli, J. Chem Rev. 98 (1998), 1743-1753.

[I.5] M I. Nicolás Vázquez, E. Marín Chiñas, F. M. Castro Martínez, R. Miranda

Ruvalcaba, Algunos Conceptos Básicos de la Química Computacional, p 11, Comité editorial UNAM (2006).

[I.6] D. Rodríguez-Barrientos, A. Rojas-Hernández, A. Gutiérrez, R. Moya-Hernández, R. Gómez-Balderas, M. T. Ramírez-Silva; Talanta 80 (2009) 754-762

[I.7] M. N. Hamers and D. Roos, Oxidative stress in human neutrophilic granylocytes: host defense and self-defense. In "Oxidative Stress" (H. Sies, ed.), pp. 351-381. Academic Press, New York, 1985.

[I.8] R. A. Clark, Adv. inflammation Res. Extracellular effects of the myeloperoxidasehydrogen peroxide-system. 5 (1983) 107-146.

[I.9] P.S. Wong and J. Travis, Biochem. Biophys. Res. Commun. 96 (1980) 1449-1454.

[I.10] N. R. Matheson, P. 5. Wong, M. Schuyler, J. Travis, Biochemistry 20 (1981) 331336.

[I.11] M. Wasil, B. Halliwell, C. P. Moorhouse, D. C. S. Hutchison, and H. Baum, Biochem. Pharmacol. 36 (1987) 3847-3850.

[I.12] S. Ichihara, H. Tomisawa, H. Fukazawa, M. Tateishi, R. Joly, and R. Heintz, Biochem. Pharmacol. 35 (1986) 3935-3939.

[I.13] S. Ichihara, H. Tomisawa. H. Fukazawa, and M. Tateishi, Biochem. Pharmacol. Involvement of leukocyte peroxidases in the metabolism of Tenox. 34 (1985) 1337-1338.

[I.14] P. Van Antwerpen, J. Dubois, M. Gelbcke, J. Neve, Free Radic. Res. 38 (2004) 51258.

[I.15] S. Ichihara, H. Tomisawa, H. Fukasawa, M. Tateishi, A. Joly, A. Heintz, Drug Metab. Dispos. Oxidation of Tenox by leukocyte and $\mathrm{H}_{2} \mathrm{O}_{2}$ produces novel products. 17 (1989) 463. 
[I.16] N. A. El-Maali, J.-C. Vire, G. J. Patriarche, M.A. Ghandour, G.D. Christian, Anal. Sci., 6, 245-250, (1990).

[I.17] N. A. El-Maali and R. M. Hassan, Bioelectrochem. Bioener., 24, 155-163, (1990).

[I.18] N. Özaltin, Anal. Chim. Acta, 406, 183-189, (2000).

[I.19] F. Tsopelas, M. Ochsenkühn-Petropoulou, N. Zikos, E. Spyropoulou, I. Andreadou, A. Tsantili-Kakoulidou, Electrochemical study of some non-steroidal anti-inflammatory drugs: solvent effect and antioxidant activity. J. Solid State Electrochem. 15 (2011) 10991108.

[I.20] M. B. Gholivand, N. Karimian, Materials Science and Engineering C 31 (2011) $1844-1851$.

[I.21] A. Abbaspour, R. Mirzajani J. Pharm. Biomed. Anal. 44 (2007) 41-48

[I.22] Z. Aigner, A. Kezsmarki, M. Kata, C. Novak, I. Erös, Investigation of Tenox and $\gamma$ Cyclodextrin Binary and Ternary Complexix J. Incl. Phen. Macro. Chem. 42, (2002) 227233.

[I.23] L.M. Suta, L. Vlaia, V. Vlaia, N. G. Hadaruga, D. I. Hadrauga, C. Mircioiu, Water content of oxicam/cyclodextrin nanoparticles, J. Agroaliment. Proc. Technol. 17(4) (2011), 419-427.

[I.24] M. Palomar-Pardavé, G. Alarcón-Angeles, M.T. Ramírez-Silva, M. Romero- Romo, A. Rojas-Hernández, S. Corona-Avendaño, Electrochemical and spec-trophotometric determination of the formation constants of the ascorbic acid- $\beta$-cyclodextrin and dopamine$\beta$-cyclodextrin inclusion complexes J. Incl. Phen. Macro. Chem. 69 (2011), 91-99.

[I.25] S. Senel, O. CAkoglu, M. Sumnu, D. Duchene, A. Hincal, Preparation and Investigation of the Tenox/ $\beta$-Cyclodextrin Complex J. Incl. Phen. Molec. Reco. Chem. 14 (1992), 171-179.

[I.26] R. Banerjee, H. Chakraborty, M. Sarkar, Host-Guest Complexation of Oxicam NSAIDs with $\beta$-Cyclodextrin. Biopolymers. 75 (2004), 355-365. 


\section{CAPÍTULO 1.}

[1.1] Po-Chang Chiang, Kimberly A. Foster, Marie C. Whittle, Ching-Chiang Su, and Denise K. Pretzer., Journal of Liquid Chromatography \& Related Technologiesw, 29, 2291-2301, (2006).

[1.2] D. Rodríguez-Barrientos, A. Rojas-Hernández, A. Gutiérrez, R. Moya-Hernández, R. Gómez-Balderas, M. T. Ramírez-Silva; Talanta 80, 754-762, (2009).

[1.3] D. J. Leggett, Computational Methods for Determination of Formation Constant, p.160, Ed. Plenum Press New York and London (1985).

[1.4] Y. Zhao, N. E. Schultz, D. G. Truhlar, J. Chem. Theory Comput., 2006, 2, 364.

[1.5] A. V. Marenich, C. J. Cramer, D. G. Truhlar, J. Phys. Chem. B, 2009, 113, 6378.

[1.6] Gaussian 09, Revision A.02, M. J. Frisch, G. W. Trucks, H. B. Schlegel, G. E. Scuseria, M. A. Robb, J. R. Cheeseman, G. Scalmani, V. Barone, B. Mennucci, G. A. Petersson, H. Nakatsuji, M. Caricato, X. Li, H. P. Hratchian, A. F. Izmaylov, J. Bloino, G. Zheng, J. L. Sonnenberg, M. Hada, M. Ehara, K. Toyota, R. Fukuda, J. Hasegawa, M. Ishida, T. Nakajima, Y. Honda, O. Kitao, H. Nakai, T. Vreven, J. A. Montgomery, Jr., J. E. Peralta, F. Ogliaro, M. Bearpark, J. J. Heyd, E. Brothers, K. N. Kudin, V. N. Staroverov, R. Kobayashi, J. Normand, K. Raghavachari, A. Rendell, J. C. Burant, S. S. Iyengar, J. Tomasi, M. Cossi, N. Rega, J. M. Millam, M. Klene, J. E. Knox, J. B. Cross, V. Bakken, C. Adamo, J. Jaramillo, R. Gomperts, R. E. Stratmann, O. Yazyev, A. J. Austin, R. Cammi, C. Pomelli, J. W. Ochterski, R. L. Martin, K. Morokuma, V. G. Zakrzewski, G. A. Voth, P. Salvador, J. J. Dannenberg, S. Dapprich, A. D. Daniels, O. Farkas, J. B. Foresman, J. V. Ortiz, J. Cioslowski, and D. J. Fox, Gaussian, Inc., Wallingford CT, 2009. 
CAPÍTULO 2.

[2.1] M.T. Ramírez, M.E. Palomar, I. González, A. Rojas-Hernández, Electroanalysis 7

(1995) 184-188

[2.2] R. Martínez, M.T. Ramírez, I. González, Electroanalysis 10, 5 (1998) 336-342.

[2.3] D.K. Gosser Jr., Cyclic Voltammetry Simulation and Analysis of Reaction Mechanisms, VCH, Weinheim, 1993

[2.4] A. J. Bard and L. R Faulkner, Electrochemical Methods and Applications, Wiley Interscience, New York (2000).

[2.5] E. P. Parry, R. A. Osteryoung, Evaluation of Analytical Pulse Polarography, Anal. Chem., 37 (1965) 1634-1637.

[2.6] C.M.A. Brett, A.M.O. Brett, 1993. Electrochemistry Principles, Methods and Applications. Oxford, UK: Oxford University Press.

[2.7] J.A. Cobos-Murcia, L. Galicia, A. Rojas-Hernándeza, M.T. Ramírez-Silva, R. Álvarez-Bustamante, M. Romero-Romo, G. Rosquete-Pina, M. Palomar-Pardavé, Polymer, 46 (2005) 9053-9063

[2.8] A. Einstein, on brownian motion in fluids, Ann. Phys. (Leipzig), 17 (1905) 549-560.

[2.9] G. G. Stokes, Mathematical and Physical Papers (Cambridge University Press, Cambridge, 1903), Vol. 3 (esp. Sect. IV).

[2.10] Frisch, M. J.; Trucks, G. W.; Schlegel, H. B.; Scuseria, G. E.; Robb, M. A.; Cheeseman, J. R.; Montgomery, Jr., J. A.; Vreven, T.; Kudin, K. N.; Burant, J. C.; Millam, J. M.; Iyengar, S. S.; Tomasi, J.; Barone, V.; Mennucci, B.; Cossi, M.; Scalmani, G.; Rega, N.; Petersson, G. A.; Nakatsuji, H.; Hada, M.; Ehara, M.; Toyota, K.; Fukuda, R.; Hasegawa, J.; Ishida, M.; Nakajima, T.; Honda, Y.; Kitao, O.; Nakai, H.; Klene, M.; Li, X.; Knox, J. E.; Hratchian, H. P.; Cross, J. B.; Bakken, V.; Adamo, C.; Jaramillo, J.; Gomperts, R.; Stratmann, R. E.; Yazyev, O.; Austin, A. J.; Cammi, R.; Pomelli, C.; Ochterski, J. W.; Ayala, P. Y.; Morokuma, K.; Voth, G. A.; Salvador, P.; Dannenberg, J. J.; Zakrzewski, V. G.; Dapprich, S.; Daniels, A. D.; Strain, M. C.; Farkas, O.; Malick, D. K.; Rabuck, A. D.; Raghavachari, K.; Foresman, J. B.; Ortiz, J. V.; Cui, Q.; Baboul, A. G.; Clifford, S.; Cioslowski, J.; Stefanov, B. B.; Liu, G.; Liashenko, A.; Piskorz, P.; Komaromi, I.; Martin, R. L.; Fox, D. J.; Keith, T.; Al-Laham, M. A.; Peng, C. Y.; Nanayakkara, A.; Challacombe, 
M.; Gill, P. M. W.; Johnson, B.; Chen, W.; Wong, M. W.; Gonzalez, C.; Pople, J. A.; Gaussian 03, Revision E.01, Gaussian, Inc., Wallingford CT, 2004.

\section{CAPÍTULO 3.}

[3.1] Y. Zhao, N. E. Schultz, D. G. Truhlar, J. Chem. Theory Comput., 2006, 2, 364.

[3.2] A. V. Marenich, C. J. Cramer, D. G. Truhlar, J. Phys. Chem. B, 2009, 113, 6378.

[3.3] Gaussian 09, Revision A.02, M. J. Frisch, G. W. Trucks, H. B. Schlegel, G. E. Scuseria, M. A. Robb, J. R. Cheeseman, G. Scalmani, V. Barone, B. Mennucci, G. A. Petersson, H. Nakatsuji, M. Caricato, X. Li, H. P. Hratchian, A. F. Izmaylov, J. Bloino, G. Zheng, J. L. Sonnenberg, M. Hada, M. Ehara, K. Toyota, R. Fukuda, J. Hasegawa, M. Ishida, T. Nakajima, Y. Honda, O. Kitao, H. Nakai, T. Vreven, J. A. Montgomery, Jr., J. E. Peralta, F. Ogliaro, M. Bearpark, J. J. Heyd, E. Brothers, K. N. Kudin, V. N. Staroverov, R. Kobayashi, J. Normand, K. Raghavachari, A. Rendell, J. C. Burant, S. S. Iyengar, J. Tomasi, M. Cossi, N. Rega, J. M. Millam, M. Klene, J. E. Knox, J. B. Cross, V. Bakken, C. Adamo, J. Jaramillo, R. Gomperts, R. E. Stratmann, O. Yazyev, A. J. Austin, R. Cammi, C. Pomelli, J. W. Ochterski, R. L. Martin, K. Morokuma, V. G. Zakrzewski, G. A. Voth, P. Salvador, J. J. Dannenberg, S. Dapprich, A. D. Daniels, O. Farkas, J. B. Foresman, J. V. Ortiz, J. Cioslowski, and D. J. Fox, Gaussian, Inc., Wallingford CT, 2009.

[3.4] F. Tsopelas, M. Ochsenkühn-Petropoulou, N. Zikos, E. Spyropoulou, I. Andreadou, A. Tsantili-Kakoulidou, Electrochemical study of some non-steroidal anti-inflammatory drugs: solvent effect and antioxidant activity. J. Solid State Electrochem. 15 (2011) 10991108.

[3.5] S. Ichihara, H. Tomisawa. H. Fukazawa, and M. Tateishi, Biochem. Pharmacol. Involvement of leukocyte peroxidases in the metabolism of Tenox. 34 (1985) 1337-1338.

[3.6] P. Van Antwerpen, J. Dubois, M. Gelbcke, J. Neve, Free Radic. Res. 38 (2004) 51258.

[3.7] S. Ichihara, H. Tomisawa, H. Fukasawa, M. Tateishi, A. Joly, A. Heintz, Drug Metab. Dispos. Oxidation of Tenox by leukocyte and $\mathrm{H}_{2} \mathrm{O}_{2}$ produces novel products. 17 (1989) 463.) 
[3.8] Camaioni, D.M. y Schwerdtfeger, C.A., Comment on (2005) Accuarate Experimental Values for the Free Energies of Hydration of $\mathrm{H}^{+}, \mathrm{OH}^{-}$y $\mathrm{H}_{3} \mathrm{O}^{+}$. J. Phys. Chem. A 109, 10795-10797

\section{CAPÍTULO 4.}

[4.1] M. Palomar-Pardavé, G. Alarcón-Angeles, M.T. Ramírez-Silva, M. RomeroRomo, A. Rojas-Hernández, S. Corona-Avendaño, Electrochemical and spectrophotometric determination of the formation constants of the ascorbic acid- $\beta$ cyclodextrin and dopamine- $\beta$-cyclodextrin inclusion complexes J. Incl. Phen. Macro. Chem. 69 (2011), 91-99.

[4.2] M.T. Ramírez, M.E. Palomar, I. González, A. Rojas-Hernández, Electroanalysis7 (1995) 184-188.

[4.2] R. Martínez, M.T. Ramírez, I. González, Electroanalysis 10, 5 (1998) 336-342.

[4.3] D.J. Legget, Computational Methods for Determination of Formation Constants. Ed. Plenum Press New York, 1985.

[4.4] Puigdomenech, I. MEDUSA (Making Equilibrium Diagrams Using Sophisticated Algorithms), http://www.kemi.kth.se/medusa/

[4.5] A. Rojas, Ma.T. Ramírez, J. G. Ibáñez, I. González, Relationship of multidimensional predominance-zone diagrams with multiconditional constants for complexation equilibria Anal. Chim. Acta 246 (1991) 435-442.

[4.6] A. Rojas-Hernández, M.T. Ramírez, J.G. Ibáñez, I. González, Construction of Multicomponent Pourbaix Diagrams Using Generalized Species. J. Electrochem. Soc. 138 (1991) 365-371.

[4.7] A. Rojas-Hernández, M.T. Ramírez, I. González, J.G. Ibáñez, Multi-dimensional predominance-zone diagrams for polynuclear chemical species Anal. Chim. Acta. 259 (1992) 95-104.

[4.8] A. Rojas-Hernández, M.T. Ramírez, I. González, Equilibria among condensed phases and a multi-component solution using the concept of generalized species: Part I. Systems with mixed complexes Anal. Chim. Acta 27 (1993) 321-333. 
[4.9] A. Rojas-Hernández, M.T. Ramírez, I. González, Equilibria among condensed phases and a multi-component solution using the concept of generalized species: Part II. Systems with polynuclear species Anal. Chim. Acta 278 (1993) 335-347.

[4.10] Z.-N. Gao, X.-LWen, H.-L. Li; Study of the Inclusion Complexes of Catecholamines with $\square$-Cyclodextrin by Cyclic Voltammetry Polish J.Chem, 76 (2002), 1001-1007.

[4.11] R. Banerjee, H. Chakraborty, M. Sarkar, Host-Guest Complexation of Oxicam NSAIDs with $\beta$-Cyclodextrin. Biopolymers. 75 (2004), 355-365.

\section{CAPÍTULO 5.}

[5.1] A. Rojas, I. González, Anal. Chim. Acta, Relationship of multidimensional predominance-zone diagrams with multiconditional constants for complexation equilibria, 246 (1991) 435-442.

[5.2] A. Rojas-Hernández, M. T. Ramírez, J. G. Ibáñez and I. González, Construction of Multicomponent Pourbaix Diagrams Using Generalized Species, J. Electrochem. Soc., 138 (1991) 365-371.

[5.3] S. Corona-Avendaño, M.T. Ramírez-Silva, M. Romero-Romo, A. RojasHernández,M. Palomar-Pardavé, Influence of the $\mathrm{HClO}_{4}$ concentration on the $\beta-\mathrm{CD}$ electropolimerization over a carbon paste electrode and on dopamine's electrochemical response, Electrochim. Acta 89 (2013) 854- 860.

[5.4] D.K. Gosser Jr., Cyclic Voltammetry Simulation and Analysis of Reaction Mechanisms, VCH, Weinheim, 1993

[5.5] A.J. Bard, L.R. Faulkner, Electrochemical Methods and Applications, Wiley Interscience, New York, 2000.

[5.6] E. Laviron, Adsorption, autoinhibition and autocatalysis in polarography and in linear potential sweep voltammetry, J. Electroanal. Chem. 52 (1974) 355-393. [5.7] A. Fragoso, E. Almirall, R. Cao, L. Echegoyen, R. González-Jonte, A supramolecular approach to the selective detection of dopamine in the presence of ascorbate. Chem. Commun. 19 (2004) 2230-2231.

[5.8] M. Palomar-Pardavé, G. Alarcón-Angeles, M.T. Ramírez-Silva, M. Romero-Romo, A. Rojas-Hernández, S. Corona-Avendaño, Electrochemical and spectrophotometric determination of the formation constants of the ascorbic acid- $\beta$-cyclodextrin and 
dopamine- $\beta$-cyclodextrin inclusion complexes. J. Incl Phenom. Macrocycl. Chem., 69, (2011) 92-99.

[5.9] Gabriela Roa-Morales, Laura Galicia, Alberto Rojas-Hernández, María Teresa

Ramírez-Silva, Electrochemical study on the selective formation of [Pb(cyclodextrin)2+]

surface inclusion complexes at the carbon paste electrode/ $\mathrm{ClO}_{4-} \mathrm{M}^{\mathrm{M}}$ interphase, Electrochim. Acta 50 (2005) 1925-1930. 